\title{
Finite Automorphism Groups of Complex Tori of Dimension Two
}

\author{
By
}

Akira FUJIKI**

\section{Introduction and Summary of Resulis}

Denote by $(T, G)$ a pair consisting of a complex torus $T$ and a finite subgroup of Aut $T$, where Aut $T$ is the automorphism group of $T$ as a complex Lie group. Then the purpose of this paper is to give, in case of dimension 2, a complete classification of such pairs up to isomorphisms. Here "up to isomorphisms" means that we consider $G$ modulo conjugacy in Aut $T$. Since the body of the paper is technical and lengthy, we should like here to give a rather detailed summary of the content of this paper.

First, note that the corresponding result is well-known in the case of dimension 1. Namely, Aut $T$ is trivial, that is, $=\{ \pm 1\}$ except the cases where $T$ is isomorphic to either of the elliptic curves $E_{i}$ and $\mathbb{E}_{\rho}$; and in the latter case Aut $T$ is a cyclic group of order 4 and 6 respectively. (Here, and in what follows, we shall use freely the notations listed after this introduction.) However, in dimension 2, the result already becomes considerably complicated; for example new features appear such as the existence of "moduli", of automorphisms of infinite order, and of non-algebraic complex tori etc.

To our knowledge, the results obtained so far in this case are as follows.

a) Bolza in 1888 classified all the pairs $(G, g)$ consisting of a hyperelliptic curve $C$ of genus 2 and an automorphism $g$ of $C$ (cf. [13] for a modern presentation). Each of such automorphisms then induces an automorphism of the associated Jacobian $\mathscr{J}(C)$ of $C$ preserv

Communicated by M. Kashiwara, January 30, 1987.

*Department of Mathematics, Faculty of General Education, Kyoto University, Kyoto 606, Japan. 
ing the canonical polarization, and vice versa. Later, using this result, Enriques-Severi [5] classified all the pairs $(T, G)$ as above, under the assumption that $T=J(G)$ for some $C$ as above, $G$ preserves the canoical principal polarization of $T$ and that every element of $G$ preserves any holomorphic 2-form on $T$. In our terminology the last condition is expressed as $(T, G)$ being special (cf. below). Actually they considered also the case where $G$ is a finite automorphism group of $T$ as a complex manifold (not necessarily as a Lie group) containing no translations.

b) Gottschling [8] [9] studied the action of the Siegel modular group $\operatorname{Sp}(2, \mathbb{Z})$ on the Siegel upper half space of degree 2, and determined the stabilizer group at each point, considered modulo certain equivalence, which was later made precise by Ueno [25] to the exact $\operatorname{Sp}(2, \mathbb{Z})$-equivalence. This result is considered as determining all the pairs $(T, G)$ for which $T$ is a principally polarized abelian surface (not necessarily Jacobian) and $G$ is the group of all automorphisms of $T$ preserving the polarization.

c) Tokunaga-Yoshida [24] classified all the discrete affine automorphism group $\Delta$ of $\mathbb{C}^{2}$ which is generated by "reflections" and for which the quotient $\mathbb{C}^{2} / \Delta$ is compact. This can again be interpreted as the classification of the pairs $(T, G)$ with $G$ generated by reflections.

d) Yoshihara [27] and Fujiki [6] determined certain special cases when $G$ is cyclic. In fact our motivation for this paper comes from [6], where we used such pairs $(T, G)$ to obtain examples of certain symplectic $V$-manifolds.

In this paper we shall give exact correspondences between the above mentioned results and ours (cf, 3.4 and 4.6). For automorphisms of abelian surfaces in positive characteristic we refer the reader to the recent article by Katsura [12].

Now we turn to the detailed description of the results and methods of this paper. Let $(T, G)$ be a pair with $\operatorname{dim} T=2$ as above. Then by considering the induced actions of $G$ on the tangent space of $T$ at the origin and on the first homology group $\Lambda=H_{1}(T, \mathbb{Z})$, we get two faithful representations $f: G \rightarrow \mathrm{GL}_{2}(C)$ and $\nu: G \rightarrow \mathrm{GL}_{4}(\mathbb{Z})$ satisfying the compatibility condition $\nu_{c} \sim f \oplus \vec{f}$. The conjugacy classes of the images of $G$ by $f$ and $\nu$ in $\mathrm{GL}_{2}(\mathbb{C})$ and $\mathrm{GL}_{4}(\mathbb{Z})$ respectively then depend only on the isomorphism class of the pair $(T, G)$. We call 
them the complex and the rational representation types of $(T, G)$, respectively. They are basic invariants of the pair $(T, G)$ which are invariant under deformations. Indeed, a "moduli space" $\mathscr{M}$ (simply the set of isomorphism classes of the pairs here) of fixed complex and rational representation types turns out to have a priori a natural structure of a disjoint union of quotients $\Gamma \backslash W$ of a homogeneous complex manifold $W$ by a certain discrete group $\Gamma$ (cf。2.1, b))。 (Note however that $\Gamma$ may not act on $W$ properly discontinuously, and hence in general $\mathscr{M}$ has no analytic structure.)

Therefore, it would be natural, first to classify all the possible compatible complex and rational representation types, next to determine the structure of moduli spaces $\mathscr{M}$ with fixed complex and rational representation types as (a union of) quotients $\Gamma \backslash W$ as above -as a final outcome of our classification each $\mathscr{M}$ actually turns out to be connected (Theorem 4.7) - and finally to get description of the pairs belonging to each $\mathscr{M}$ as explicitly as possible.

First, in Section 2 we classify all the possible rational representation types of pairs (Proposition 2.14) and determine the corresponding moduli spaces (Proposition 2.19), except for certain most complicated cases as explained below. However, instead of dealing directly with subgroups $H$ of $\mathrm{GL}_{4}(\mathbb{Z})$ (as was done in certain simplest cases in [6]), we rather classify more manageable object, that is, certain quadruples $(A, \mathfrak{D}, H, M)$ which (determines and) is determined by $H$; namely, here $A$ is the $Q$-algebra generated by the elements of $H$ in $M_{4}(Q), a=A \cap M_{4}(\mathbb{Z})$ considered as an order of $A$ containing $H$ in its unit group $\mathfrak{D}^{\times}$, and finally $M=\mathscr{Z}^{4}$ considered as an $\mathfrak{0}$-module. The exceptional cases mentioned above then correspond to the cases where $A \cong M_{2}(K)$ for some imaginary quadratic field $K$. Since a rational representation type determines uniquely a compatible complex representation type except for a few simple cases (cf. 2.8) this already takes care of the first two steps other than the exceptional cases. Each member of the resulting moduli spaces will then be made explicit in the subsequent sections. On the other hand, the exceptional cases will be classified directly (cfo below). In these cases $T$ is necessarily a singular abelian surface (cf. 5.1 for the definition) and it turns out that such exceptional pairs $(T, G)$ are finite in number。

From the viewpoint of complex representation $f: G \rightarrow G_{2}(\mathbb{C})$ the 
subgroup $G_{0}:=f^{-1}\left(\mathrm{SL}_{2}(\mathbb{C})\right)$ is important. $G$ then normalizes $G_{0}$ and the quotient $G / G_{0}$ is cyclic of order $\leqq 6$ (cf. Lemma 3.2). In general we call a pair $(T, G)$ special if $G=G_{0}$, i. e., $f(G) \cong \mathrm{SL}_{2}(C)$. The problem is then reduced to the following two steps; to study special paris $(T, G)$ and then to study the normalizer of $G$ in Aut $T$.

Now in Section 3 we classify special pairs. We see easily that the essential cases are (cf. Lemma 3.3): Case $A: G$ is cyclic of order 4 or 6, and Case $B: G$ is isomorphic to either of the groups $\mathfrak{Q}, \mathfrak{I}$ or D (cf. Notations). Moreover, in each case the complex and the rational representation types are unique. First, in Case $\mathrm{A}$ the moduli space is shown to be a quotient of the total space of the holomorphic tangent bundle of the complex projective line $\mathbb{P}^{1}$ modulo certain discrete group whose action is not properly discontinuous (Proposition 3.5). Also the period matrix description of each member will be given (Proposition 3.7).

Case $B$ is perhaps the most interesting part of the whole classification, corresponding to the case where the $\mathbb{Q}$-algebra $A$ mentioned above is a definite quaternion algebra over $\mathbb{Q}$. In this case, if we fix the abstract isomorphism type of the group, the moduli space is isomorphic to the complex projective line $\mathbb{P}^{1}$ (Theorem 3.11). (In the case $G \cong \mathfrak{Q}$, one more trivial component appears corresponding to the natural inclusion $\mathfrak{Q} \subseteq \mathfrak{I}_{\text {。 }}$ )

As a typical example we shall describe the construction when $G$ is isomorphic to the binary tetrahedral group $\mathfrak{T}$. We set

$$
F=\mathbb{Q}[1, i, j, k] \text { and } a=\mathbb{Z}[1, i, j, t], \quad t=(1+i+j+k) / 2 .
$$

Then $F$ is a definite quaternion algebra over $Q$ and $\mathfrak{a}$ is a maximal order of $F$ consisting of Hurwitz quaternions, whose unit group $\mathfrak{a}^{\times}$is isomorphic to $\mathfrak{T}$. Next we set

$$
X=\left\{q \in \mathbb{H} ; q^{2}=-1\right\} \cong \mathbb{P}^{1} \text { (cf。(12))。 }
$$

Then by right multiplications, $X$ parametrizes the complex structures $J(q)$ of $F_{R} \cong \mathbb{H} \cong \mathbb{R}^{4}$, and hence on the real torus $F_{R} / a_{\text {。 }}$ Then the left multiplications on $F_{R}$ induces the action of $\mathfrak{a}^{\times}$on $F_{R} / \mathfrak{a}$ which commutes with each $J(q)$. In this way we get a holomorphic family of special pairs $\left(T_{q}, G_{q}\right)$ with $G_{q} \cong \mathfrak{I}$ parametrized by $X \cong \mathbb{P}^{1}$. Further, if $\tilde{a}^{\times}$is the normalizer of $\mathfrak{a}^{\times}$in the unit group $F^{\times}$, then the inner automorphisms induce the action of $\overline{\mathfrak{a}}^{\times}:=\tilde{\mathfrak{a}}^{\times} / \mathbb{Q}^{\times} \cong \mathfrak{S}_{4}$, the symmetric 
group of degree 4 , on $X$ and it turns out that $Y:=X / \bar{\alpha}^{\times} \cong \mathbb{P}^{1}$ is precisely the moduli space in this case. The construction is quite the same if we modify the definitions of $F$ and $a$ also for $G \cong \mathfrak{Q}$, or $\mathfrak{D}$.

In the above construction the pairs which correspond to the three branch points $p_{i}, 1 \leqq i \leqq 3$, on $Y$ of the covering map $b: X \rightarrow Y$ are important. Indeed, general consideration shows (cf. 2.1, b)) that for the pairs $(T, G)$ not corresponding to these three points $G$ is its own normalizer in Aut $T$, while if $(T, G)$ corresponds to one of the $p_{i,}$ the normalizer $N$ is strictly larger than $G$, and the resulting pair $(T, N)$ is an exceptional pair mentioned earlier; further the structure of the latter can be described explicitly using the above construction (Proposition 3.13). This reduces the study of exceptional pairs to the cases where $G_{0}$ is cyclic, which will be treated in Section 4.

We also show that for a special pair $(T, G)$ in Case $\mathbb{B}$, if $G$ preserves some principal polarization on $T$, then $(T, G)$ is isomorphic to a special pair corresponding to one of the $p_{i}$. This enables us to identify part of our classification with that of [5] already mentioned (cf. Remark 3.18). In fact, we also give in general a classification of special pairs in the generalized sense as in [5]; namely we allow $G$ to be a subgroup of the affine automorphism group of $T$ containing no translations. This needs some extra efforts and occupies the second half of Section 3.

In Section 4 non-special pairs are classified, and the results are summarized in Tables 6 through 11. The case $-1 \in G$ is essential, which we assume now. For the non-exceptional pairs the result is derived in a more or less straightforward way from the results in Section 2 (Tables 6-8). The groups which occur are either abelian with at most two generators, or dihedral of order 8 or 12; in the cyclic case the possible orders of the groups are $2 k$ with $2 \leqq k \leqq 6$. On the other hand, the exceptional pairs $(T, G)$ for which $G$ is maximal in Aut $T$ are exihibited in Table 9; it turns out that there exist eleven such pairs. Non-maximal exceptional pairs and the pairs with $-1 \notin G$ are classified in Tables 10 and 11 respectively.

In the first part of Section 5 we give a result of a classification of rational endomorphism rings $\mathbb{E n d}_{0} T$ of complex tori $T$ of dimension 2. This also tells us the rough structure of Aut $T$ (possibly infinite) for each $T$. 
In the rest of the paper we study special pairs more in detail. Let $a(T), 0 \leqq a(T) \leqq 2$, be the algebraic dimension of $T$, that is, the transcendence degree of its meromorphic function field; thus $a(T)=2$ if and only if $T$ is an abelian surface. We first observe that if $a(T) \leqq 1$, any pair $(T, G)$ is necessarily special (Lemma 3.1). Further, when $a(T)=1$, the result of the classification of the pairs is simple (Proposition 3.10). In particular Aut $T$ is cyclic of order 2, 4, or 6. This in turn implies that in the family of special pairs $\left(T_{q}, G_{q}\right)$ as above

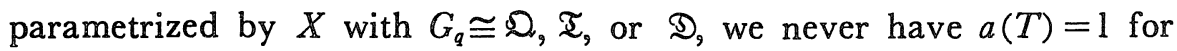
any $q \in X$. More precisely, (e.g., in case of $\mathfrak{T}$ as above), we see that $T_{q}$ is an abelian surface if and only if there exists a non-zero real number $\mu$ such that $\mu q \in F$, and that in this case $T_{q}$ is a singular abelian surface with center $K_{q}$ of $\operatorname{End}_{0} T_{q}$ given by $K_{q}=Q(\mu \sqrt{-1})$, and with $G$-invariant Picard number $\rho^{G}\left(T_{q}\right)=1$; otherwise $a\left(T_{q}\right)=0$ and Picard number $\rho(T)=3$ (Propositions 5.7 and 5.9).

The problem then arises as to which singular abelian surfaces actually appear as $T_{q}$ as above. As for this, we show for example that if $K_{q} \cong Q(\sqrt{-m})$ for a square-free positive integer $m$ we must have $m \not \equiv-1(8)$, and conversely, for any integer $m \geqq 0$ satisfying this condition we can find some $q$ as above such that $K_{q} \cong Q(\sqrt{-m})$ (Proposition 5.13).

In order to get a more definite result, however, first in Section 6 we shall study an interesting relation between special pairs $(T, G)$ and root lattices of rank 2 or 3 . This relation comes from the consideration of the action of $G$ on the second cohomology group $H^{2}(T, \mathbb{Z})$. Namely, let $H^{2}(T, \mathscr{Z})^{G}$ be the sublattice of $G$-invariant elements, and $H^{2}(T, \mathbb{Z})_{G}$ its orthogonal complement in $H^{2}(T, \mathbb{Z})$ considered as a euclidian lattice. Because of the connectedness of the moduli space $Y$ these depend only on the abstract isomorphism class \&s of $G$. In fact, we show that $H^{2}(T, \mathbb{Z})_{G}$ is isomorphic as a euclidian lattice to a root lattice $L_{\mathscr{S}}$ of rank 2 or 3 (Theorem 6.4); more precisely, according as $\mathbb{S}=\mathfrak{C}_{4}, \mathfrak{E}_{6}, \mathfrak{O}, \mathfrak{I}$, or $\mathfrak{S}, L_{\mathbb{S}}$ is the root lattices $A_{1,}^{2}, A_{2}$ $A_{1}^{3}, A_{3}, A_{2} \oplus A_{1}$, which in fact exhaust all the root lattices of rank 2 or 3 up to isomorphisms. (Such isomorphisms will be given explicitly (cf. 6.6)). This implies that we have the natural primitive embedding

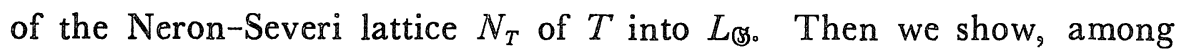
others, that the converse of this is also true; namely, if $N_{T}$ is embed- 
dable as a primitive sublattice in $L_{\mathscr{S}}$ then there exists a subgroup $G$ of Aut $T$ such that $(T, G)$ is a special pair with $G \cong \mathbb{S}$ (Theorem 6.9). In Case $B$ this condition is equivalent to: $\mathbb{N}_{T} \cong L_{\mathscr{G}}$ if $a(T)=0$, and to the existence of a primitive embedding of transcendental lattice $B_{T}$ of $T$ into $L_{\mathscr{S}}(-1)$ if $T$ is a singular abelian surface (cf. Proposition 6.12). For the proof we use the global Torelli theorem for 2marked complex tori due to Shioda [22] and the results of Nikulin [14] [15] concerning the existence and uniqueness of embedding of lattices.

Recall now that by Shioda-Mitani [23] the isomorphism classes of singular abelian surfaces are, via their transcendental lattices, in one to one correspondence with the equivalence classes of oriented binary positive even lattices. By using this and the results obtained in Section 6, we finally obtain in Section 7 a necessary and sufficient condition for a singular abelian surface to admit a special action of $\mathbb{B}(=\mathfrak{D}, \mathfrak{I}$, or $\mathfrak{D})$ in terms of its transcendental lattice (Theorems 7.2 and 7.5). The condition actually depends only on the discriminant form of the lattice, and what we actually get is the classification of those finite quadratic form groups (in terms of the known classification of such objects (cfo [15])) which can be a discriminant form of a transcendental lattice of some singular abelian surface $T$ such that $(T, G)$ is a special pair for some subgroup $G$ of Aut $T$ with $G \cong(\mathbb{S}$. Further, in this case we also obtain the number of conjugacy classes of such $G$ and the degree $\delta=\langle e, e\rangle / 2$ of $(T, G)$, where $\pm e$ are the generators of the $G$-invariant Neron-Severi lattice $N_{T}^{G} \cong \mathbb{Z}$; for instance $\delta=1$ if and only if $G$ preserves some principal polarization of $T$ (Theorems 7.4,7.5). As an illustration we give a table of all the singular abelian surfaces with discriminant $D \leqq 12$ which admit a special \&s-action (cf. Example 7.9).

\section{Notations and Conventions.}

$$
\begin{aligned}
& \mathbb{Z} \quad \text { ring of rational integers } \\
& \mathbb{Q}, \mathbb{R}, \mathbb{C} \text { fields of rational, real, and complex numbers } \\
& \mathbb{H} \text { real quaternion division algebra with standard } \mathbb{R} \text {-basis } \\
& 1, i, j, k \\
& \mathbb{C}^{*}=\mathbb{C}-\{0\}, \quad \mathbb{H}^{\times}=\mathbb{H}-\{0\} \\
& e_{m}=\exp (2 \pi \sqrt{-1} / m), \quad i=e_{4}=\sqrt{-1}, \quad \rho=e_{6}=(1+\sqrt{-3}) / 2
\end{aligned}
$$




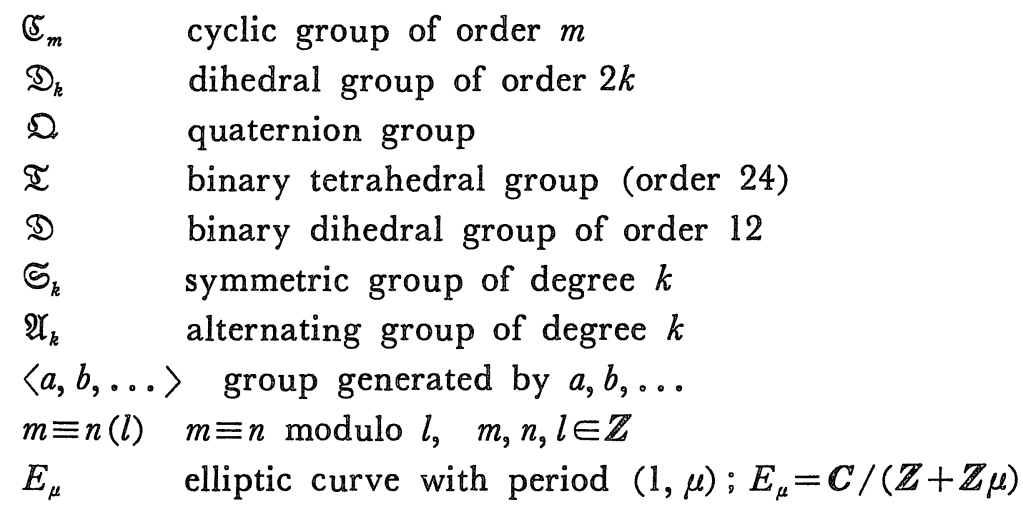

For a ring $R$

$R^{\times} \quad$ group of units of $R$

$M_{k}(R) \quad$ full matrix ring of degree $k$ with coefficients in $R$

$G L_{k}(R)$ group of invertible $k \times k$ matrices with coefficinets in $R$

$(a, b) \quad$ diagonal matrix $\left(\begin{array}{ll}a & 0 \\ 0 & b\end{array}\right)$ in $M_{2}(R)$

$[a, b]$ anti-diagonal matrix $\left(\begin{array}{ll}0 & a \\ b & 0\end{array}\right)$ in $M_{2}(R)$

For groups $H$ and $K$

$M^{K} \quad$ submodule of $K$-invariant elements of a $K$-module $M$

$K \ltimes H, H \rtimes K$ semi-direct product of $H$ by $K$

For any $\mathbb{Z}$-module $M$ and any homomorphism $\lambda: M \rightarrow N$ of $\mathbb{Z}$-modules

$M_{R}=M \otimes_{Z} \mathbb{R}, \quad \lambda_{R}=\lambda \otimes_{Z} \mathbb{R}: M_{R} \rightarrow N_{R}$.

$M^{n}=M^{\oplus n}, \quad K^{n}=K \times \ldots \times K$ ( $n$ times $)$

$T$ complex torus of dimension 2, considered as a complex Lie group with origin $o \in T$

Aut $T$ automorphims group of $T$ (as a complex Lie group)

$-1 \quad$ element of Aut $T$ defined by $(-1)(z)=-z, z \in T$

End $T$ endomorphism ring of $T$

$\operatorname{End}_{0} T=$ End $T \otimes_{Z} Q$ rational endomorphism ring of $T$

$(T, G)$ pair consisting of a complex torus $T$ as above and a finite subgroup $G$ of Aut $T$ with $G \neq\{1\}$.

We call any such pair simply a pair. Two pairs $(T, G)$ and $\left(T^{\prime}, G^{\prime}\right)$ are said to be isomorphic if there exists an isomorphism $u: T \rightarrow T^{\prime}$ of complex Lie groups such that $G^{\prime}=u G u^{-1}$ in Aut $T^{\prime}$.

If $E$ is the tangent space of $T$ at $o$ and $A=H_{1}(T, \mathbb{Z})$, we have the natural identification 


$$
T=E / \Lambda_{0}
$$

This induces two faithful representations $f: G \rightarrow \mathrm{GL}(E)$ and $\nu: G \rightarrow \mathrm{GL}(\Lambda)$, called respectively the complex and rational representations associated to the pair $(T, G)$.

$N_{T}$ Neron-Severi lattice of $T:=\operatorname{Image}$ of $c_{1}: H^{2}\left(T, \mathcal{O}_{1}^{*}\right) \rightarrow H^{2}(T, \mathbb{Z}) B_{T}$ transcendental lattice of $T:=N_{T}^{\frac{1}{T}}$ in $H^{2}(T, \mathbb{Z}) \quad(\mathrm{cf}$ 。 6 )

$\rho(T)$ Picard number of $T:=\operatorname{rank}_{Z} N_{T}$.

Here a lattice, or more precisely, a euclidian lattice is a free $\mathbb{Z}$ module $L$ of finite rank endowed with a $\mathbb{Z}$-valued nondegenerate symmetric bilinear form $\langle\rangle:, L \times L \rightarrow \mathbb{Z} .\langle$,$\rangle is called the inner$ product of $L$.

Other general notations can also be found before Proposition 2.17, after Table 4 , and in 4.1 .

\section{\$2. Moduli Space of Fixed Rational Representation Type}

2.1. a) As is well-known, the moduli space for complex tori does not exist as an analytic space. To remedy this situation one usually consider the marking.

Let $T$ be a complex torus of dimension two. Let $T=E / \Lambda$ be as in (*), where $\Lambda=H_{1}(T, \mathbb{Z})$. Then we get a canonical real linear isomorphism $\lambda: \Lambda_{R} \rightarrow E$. Let $\Lambda^{\circ}=\mathbb{Z}^{4}$. Then a marking of $T$ is an isomorphism $\psi: \Lambda \rightarrow \Lambda^{\circ}$ of $\mathbb{Z}$-modules, and a marked torus is a pair $(T, \psi)$ consisting of a complex torus of dimension 2 and a marking $\psi$ of $T$. We define the isomorphisms of two marked tori in the obvious way. Let $W$ be the set of isomorphism classes of marked tori $(T, \phi)$. Let $i_{*}$ be the multiplication by $\sqrt{-1}$ on $E$. Then any marked complex torus $(T, \phi)$ defines a complex structure $J$ on $\Lambda_{R}^{\circ}$ by $J=\left(\psi_{R} \lambda^{-1}\right) i_{*}\left(\psi_{R} \lambda^{-1}\right)^{-1}$ 。 (Recall that a complex structure on $\Lambda_{R}^{\circ}$ is an endomorphism $J$ of $\Lambda_{R}^{0}$ with $J^{2}=-\mathrm{id}_{\Lambda_{R}^{0} \text { ) }}$ ) Moreover the correspondence $(T, \phi) \rightarrow J$ sets up a bijective correspondence between the set $W$ and the set of complex structures on $\Lambda_{R}^{\circ}$. So in what follows we shall make the identification:

$$
\left.W=\text { complex structure on } \mathscr{A}_{R}^{\circ}\right\} \text { 。 }
$$

In particular $W$ has the natural structure of a homogeneous complex manifold (cf。e.g. [1]): 


$$
W=\mathrm{GL}_{4}(\boldsymbol{R}) / \mathrm{GL}_{2}(\boldsymbol{C}) .
$$

(A homogeneous complex manifold $X=H / K$ is a complex manifold $X$ on which a real Lie group $H$ acts biholomorphically and transitively with $K$ the stabilizer at some point.) Then $\mathrm{GL}_{4}(\mathbb{Z})$ acts on $W$ via the natural inclusion $\mathrm{GL}_{4}(\boldsymbol{Z}) \longrightarrow \mathrm{GL}_{4}(\boldsymbol{R})$. Explicitly, the action takes the form $(T, \psi) \rightarrow(T, h \psi)$, or with respect to the identification (1), the form $J \rightarrow h J h^{-1}, h \in \mathrm{GL}_{4}(\boldsymbol{Z})$.

On the other hand, $W$ parametrizes the universal family $U=(\widetilde{\varpi}: \mathscr{T}$ $\left.\rightarrow W, \tilde{\psi}: R_{1} \widetilde{\varpi}_{*} \mathbb{Z} \rightarrow W \times \Lambda^{\circ}\right)$ of marked complex tori, where $R_{1} \widetilde{\varpi}_{*} \mathbb{Z}$ is the local system with fiber $H_{1}\left(\varpi^{-1}(p), \mathbb{Z}\right), p \in W$. The action of $\mathrm{GL}_{4}(\mathbb{Z})$ on $W$ defined above lifts naturally to the action on the universal family, i. e., to the action on $\mathscr{T}$ making $\varpi$ equivariant.

b) Now we consider an analogue of the above construction in the case of pairs. Let $(T, G)$ be a pair. Let $H$ be any finite subgroup of $\mathrm{GL}_{4}(\boldsymbol{Z})$. Then a marking $\psi$ of type $H$ of $(T, G)$ is a marking $\psi: \Lambda \rightarrow \Lambda^{\circ}$ of $T$ as in a) such that $\psi_{*}(G):=\left\{\phi g \psi^{-1} ; g \in G\right\}=H$. In this case the triple $(T, G ; \phi)$ is called a marked pair of type $H$. The isomorphisms of two marked pairs of type $H$ are defined in the obvious way. Let

$$
W_{H}=\{\text { marked torus of type } H\} / \cong \text {. }
$$

Then $W_{H}$ is naturally a subset of $W$ and with respect to the identification (1) it is given by

$$
W_{H}=\{J \in W ; J h=h J, \forall h \in H\} .
$$

Namely $W_{H}$ is the fixed point set of $H$ if we let $H$ act on $W$ via the inclusion $H \leqq \mathrm{GL}_{4}(\mathbb{Z})$. In particular $W_{H}$ is a complex submanifold of $W$.

Let $\widetilde{\varpi}_{H}: \mathscr{T}_{H} \rightarrow W_{H}$ be the restriction of the universal family $\varpi_{:}: \mathscr{T} \rightarrow W$ to $W_{H}$. Then the induced action of $H$ on $\mathscr{T}_{H}$ preserves each fiber $T_{p}:=\widetilde{\Phi}^{-1}(p), p \in W_{H}$, so that we have the natural embedding $\iota_{p}: H \longrightarrow$ Aut $T_{p}$. Let $G_{p}$ be the image of this embedding. Then

$$
U_{H}:=\left\{\left(T_{p}, G_{p} ; \psi_{p}\right)\right\}_{p \in W_{H}}
$$

is considered as the universal family of marked pairs of type $H$ parametrized by $W_{H}$, where $\psi_{p}: H_{1}\left(T_{p}, \mathbb{Z}\right) \rightarrow \Lambda^{\circ}$ is induced by $\tilde{\phi}$.

Let $(T, G)$ be a pair. If there exists a marking $\phi$ of $(T, G)$ of 
type $H$, then the conjugacy class $(H)$ of $H$ in $\mathrm{GL}_{4}(\mathbb{Z})$ is independent of $\psi$ and depends only on $(T, G)$. We call $(H)$ the rational representation type of $(T, G)$. Let

$$
\mathscr{M}_{(H)}=\{(T, G) \text {; of rational representation type }(H)\} / \cong ;
$$

namely, $\mathscr{M}_{(H)}$ is the moduli space of pairs of fixed rational representation type $(H)$. Let $\pi_{H}: W_{H} \rightarrow \mathscr{M}_{(H)}$ be the natural surjection. Let $N_{H}$ be the normalizer of $H$ in $\mathrm{GL}_{4}(\mathbb{Z})$. Then the induced action of $N_{H}$ on $W$ leaves $W_{H}$ invariant and $\pi_{H}$ induces the natural bijection

$$
N_{H} \backslash W_{H} \cong \mathscr{M}_{(H)} \text { 。 }
$$

Via this bijection $\mathscr{M}_{(H)}$ has the natural quotient topology and, further, if $N_{H}$ acts properly discontinuously on $W_{H}, \mathscr{M}_{(H)}$ even has the natural structure of a normal analytic space. In fact, the isomorphism class of $W_{H}$ as a complex manifold depends only on the conjugacy class $(H)$ but not on the particular choice of $H$. The action $N_{H}$ on $W_{H}$ lifts naturally to an effective action on the universal family $\mathscr{T}_{H} \rightarrow W_{H}$ inducing the "universal action" of $H \subseteq N$ on $\mathscr{T}_{H}$. Therefore if $p$ is any point of $W_{H}$ and $N_{p}$ is the stabilizer at $p$ in $N_{H}$, we have the natural embedding $\hat{\iota}_{p}: N_{p} \longrightarrow$ Aut $T_{p}$ inducing the embedding $\iota_{p}: H \longrightarrow$ Aut $T_{p}$ defined above. Thus if $N(p)$ is the normalizer of $G_{p}$ in Aut $T_{p,}$ then we have the natural identification

$$
N(p)=\hat{\iota}_{p}\left(N_{p}\right) .
$$

2.2. In view of 2.1, in order to determine the structure of the moduli space of pairs in general, we have: 1) to determine all finite subgroups $H$ of $\mathrm{GL}_{4}(\mathbb{Z})$ up to conjugacy for which $W_{H} \neq \emptyset$, and then 2) for any such subgroup $H$, to calculate the normalizer $N_{H}$ of $H$ in $\mathrm{GL}_{4}(\mathbb{Z})$ and to give a description of $W_{H}$ as a complex manifold.

For the first purpose it is convenient to pass from $H$ to another object, a certain quadruple, which is more manageable than $H$ itself. Namely we observe that any subgroup $H$ of $\mathrm{GL}_{4}(\mathbb{Z})$ as above determines a quadruple $F_{H}:=(A, \mathrm{D}, H, M)$ as follows; $\mathrm{A}$ is the $Q$-subalgebra of $M_{4}(Q)$ generated by the elements of $H, \mathfrak{D}=A \cap M_{4}(\mathbb{Z})$, and $M=\Lambda^{\circ}$. Then we see that 1) $A$ is a finite semisimple $Q$-algebra as the homomorphic image of the group algebra of $H$ over $Q, 2) \quad \mathcal{O}$ is an order of $A, \mathrm{i}_{0}$ e., it is a $\mathbb{Z}$-subalgebra of finite $\mathbb{Z}$-rank in $A$ with $0_{Q}=A$ and $\mathfrak{l} \in \mathfrak{D}, 3) H$ is a subgroup of the unit group $\mathfrak{D}^{\times}$of $\left.\mathfrak{D}, 4\right) \mathfrak{D}=\{a \in A$; 
$a M \cong M\}$, where we consider $M$ as a submodule of the $A$-module $M_{\boldsymbol{Q}}$, and finally, 5) any element of $W_{H}$ commutes with elements of $A$ as an endomorphism of $M_{R}$.

In view of this we shall consider such quadruples in general. Namely we consider the quadruples

$$
F=(A, \mathfrak{D}, H, M),
$$

where $A$ is a finite semisimple $\boldsymbol{Q}$-algbera, $\mathfrak{D}$ is an order of $A, H$ is a finite subgroup of $\mathfrak{D}^{\times}$and $M$ is a faithful $\mathbf{D}$-module which is at the same time a free $\mathbb{Z}$-module of rank 4 , and they are required to fulfill the following conditions:

Q1) $A$ is generated by the elements of $H$ as a $Q$-algebra,

Q2) there exists a complex structure $J$ on $M_{R}$ which centralizes $A \subseteq \operatorname{End}_{R} M_{R}$, and

Q3) $\mathfrak{D}=\{a \in A ; a M \subseteq M\}$.

Further for technical reason we also require the following:

Q4) $-1 \in H$ (cf. Remark 2.2 below).

Two such quadruples $(A, \mathfrak{D}, H, M)$ and $\left(A^{\prime}, \mathfrak{o}^{\prime}, H^{\prime}, M^{\prime}\right)$ are said to be isomorphic if there exists an isomorphism $u: M \rightarrow M^{\prime}$ of $\mathbb{Z}$-modules such that the induced homomorphism $u_{*}: \operatorname{End}_{Q} M_{Q} \rightarrow \operatorname{End}_{Q} M_{Q}^{\prime}$ sends the triple $(A, \mathfrak{o}, H)$ onto the triple $\left(A^{\prime}, \mathfrak{o}^{\prime}, H^{\prime}\right)$ with respect to the natural inclusions $H \subseteq \mathfrak{D} \subseteq A \subseteq \operatorname{End}_{Q} M_{Q}$ and $H^{\prime} \subseteq \mathfrak{o}^{\prime} \subseteq A^{\prime} \subseteq \operatorname{End}_{Q} M_{Q}^{\prime}$.

Now we denote by $\mathscr{Q}$ the set of quadruples as above and by $\overline{2}$ the set of isomorphism classes of quadruples in 2 . Then the quadruple $F_{H}$ determined by $H \subseteq \mathrm{GL}_{4}(\boldsymbol{Z})$ as above is naturally considered as an element of 2 . For any quadruple $F$ in $\mathscr{2}$ we define the normalizer $N_{F}$ of $F$ by

$$
N_{F}=\left\{g \in \mathrm{Aut}_{Z} M ; g \mathrm{Ag}^{-1}=A, g \emptyset g^{-1}=\mathfrak{D}, g H g^{-1}=H\right\} .
$$

We also define the set $W_{F}$ by the set of all $A_{R}$-linear complex structures on $M_{R}$;

$$
W_{F}=\left\{J \in \operatorname{End}_{A_{R}} M_{R} ; J^{2}=-\mathrm{id}_{M_{R}}\right\} .
$$

Up to isomorphisms $N_{F}$ and $W_{F}$ depend only on the isomorphism class of $F$. From the definitions the following is immediate to see.

Lemma 2.1. The above correspondence $H \rightarrow F_{H}$ sets up a natural bijective correspondence between a) the set $\mathscr{H}$ of finite subgroups $H$ of 
$\mathrm{GL}_{4}(\mathbb{Z})$ with $-1 \in H$ and with $W_{H} \neq \varnothing$, and $\left.\mathrm{b}\right)$ the set 2 of quadruples as defined above; it also induces one between a)' the set of conjugacy classes of the groups in $\mathscr{H}$ and $\mathrm{b})^{\prime}$ the set $\overline{\mathscr{Q}}$ of isomorphism classes of quadruples in 2. Under the first correspondence the normalizer $N_{H}$ of $H$ in $\mathrm{GL}_{4}(\mathbb{Z})$ and the normalizer $N_{F}$ of $F$ defined above (resp. the set of complex structures $W_{H}$ and $W_{F}$ ) are naturally identified (up to isomorphisms). In particular we have the natural identification $\mathscr{M}_{(H)}=\mathscr{M}_{F}:=N_{F} \backslash W_{F}$, where $F=F_{H^{\circ}}$

Remark 2.2. The condition $-1 \in H$ corresponds to considering only those pairs $(T, G)$ with $-1 \in G$. In fact, any pair $\left(T, G^{\prime}\right)$ with $-1 \notin G^{\prime}$, gives rise cannonically to the pair $(T, G)$ with $-1 \in G$ by setting $G=\left\langle G^{\prime},-1\right\rangle$, and in this way the classification of such pairs are readily deduced from that of pairs with $-1 \in G$ (cf. 4.4).

Definition. Let $(T, G)$ be a pair with $-1 \in G$. Let $(H)$ be the rational representation type of $(T, G)$. Then any quadruple in 2 whose isomorphism class in $\overline{\mathscr{Q}}$ corresponds to the class $(H)$ in the correspondence of Lemma 2.1 will be called a quadruple associated to $(T, G)$.

Let $F=(A, \mathfrak{D}, H, M)$ be a quadruple in 2 . Via the identification of $W_{F}$ with $W_{H}$ in Lemma 2.1 we can put on $W_{F}$ the natural structure of a complex manifold. Moreover the universal family (2) gives rise to a holomorphic family

$$
U_{F}=\left\{\left(T_{p}, G_{p}\right)\right\}_{p \in W_{F}}
$$

of pairs parametrized by $W_{F}$, where we have neglected the marking $\psi_{p}$. We call $U_{F}$ the holomorphic family associated to $F$ (parametrized by $W_{F}$ ). In view of the universality of (2) and Lemma 2.1 the following lemma is obvious.

Lemma 2.3. Let $F$ be as above. Then for any pair $(T, G)$ with the associated quadruple isomorphic to $F$, there exists a point $p$ of $W_{F}$ such that $(T, G)$ is isomorphic to $\left(T_{p}, G_{p}\right)$. Moreover for $p, p^{\prime} \in W_{F},\left(T_{p}, G_{p}\right)$ and $\left(T_{p^{\prime}}, G_{p^{\prime}}\right)$ are isomorphic if and only if there exists an element $\gamma$ of $N_{F}$ such that $p=\gamma\left(p^{\prime}\right)$. 
2. 3. In view of 2.2 our first task should be the classification of the quadruples $(A, 0, H, M)$ in 2 , which will eventually be summarized in Proposition 2. 14 below.

a) We start with the determination of the structure of $A$.

Lemma 2.4. Let $(A, \mathfrak{D}, H, M)$ be a quadruple in 2. Then one of the following is true: 1) $A$ is commutative; more precisely, $A$ is a field of degree $\leqq 4$ or a direct sum of two fields of degree $\leqq 2,2) A$ is a quaternion algebra over $\boldsymbol{Q}$, and 3 ) $A \cong M_{2}(K)$ for some imaginary quadratic field $K$.

Proof. Let $A=A_{1} \oplus \ldots \oplus A_{k}$ be a direct sum decomposition of $A$ into simple factors $A_{i}$. Write $1=e_{1}+\ldots+e_{k}$ with $e_{i} \in A_{i}$. Let $V=M_{Q}$ and $V_{i}=e_{i} V$. Then we have the direct sum decomposition $V=V_{1} \oplus \ldots$ $\oplus V_{k}$ and the natural embeddings $A_{\imath} \longrightarrow$ End $V_{i}$. Let $J$ be as in $\left.Q 2\right)$. Then, since $J$ commutes with $e_{i,} V_{i, \boldsymbol{R}}$ are $J$-invariant and hence of even dimension over $\boldsymbol{R}$. Thus $k \leqq 2$ and if the equality holds, we have $\operatorname{dim}_{Q} V_{i}=2$ so that $A_{i}$ is a field of degree $\leqq 2$. Suppose then that $k=1$, i. e., $A$ is simple. If $A$ is a division algebra, then $V$ is a vector space over $A$ so that $\operatorname{rank}_{Q} A \leqq 4$. Thus $A$ belongs to the cases 1) or 2). Suppose that $A$ is not a division algebra so that $A \cong M_{2}(K)$ for some division algebra $K$ over $Q$. Write $1=e_{1}+e_{2}$ with $e_{1}=\left(\begin{array}{ll}1 & 0 \\ 0 & 0\end{array}\right)$ and $e_{2}=\left(\begin{array}{ll}0 & 0 \\ 0 & 1\end{array}\right)$. Then again we have $V=V_{1} \oplus V_{2}$ with $V_{i}=e_{i} V$ and with $V_{i, \boldsymbol{R}} J$-invariant. In particular $V_{i, \boldsymbol{R}}$ has a natural complex structure. Then $K$ can be embedded both in End $V_{i}$ and in $\operatorname{End}_{C} V_{i, R} \cong C$. It follows that $K$ is an imaginary quadratic field if $K \nsubseteq Q$. The lemma is proved.

b) Next, in Lemmas 2.5,2.7 and 2.10 below we shall give the possible types of $(A, \mathfrak{D}, H)$ for quadruples $(A, \mathfrak{D}, H, M)$ in $\mathscr{Q}$, in case $\operatorname{rank}_{Q} A \leqq 4$, i. e., $A$ is either commutative or is a quaternion algebra over $Q$. We define two such triples $(A, \mathfrak{D}, H)$ and $\left(A^{\prime}, \mathfrak{D}^{\prime}\right.$, $H^{\prime}$ ) to be isomorphic if there exists an algebra isomorphism $u: A \rightarrow A^{\prime}$ such that $u(\mathfrak{D})=\mathfrak{o}^{\prime}$ and $u(H)=H^{\prime}$.

bl) First we consider the case where $A$ is commutative. For the statement of the result we need some definitions and notation: $\tilde{\mathfrak{D}}$ 
denotes the maximal order of $A$. Then $\tilde{\mathfrak{D}}_{f}^{\times}$denotes the subgroups of elements of finite order in the unit group $\tilde{\mathfrak{d}}^{\times}$. Let $m=1,2$ or 3. Then we set

$$
R_{m}=\mathbb{Z}\left[e_{2 m}\right] . \quad\left(R_{1}=\mathbb{Z}_{\circ}\right)
$$

Then if $A \cong \mathbb{Q}\left(e_{2 m}\right) \oplus \mathbb{Q}\left(e_{2 n}\right)$, we have $\tilde{\mathfrak{D}}=R_{m} \oplus R_{n}$. Let $\mathbb{F}_{m}=\mathbb{Z} / m \mathbb{Z}$, considered as a finite field. Denote by $\Delta$ the diagonal in general. When $A=\mathbb{Q} \oplus \mathbb{Q}\left(e_{2 m}\right), m=2,3$ we define

$$
\hat{\mathrm{o}}_{m}=\hat{p}^{-1}(\Delta)
$$

where $\hat{p}: \tilde{D} \cong \mathbb{Z} \oplus R_{m} \rightarrow \mathbb{Z} / m \mathbb{Z} \oplus R_{m} /\left(1+e_{2 m}\right) R_{m} \cong \mathbb{F}_{m}^{2}$ is the natural projection. When $A=\mathbb{Q}\left(e_{2 m}\right)^{2}$; we define

$$
\mathfrak{o}_{m}=p^{-1}(\Delta) \text { and } \mathfrak{o}_{m}^{\prime}=p^{\prime-1}(\Delta)
$$

where

and

$$
p: \tilde{\mathrm{D}} \cong R_{m}^{2} \rightarrow \tilde{\mathrm{D}} /\left(1+e_{2 m}\right) \tilde{\mathrm{D}} \cong \mathbb{F}_{m}^{2}, \quad m=2,3,
$$

$$
p^{\prime}: \tilde{\mathfrak{D}} \cong R_{m}^{2} \rightarrow \tilde{\mathrm{D}} / 2 \tilde{\mathrm{D}} \cong\left(R_{m} / 2 R_{m}\right)^{2}, \quad 1 \leqq m \leqq 3,
$$

are the natural projections.

Lemma 2.5. Let $(A, \mathfrak{0}, H, M)$ be a quadruple in 2 with $A$ commutative. Then $(A, \mathbb{0}, H)$ is isomorphic to one of the following triples: $\left(\mathbb{Q}\left(e_{2 m}\right), \tilde{\mathfrak{D}}, \tilde{\mathfrak{D}}_{f}^{\times}\right), 1 \leqq m \leqq 6, \quad\left(\mathbb{Q}^{2}, \mathfrak{D}, \tilde{\mathfrak{D}}^{\times}\right), \quad \mathfrak{D}=\tilde{\mathfrak{D}}, \mathfrak{o}_{1}^{\prime}, \quad\left(\mathbb{Q} \oplus \mathbb{Q}(i), \mathfrak{D}, \tilde{\mathfrak{D}}^{\times}\right), \quad \mathfrak{D}=$ $\tilde{\mathfrak{D}}, \hat{\mathfrak{D}}_{2},\left(\mathbb{Q} \oplus \mathbb{Q}(\rho), \tilde{\mathfrak{D}}, \tilde{\mathfrak{D}}^{\times}\right), \quad\left(\mathbb{Q} \oplus \mathbb{Q}(\rho), \quad \mathfrak{D}, \hat{\mathfrak{D}}_{3}^{\times}\right), \quad \mathfrak{D}=\tilde{\mathfrak{D}}, \hat{\mathfrak{D}}_{3},\left(\mathbb{Q}(i)^{2}, \mathfrak{D}, \tilde{\mathfrak{D}}^{\times}\right)$, $\mathfrak{D}=\tilde{\mathfrak{D}}, \mathfrak{o}_{2},\left(\mathbb{Q}(i)^{2}, \tilde{\mathfrak{D}}, \mathfrak{D}_{2}^{\prime \times}\right), \quad \mathfrak{D}=\tilde{\mathfrak{D}}, \mathfrak{o}_{2}, \mathfrak{D}_{2}^{\prime}, \quad\left(\mathbb{Q}(\rho)^{2}, \tilde{\mathfrak{D}}, \tilde{\mathfrak{D}}^{\times}\right),\left(\mathbb{Q}(\rho)^{2}, \mathfrak{D}, \mathfrak{o}_{3}^{\times}\right)$, $\mathfrak{D}=\tilde{\mathfrak{D}}, \mathfrak{D}_{3},\left(\mathbb{Q}(\rho)^{2}, \mathfrak{D}, \mathfrak{D}_{3}^{\prime \times}\right), \mathfrak{D}=\tilde{\mathfrak{D}}, \mathfrak{o}_{3}^{\prime},\left(\mathbb{Q}(i) \oplus \mathbb{Q}(\rho), \tilde{\mathfrak{D}}, \tilde{\mathfrak{D}}^{\times}\right)$.

Proof. By Lemma 2.4 $A=K$ or $K_{1} \oplus K_{2}$, where $K$ and $K_{i}$ are fields of degree $d \leqq 4$ and $d_{i} \leqq 2$ respectively. Since $A$ is generated by elements of finite order, $K$ and $K_{i}$ must be cyclotomic fields. From these, the assertion on $A$ follows.

Now suppose first that $A=\mathbb{Q}\left(e_{2 m}\right)$. If $\mathfrak{o}$ is not the maximal order, then $H$ contains neither a primitive $2 m$-th root of unity nor its minus, so that $\mathbb{Z}$-rank of $\mathfrak{D} \leqq 2$, a contradiction. Thus $\mathfrak{D}$ is the maximal order of $A$. Similarly, $H$ must contain a primitive $2 m$-th root of unity; hence $H=\tilde{D}_{f}^{x}$.

Next suppose that $A=\mathbb{Q}\left(e_{2 m}\right) \oplus \mathbb{Q}\left(e_{2 n}\right)$ with $1 \leqq m \leqq n \leqq 3$. We first enumerate the subgroups $H$ of $\tilde{\mathfrak{D}}^{\times} \cong \mathfrak{E}_{2 m} \times \mathfrak{夭}_{2 n}$ which generate $A$ as $\mathbb{Q}$ - 
algebras; they are one of the following: 1) $\left.\tilde{\mathfrak{D}}^{\times}, 2\right)\langle(-1, \rho)\rangle \subseteq$ $\boldsymbol{Q} \oplus \boldsymbol{Q}(\rho), \quad 3) H_{3}:=\left\langle-1,\left(\rho^{2}, 1\right),\left(1, \rho^{2}\right)\right\rangle \subseteq \boldsymbol{Q}(\rho)^{2}, \quad$ 4) $H_{m}^{\prime}:=\langle(1, \pm 1)$, $\left.\left.\left(e_{2 m}, e_{2 m}\right)\right\rangle \subseteq Q\left(e_{2 m}\right)^{2}, 5\right)\left\langle(1, \pm 1),\left(\rho^{2}, \rho^{-2}\right)\right\rangle \subseteq Q(\rho)^{2}$. Here $H_{3}^{\prime}$ and the group in 5) are transformed to each other by the complex conjugation on the second factor. So we may omit the case 5) from our consideration. We next determine for each group $H$ as above the order $\mathfrak{D}$ of $A$ generated by $H$. The resulting triples $(A, \mathfrak{D}, H)$ are as follows: $\left(\boldsymbol{Q}^{2}, \mathfrak{o}_{1}^{\prime}, \tilde{\mathfrak{o}}^{\times}\right),\left(\boldsymbol{Q} \oplus \boldsymbol{Q}(i), \hat{\mathrm{D}}_{2}, \tilde{\mathrm{o}}^{\times}\right),\left(\boldsymbol{Q} \oplus \boldsymbol{Q}(\rho), \tilde{\mathrm{D}}, \tilde{\mathrm{o}}^{\times}\right),(\boldsymbol{Q} \oplus \boldsymbol{Q}(\rho)$, $\left.\hat{\mathfrak{D}}_{3},\langle(-1, \rho)\rangle\right),\left(\boldsymbol{Q}(i)^{2}, \mathfrak{o}_{2}, \tilde{\mathfrak{D}}^{\times}\right),\left(\boldsymbol{Q}\left(e_{2 m}\right)^{2}, \mathfrak{o}_{m}^{\prime}, H_{m}^{\prime}\right), m=2,3,\left(\boldsymbol{Q}(\rho)^{2}, \tilde{\mathfrak{D}}, \tilde{\mathfrak{D}}^{\times}\right)$, $\left(\boldsymbol{Q}(\rho)^{2}, \mathfrak{D}_{3}, H_{3}\right), \quad\left(\boldsymbol{Q}(i) \oplus \boldsymbol{Q}(\rho), \tilde{\mathfrak{D}}, \tilde{D}^{\times}\right)$. Moreover in all the cases we have $H=\mathfrak{D}^{\times}$, the unit group of $\mathfrak{D}$. From this, together with the fact that $\tilde{\mathfrak{D}}, \mathfrak{o}_{m}, \mathfrak{o}_{m}^{\prime}$ (resp. $\tilde{\mathfrak{D}}, \hat{\mathfrak{D}}_{m}$ ) are unique orders in $A$ containing $\mathfrak{o}_{m}^{\prime}$ (resp. $\tilde{\mathrm{D}}_{m}$ ), the lemma follows immediately in this case also.

q. e. d.

b2) Next we consider the case of a definite quaternion algebra. Let $\boldsymbol{H}$ be the real quaternion division algebra over $\boldsymbol{R}$ with the istandard basis $1, i, j, k$. We set

$$
\begin{aligned}
& F=Q[1, i, j, k], \quad \mathfrak{a}=\mathbb{Z}[1, i, j, t], \quad \mathfrak{a}_{0}=\mathbb{Z}[1, i, j, k] \\
& F^{\prime}=\boldsymbol{Q}[1, i, \sqrt{3} j, \sqrt{3} k], \quad \mathfrak{b}=\mathbb{Z}[1, i, h, l]
\end{aligned}
$$

where

$$
t=(1+i+j+k) / 2, \quad h=(i+\sqrt{3} j) / 2, \quad l=(1+\sqrt{3} k) / 2 .
$$

Then $F$ and $F^{\prime}$ are definite quaternion algebras over $Q$, and $\mathfrak{a}_{,} a_{0}$ and $\mathfrak{b}$ are orders of $F$ and $F^{\prime}$ respectively.

Lemma 2.6. 1) $\mathfrak{a}$ and $\mathfrak{b}$ are maximal orders of $F$ and $F^{\prime}$ respectively. 2) The orders of $F$ which contain $a_{0}$ are just $a_{0}$ and $a_{0}$ 3) The groups of units of these orders are given respectively by

$$
\begin{array}{ll}
\mathfrak{a}_{0}^{\times}=\{ \pm 1, \pm i, \pm j, \pm k\} & \cong \mathfrak{Q} \\
\mathfrak{a}^{\times}=\{ \pm 1, \pm i, \pm j,( \pm 1 \pm i \pm j \pm k) / 2\} & \cong \mathfrak{I} \\
\mathfrak{b}^{\times}=\{ \pm 1, \pm i, \pm h, \pm l, \pm i h, \pm i l\} & \cong \mathfrak{D} .
\end{array}
$$

Proof. See e.g., Dickson [4]: p. 172, Satz 1 for 1) and 2), and p. 182 (20) and p. 192 (28) for 3). 
Lemma 2.7. Let $(A, \mathfrak{D}, H, M)$ be a quadruple in 2. Suppose that $A$ is a definite quaternion algebra. Then $(A, \mathfrak{D}, H)$ is isomorphic to one of the following triples; 1) $\left.\left.\left(F, \mathfrak{a}, \mathfrak{a}^{\times}\right), 2\right)\left(F, \mathfrak{a}, \mathfrak{a}_{0}^{\times}\right), 3\right)\left(F, \mathfrak{a}_{0}, \mathfrak{a}_{0}^{\times}\right)$and 4) $\left(F^{\prime}, \mathfrak{b}, \mathfrak{b}^{\times}\right)$.

Proof. $H$ is not commutative since $A$ is not. Let $\tilde{\mathfrak{s}}$ be any maximal order containing $\mathfrak{D}$. Since $\tilde{\mathfrak{D}}$ contains the noncyclic group $H$, it follows that $A \cong F$ or $F^{\prime}$; moreover the maximal orders of $A$ are conjugate to each other (cf. Vignera [26], p. 145, Prop. 3.1 and p. 26, Cor. 4.11). Hence we may assume that the above isomorphism sends $\tilde{D}$ onto $\mathfrak{a}$ or $\mathfrak{b}$ respectively. Then $H$, being noncyclic, must be isomorphic to $\mathfrak{I}$ or $\mathscr{Q}($ resp. $\mathfrak{D})$ if $A \cong F$ (resp. $\left.F^{\prime}\right)$. This, combined with Lemma 2. 6 , gives the lemma immediately.

In passing we note also the following fact (cf. Vignera [26], I. 3. 1) for later use.

Lemma 2.8. Let $\tilde{\mathfrak{a}}^{\times}, \tilde{\mathfrak{a}}_{0}^{\times}\left(\right.$resp. $\left.\tilde{\mathfrak{b}}^{\times}\right)$be the normalizer of $\mathfrak{a}^{\times}, \mathfrak{a}_{0}^{\times}$(resp. $\left.\mathfrak{b}^{\times}\right)$in $F^{\times}\left(r e s p . F^{\prime \times}\right)$. Then we have $\tilde{\mathfrak{a}}^{\times}=\tilde{\mathfrak{a}}_{0}^{\times}=\left\langle{\mathfrak{Q}^{\times}}^{\times}, \mathfrak{a}^{\times}, 1+i\right\rangle$, and $\tilde{b}^{\times}=\left\langle\mathfrak{Q}^{\times}, \mathfrak{b}^{\times}, \sqrt{3}+k\right\rangle$.

Let $F_{1}^{\times}$(resp. $\left.F_{1}^{\prime \times}\right)$ be the multiplicative group of elements of unit norm of $F$ (resp. $F^{\prime}$ ). Then it follows from the above lemma that

$$
\left\{\begin{array}{l}
\tilde{\mathfrak{a}}^{\times} \cap F_{1}^{\times}=\tilde{\mathfrak{a}}_{0}^{\times} \cap F_{1}^{\times}=\left\langle\mathfrak{a}^{\times},(1+i) / \sqrt{2}\right\rangle \cong \mathfrak{O}, \quad \text { and } \\
\tilde{\mathfrak{b}}^{\times} \cap F_{1}^{\times}=\left\langle\mathfrak{b}^{\times},(\sqrt{3}+k) / 2\right\rangle \cong \mathfrak{D}
\end{array}\right.
$$

where $\mathfrak{D}$ is the binary octahedral group and $\mathfrak{D}$ is the binary dihedral group of order 24 .

b3) Finally we consider the case of indefinite quaternion algebras. We take the following presentation of the dihedral group $\mathscr{D}_{k}$ of order $2 k ; \mathfrak{D}_{k}=\left\{\sigma, \tau ; \sigma^{k}=\tau^{2}=1, \tau \sigma \tau=\sigma^{-1}\right\}$. For $k=4,6$ we define the subgroup $\hat{D}_{k}$ of $\mathrm{GL}_{2}(\mathbb{Z})$ isomorphic to $\mathfrak{D}_{k}$ by:

$$
\left\{\begin{array}{l}
\hat{D}_{4}:\left\langle\tau=\left(\begin{array}{ll}
0 & 1 \\
1 & 0
\end{array}\right), \sigma=\left(\begin{array}{rr}
0 & 1 \\
-1 & 0
\end{array}\right)\right\rangle \\
\hat{D}_{6}:\left\langle\tau=\left(\begin{array}{ll}
0 & 1 \\
1 & 0
\end{array}\right), \sigma=\left(\begin{array}{rr}
0 & -1 \\
1 & 1
\end{array}\right)\right\rangle .
\end{array}\right.
$$

We note that such realization of $\mathfrak{D}_{k}$ is unique up to conjugations in 
$\mathrm{GL}_{2}(\boldsymbol{Z})$. Let $\mathfrak{a}_{k}, k=4,6$, be the orders of $M_{2}(\boldsymbol{Q})$ generated by the elements of $\hat{D}_{k}$. Then $\mathfrak{a}_{k}$ are free $\boldsymbol{Z}$-modules of rank 4 with free bases given respectively by

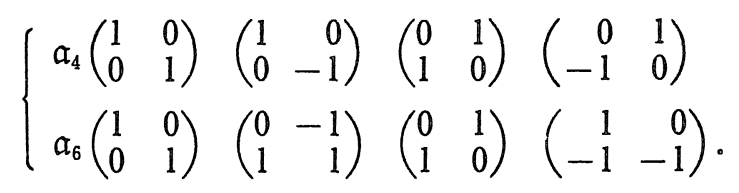

Then

$$
\mathfrak{a}_{4}^{\prime}:=\mathfrak{a}_{4}\left[\left(\begin{array}{ll}
1 & 1 \\
0 & 0
\end{array}\right)\right],
$$

the $a_{4}$-module generated by $\left(\begin{array}{ll}1 & 1 \\ 0 & 0\end{array}\right)$, also is an order of $M_{2}(\boldsymbol{Q})$. From (7) we have

and

$$
\left\{\begin{array}{l}
\mathfrak{a}_{4}=\left\{\left(\begin{array}{ll}
a & b \\
c & d
\end{array}\right) \in M_{2}(\mathbb{Z}) ; a \equiv d, \quad b \equiv c(2)\right\} \\
\mathfrak{a}_{4}^{\prime}=\left\{\left(\begin{array}{ll}
a & b \\
c & d
\end{array}\right) \in M_{2}(\mathbb{Z}) ; a+b+c+d \equiv 0(2)\right\} \\
\mathfrak{a}_{6}=\left\{\left(\begin{array}{ll}
a & b \\
c & d
\end{array}\right) \in M_{2}(\mathbb{Z}) ; a+b \equiv c+d(3)\right\}
\end{array}\right.
$$

$$
\left\{\begin{array}{l}
2 M_{2}(\boldsymbol{Z}) \leqq \mathfrak{a}_{4} \leqq \mathfrak{a}_{4}^{\prime} \subseteq M_{2}(\mathbb{Z}) \\
3 M_{2}(\boldsymbol{Z}) \leqq \mathfrak{a}_{6} \leqq M_{2}(\boldsymbol{Z})
\end{array}\right.
$$

Lemma 2.9. Let $\mathfrak{b}_{k}, k=4$ or 6 , be an order of $M_{2}(\mathbb{Q})$ between $\mathfrak{a}_{k}$ and $M_{2}(\mathbb{Z})$. Then $\mathfrak{b}_{4}=\mathfrak{a}_{4}, \mathfrak{a}_{4}^{\prime}$ or $M_{2}(\mathbb{Z})$ and $\mathfrak{b}_{6}=\mathfrak{a}_{6}$ or $M_{2}(\mathbb{Z})$.

Proof. Let $m=k / 2$. By (9) the problem is reduced to determining the subalgebras of $M_{2}\left(\mathbb{F}_{m}\right) \cong M_{2}(\mathbb{Z}) / m M_{2}(\boldsymbol{Z})$ containing the image $\overline{\mathfrak{a}}_{k}$ of $\mathfrak{a}_{k}$. Since $\operatorname{dim}_{F_{3}} \overline{\mathfrak{a}}_{6}=3$ we have $\mathfrak{b}_{6}=\mathfrak{a}_{6}$ or $M_{2}(\mathbb{Z})$. On the other hand, $\bar{a}_{4}=\mathbb{F}_{2}\left[\left(\begin{array}{ll}0 & 1 \\ 1 & 0\end{array}\right)\right]$ and then direct computation shows the desired assertion.

Using this we shall obtain the following:

Lemma 2.10. Let $(A, \mathfrak{D}, H, M)$ be a quadruple in 2. Suppose that $A$ is an indefinite quaternion algebra over $\boldsymbol{Q}$. Then $(A, \mathfrak{D}, H)$ is isomorphic to one of the following triples; 1) $\left.\left(M_{2}(\mathbb{Q}), M_{2}(\mathbb{Z}), \hat{D}_{4}\right), 2\right)\left(M_{2}(\mathbb{Q})\right.$, $\left.\left.\left.\left.\mathfrak{a}_{4}^{\prime}, \hat{D}_{4}\right), 3\right)\left(M_{2}(\boldsymbol{Q}), \mathfrak{a}_{4}, \hat{D}_{4}\right), 4\right)\left(M_{2}(\boldsymbol{Q}), M_{2}(\boldsymbol{Z}), \hat{D}_{6}\right), 5\right)\left(M_{2}(\boldsymbol{Q}), \mathfrak{a}_{6}, \hat{D}_{6}\right)$. 
Proof. Consider $H$ as a finite subgroup of $\mathrm{GL}_{2}(\mathbb{R}) \cong A_{\mathbb{R}}^{\times}$, and hence $A$ as a finite $\mathbb{Q}$-subalgebra of $M_{2}(\mathbb{R}) \cong A_{R}$. Then we see that $H$ is either cyclic or dihedral. $H$ is not commutative since $A$ is not. Hence $H$ is dihedral. Moreover since the trace of any element of $H$ is in $Q$, it has order $\leqq 6, \neq 5$. Thus $H$ is isomorphic to $\mathfrak{D}_{4}$ or to $\mathfrak{D}_{6}$. After conjugation in $\mathrm{GL}_{2}(\mathbb{R})$ we may assume that $H$ is realized in $M_{2}(\mathbb{Z})$ as in (6) since $\mathfrak{D}_{k}$ embedded in $\mathrm{GL}_{2}(\mathbb{R})$ are conjugate to each other. This then implies that $A$ coincides with $M_{2}(\mathbb{Q})$. Fix a maximal order $\tilde{a}$ of $M_{2}(\mathbb{Q})$ which contains $H$. Since the maximal order of $M_{2}(Q)$ are conjugate to each other (cf. [26], p. 28, 4.2), after conjugation in $M_{2}(\mathbb{Q})$ we may further assume that $\tilde{a}=M_{2}(\mathbb{Z})$. Even after this, by the conjugacy equivalence of realizations of $\mathfrak{D}_{k}$ in $\mathrm{GL}_{2}(\mathbb{Z})$ mentioned above, we may assume that $H$ is realized in $M_{2}(\mathbb{Z})$ as in (6). Then the lemma follows from Lemma 2.9.

Analogously to Lemma 2.8, we get by direct computations the following:

Lemma 2.11. Let $\alpha_{4}=\left(\begin{array}{rr}-1 & 1 \\ 1 & 1\end{array}\right)$ and $\alpha_{6}=\left(\begin{array}{rr}-1 & -2 \\ 2 & 1\end{array}\right)$. Then the normalizer $\check{D}_{k}$ of $\hat{D}_{k}, k=4,6$, in $\mathrm{GL}_{2}(Q)$ are given by $\check{D}_{k}=\left\langle Q^{\times}, \hat{D}_{k}, \alpha_{k}\right\rangle$.

2.4. We shall finally deal with $M$. Let $(A, \mathfrak{D}, H, M)$ be a quadruple in 2. A lattice $\Lambda$ in $A$, i. e., a free $\mathbb{Z}$-submodule of $A$ with $\Lambda_{Q}=A$, is called a proper $\mathfrak{0}$-ideal if $\mathfrak{0}=\{a \in A ; a \Lambda \subseteq \Lambda\}$. It is called principal if $\Lambda=\mathfrak{o} \xi$ for some element $\xi \in A$. First we show the following:

Lemma 2.12. Let $(A, \mathrm{D}, H, M)$ be a quadruple in 2. Assume that $\operatorname{rank}_{Q} A=2$ or 4 . Then any proper $\mathrm{D}$-ideal of $A$ is principal except the cases $\mathfrak{D}=\mathfrak{p}_{1}^{\prime}$.

Proof. Suppose first that $\mathfrak{D}$ is a maximal order of $A$. We then have to distinguish four cases according to the structure of $A: 1$ ) $\left.\left.Q\left(e_{2 m}\right), 1 \leqq m \leqq 6,2\right) \mathbb{Q}\left(e_{2 m}\right) \oplus \mathbb{Q}\left(e_{2 n}\right), 1 \leqq m \leqq n \leqq 3,3\right) F$ or $F^{\prime}$, and 4$)$ $M_{2}(Q)$. In the cases 1),3) and 4) the result is well-known. (See, e. g., Hasse [10], p. 594 for the case 1), [26], p. 145, Prop. 3.1 for 3) and [26], p. 28, 4. 2 for 4).) In the case 2) let $\Lambda$ be any proper $\tilde{\mathfrak{D}}^{-}$ ideal of $A_{0}$ Since $e_{1}:=(1,0)$ and $e_{2}=(0,1)$ are in $\tilde{0}$, it follows 
that $\Lambda$ is the direct sum $\Lambda=\Lambda_{1} \oplus \Lambda_{2}$ with $\Lambda_{i}=e_{i} \Lambda$; further each $\Lambda_{i}$ is a fractional ideal of $K_{i}$, which is principal in $K_{i}$ as we have noted above. Thus $A$ also is principal.

So we may assume that $\mathfrak{D}$ is not a maximal order with $0 \neq 0_{1}^{\prime}$. The cases to be considered are: $\left.(A, \mathfrak{D})=1)\left(\mathbb{Q}\left(e_{2 m}\right)^{2}, \mathfrak{D}_{m}\right), m=2,3,2\right)$ $\left.\left.\left(\boldsymbol{Q}(\rho)^{2}, \mathfrak{b}_{3}^{\prime}\right), 3\right)\left(F, \mathfrak{a}_{0}\right), 4\right)\left(M_{2}(\boldsymbol{Q}), \mathfrak{b}\right)$ with $\mathfrak{D}=\mathfrak{a}_{4}, \mathfrak{a}_{4}^{\prime}$ or $\mathfrak{a}_{6}$. In view of (4) and (9) we have the canonical inclusion $\mathfrak{D} \longrightarrow \tilde{\mathfrak{d}}$ of $\mathfrak{D}$ into a maximal order $\tilde{\mathfrak{D}}$ of $A$ (which is of course unique if $A$ is commutative); further we can find a nonzero element $\alpha$ in the center of $\tilde{\mathfrak{D}}$ such that $\alpha \tilde{\mathfrak{D}} \cong \mathfrak{D} \cong \tilde{D}$; we may indeed take $\alpha:=1) 1+e_{2 m}$, 2) 2,3$) 2$ and 4) 2 or 3 , in respective cases. Let $\hat{\mathfrak{b}}=\tilde{\mathfrak{D}} / \alpha \tilde{\mathfrak{D}}$ and $\overline{\mathfrak{D}}=\mathfrak{o} / \alpha \tilde{\mathfrak{D}}$. These are finite $\boldsymbol{C}$-algebras.

Now let $\Lambda$ be any proper $\mathfrak{D}$-ideal of $A$. Let $\tilde{\Lambda}=\tilde{D} \Lambda$. Then $\tilde{\Lambda}$ is a (proper) $\tilde{\mathfrak{D}}$-ideal and we have $\alpha \tilde{\Lambda} \cong \Lambda \subseteq \tilde{\Lambda}$. As we have seen above, $\tilde{\Lambda}$ is principal; so we may assume that $\tilde{\Lambda}=\tilde{\mathfrak{D}}$ and hence that $\alpha \tilde{\mathfrak{D}} \subsetneq \Lambda \sqsubseteq \tilde{\mathfrak{D}}$. Then $\Lambda$ defines an $\bar{D}$-submodule $\bar{\Lambda}$ of $\hat{b}$ such that the natural inclusion $\overline{\mathfrak{D}} \longrightarrow \hat{\mathrm{o}}_{\Lambda}:=\{a \in \hat{\mathrm{D}} ; a \bar{\Lambda} \subseteq \bar{\Lambda}\}$ is the identity. Thus it suffices first to classify all such $\overline{0}$-modules $\bar{\Lambda}$ and then for each such $\bar{\Lambda}$ to show that its inverse image $\Lambda$ in $\mathfrak{D}$ is either principal or is not a proper $\mathfrak{D}$-ideal. First, in cases 1) and 2) the desired result follows from the following observations: $\hat{\mathrm{D}} \cong\left(R_{m} / \alpha R_{m}\right)^{2}$ and $\overline{\mathfrak{D}}$ its diagonal, where $R_{m} / \alpha R_{m} \cong \mathbb{F}_{m}$ in case 1), and in case 2) $R_{2} / 2 R_{2} \cong C[X] /(1+X)^{2}$ and $R_{3} / 2 R_{3} \cong \mathbb{F}_{4}$.

In case 3) we see that the unique possibility for $\Lambda$ other than $a_{0}$ is the $\mathfrak{a}_{0}$-module $\{a+b i+c j+d k ; a \equiv b, c \equiv d(2)\}$. But then $\Lambda=\mathfrak{a}_{0}(1+i)$ and hence is principal as desired. Finally, consider the case 4). In this case we have $\tilde{\mathfrak{o}}=M_{2}(\boldsymbol{Z})$. First we take $\mathfrak{D}=\mathfrak{a}_{4}$. Set $\mathfrak{o}^{\prime}=\mathfrak{a}_{4}^{\prime}$ and $\overline{\mathfrak{D}}^{\prime}=\mathfrak{o}^{\prime} / 2 \tilde{\mathrm{D}}$. Then, up to right multiplication by elements of $\hat{\mathfrak{v}}$ the $\overline{\mathbf{b}}$-modules contained in $\hat{\mathbf{b}}$ are one of the following:

$$
\begin{aligned}
& \bar{\Lambda}_{1}=\left\langle\left(\begin{array}{ll}
1 & 1 \\
0 & 0
\end{array}\right)\right\rangle, \quad \bar{\Lambda}_{2}=\left\langle\left(\begin{array}{ll}
1 & 1 \\
0 & 0
\end{array}\right),\left(\begin{array}{ll}
0 & 0 \\
1 & 1
\end{array}\right)\right\rangle, \quad \bar{\Lambda}_{3}=\left\langle\left(\begin{array}{ll}
1 & 0 \\
1 & 0
\end{array}\right),\left(\begin{array}{ll}
0 & 1 \\
0 & 1
\end{array}\right)\right\rangle, \\
& \bar{\Lambda}_{4}=\overline{0}, \quad \bar{\Lambda}_{5}=\overline{\mathfrak{D}}^{\prime} .
\end{aligned}
$$

Let $\Lambda_{\mu}$ be the inverse images of $\bar{\Lambda}_{\mu}$ in $\tilde{\mathfrak{D}}$. Then we have $\Lambda_{1}=\mathfrak{o}^{\prime} \alpha_{4}$ (cf. Lemma 2.11) and hence $\Lambda_{1}$ is $\mathfrak{D}^{\prime}$-principal; $\Lambda_{2}$ is an $M_{2}(\mathbb{Z})$-module and hence it is neither proper $\mathfrak{b}$-nor $\mathfrak{b}^{\prime}$-ideal. The same is also true for $\Lambda_{3}$ since $(\alpha / 2) \Lambda_{3} \subseteq \Lambda_{3}$. This proves the desired assertion in this case. The case $\mathfrak{v}=\mathfrak{a}_{6}$ is treated similarly and will be omitted. 
Lemma 2.13. Let $(A, 0, H, M)$ be a quadruple in 2. 1) If $\operatorname{rank}_{Q} A=2$ or 4 , then $M$ is a free $\mathfrak{D}$-module except the case $\mathfrak{D}=\mathfrak{o}_{1}^{\prime}$. If $\mathfrak{D}=\mathfrak{o}_{1}^{\prime}$, then $M$ is either free or isomorphic to the direct sum $\mathfrak{o}_{1}^{\prime} \oplus \tilde{\mathfrak{o}}$ as $\mathfrak{o}_{1}^{\prime}-$ modules. 2) If $A=\mathbb{Q} \oplus \mathbb{Q}\left(e_{2 m}\right)$, and hence if $\mathfrak{0}=\mathbb{Z} \oplus R_{m}, M$ is $\mathbf{0}$-isomorphic to $\mathbb{Z}^{2} \oplus \mathfrak{D}$, where $(a, b) \in \mathfrak{0}$ with $a \in \mathbb{Z}$ and $b \in R_{m}$ acts on the $\mathbb{Z}^{2}$-factor by the multiplication by $a$.

Proof. When $\operatorname{rank}_{Q} A=4$, we have an isomorphism $w: A \rightarrow M_{Q}$ of $A$-modules. Let $A=w^{-1}(M)$. Then by $\left.Q 3\right) A$ is a proper 0 -ideal of $A$, and hence is free by Lemma 2.12. It follows that $M$ also is free. If $\operatorname{rank}_{Q} A=2$ and $A$ is a field, then $\mathfrak{D}$ is a principal ideal domain by Lemma 2.12 so that $M$ is again free. The remaining cases are: 1) $A=\mathbb{Q}^{2}, \mathfrak{o}=\tilde{\mathfrak{D}}, \mathfrak{o}_{1}^{\prime}$, and 2) $A=\mathbb{Q} \oplus \mathbb{Q}\left(e_{2 m}\right)$.

Let $\tilde{\mathfrak{d}}$ be the maximal order of $A$. Then $\tilde{\mathfrak{D}}$ is the direct sum $\tilde{D}=\mathfrak{o}_{1} \oplus \mathfrak{o}_{2}$, where $\mathfrak{o}_{i}$ is the maximal order of the $i$-th factor of $A$. Accordingly, $\tilde{M}:=\tilde{D} M\left(\subseteq M_{Q}\right)$ becomes a direct sum $\tilde{M}=M_{1} \oplus M_{2}$, where $M_{i} \cong \mathbb{Z}^{2}$ if $\mathfrak{o}_{i} \cong \mathscr{Z}$ and $M_{i} \cong \mathfrak{o}_{i}$ otherwise. Hence if $\mathfrak{o}=\tilde{\mathfrak{D}}, \tilde{M}$ is just as claimed. The cases $\mathfrak{D} \neq \tilde{\mathfrak{D}}$, namely the cases $\mathfrak{D}=\mathfrak{D}_{1}^{\prime}$ or $\hat{\mathfrak{D}}_{m}$, can be treated analogously as in the proof of the previous lemma in the case of non-maximal orders; so we leave the detail to the reader.

Summarizing what we have proved so far, we get the following:

Proposition 2.14. Under the previous notations any quadruple $(A, \mathfrak{D}$, $H, M)$ with $\operatorname{rank}_{Q} A \leqq 4$ in 2 is isomorphic to one of the quadruples listed in the following table.

Table 1

\begin{tabular}{|c|c|c|c|c|}
\hline No. & $A$ & D & $H$ & $M$ \\
\hline 1 & $\begin{array}{c}\left(1 \leqq\left(e_{2 m}\right)\right. \\
(1 \leqq m \leqq 6)\end{array}$ & $\tilde{\mathfrak{D}}$ & $\tilde{\mathfrak{D}}_{f}^{\mathrm{x}} \cong \mathfrak{C}_{2 m}$ & $\begin{array}{c}\mathfrak{0}^{S} \\
\left(s=4 / \mathrm{rank}_{Q} A\right)\end{array}$ \\
\hline $\begin{array}{l}2 \\
2^{\prime}\end{array}$ & $Q \oplus \mathbb{Q}$ & $\begin{array}{l}\tilde{\mathfrak{D}} \\
\mathfrak{o}_{1}^{\prime}\end{array}$ & $\tilde{D}^{\times} \cong \mathfrak{c}_{2} \times \mathfrak{c}_{2}$ & $\begin{array}{c}\mathfrak{o}^{2} \\
\mathfrak{o}^{2}, \mathfrak{o} \oplus \tilde{\mathfrak{d}}\end{array}$ \\
\hline 3 & $Q \oplus Q(i)$ & $\tilde{\mathfrak{D}}_{2} \hat{\mathfrak{o}}_{2}$ & $\tilde{\mathfrak{D}}^{\times} \cong \mathfrak{C}_{2} \times \mathfrak{C}_{4}$ & $\mathbb{Z}^{2} \oplus \mathbb{0}$ \\
\hline
\end{tabular}




\begin{tabular}{|c|c|c|c|c|}
\hline 4 & \multirow{2}{*}{$\boldsymbol{Q} \oplus \boldsymbol{Q}(\boldsymbol{\rho})$} & $\tilde{\mathfrak{o}}$ & $\tilde{\mathfrak{D}}^{\times} \cong \mathfrak{E}_{2} \times \mathfrak{E}_{6}$ & \multirow{2}{*}{$\mathbb{Z}^{2} \oplus \mathbb{0}$} \\
\hline 5 & & $\tilde{\mathfrak{\mathfrak { b }}}, \hat{\mathfrak{o}}_{3}$ & $\hat{\mathfrak{D}}_{3}^{\times} \cong \mathfrak{S}_{6}$ & \\
\hline 6 & \multirow{2}{*}{$\boldsymbol{Q}(i) \oplus \boldsymbol{Q}(i)$} & $\tilde{\mathfrak{v}}, \mathfrak{o}_{2}$ & $\tilde{\mathfrak{D}}^{\times} \cong \mathfrak{E}_{4} \times \mathfrak{C}_{4}$ & \multirow{2}{*}{$\mathfrak{D}$} \\
\hline 7 & & $\tilde{\mathfrak{D}}, \mathfrak{o}_{2}, \mathfrak{o}_{2}^{\prime}$ & $\mathfrak{D}_{2}^{\prime \times} \cong \mathfrak{夭}_{2} \times \mathfrak{夭}_{4}$ & \\
\hline 8 & \multirow{3}{*}{$\boldsymbol{Q}(\rho) \oplus \boldsymbol{Q}(\rho)$} & $\tilde{\mathfrak{D}}$ & $\tilde{\mathfrak{D}}^{\times} \cong \mathfrak{V}_{6} \times \mathfrak{E}_{6}$ & \multirow{3}{*}{0} \\
\hline 9 & & $\tilde{\mathfrak{D}}, \mathfrak{o}_{3}$ & $\mathfrak{D}_{3}^{\times} \cong \mathfrak{E}_{3} \times \mathfrak{E}_{6}$ & \\
\hline 10 & & $\tilde{\mathfrak{o}}, \mathfrak{o}_{3}^{\prime}$ & $\mathfrak{D}_{3}^{\prime \times} \cong \mathfrak{E}_{2} \times \mathfrak{G}_{6}$ & \\
\hline 11 & $\boldsymbol{Q}(i) \oplus \boldsymbol{Q}(\rho)$ & $\tilde{\mathfrak{o}}$ & $\tilde{\mathfrak{D}}^{\times} \cong \mathfrak{E}_{4} \times \mathfrak{F}_{6}$ & 0 \\
\hline 12 & \multirow[b]{3}{*}{$F^{\prime}$} & $\mathfrak{a}_{,} \mathfrak{a}_{0}$ & $\mathfrak{a}_{0}^{\times} \cong \mathscr{Q}$ & \multirow{5}{*}{$\mathfrak{v}$} \\
\hline 13 & & $\mathfrak{a}$ & $\mathfrak{a}^{\times} \cong \mathfrak{T}$ & \\
\hline 14 & & $\mathfrak{b}$ & $\mathfrak{b}^{\times} \cong \mathfrak{D}$ & \\
\hline 15 & \multirow{2}{*}{$M_{2}(Q)$} & $M_{2}(\mathbb{Z}), \mathfrak{a}_{4}, \mathfrak{a}_{4}^{\prime}$ & $\hat{D}_{4} \cong \mathscr{D}_{4}$ & \\
\hline 16 & & $M_{2}(\mathbb{Z}), \mathfrak{a}_{6}$ & $\hat{D}_{6} \cong \mathfrak{D}_{6}$ & \\
\hline
\end{tabular}

We note that by Lemma 2.1 the table is also considered as classifying the rational representation types of pairs $(T, G)$ with $-1 \in G$, under the assumption that $\operatorname{rank}_{Q} A \leqq 4$.

2.5. We next study the structure of the normalizer $N=N_{F}$ of any quadruple $F=(A, \mathfrak{o}, H, M)$ in 2 . For any such $F$ we denote by $\bar{N}_{F}$ the group of algebra automorphisms of $A$ which leave $D$ and $H$ invariant. First we determine the structure of $\bar{N}_{F}$.

Proposition 2.15. Let $G_{m}$ be the Galois group of $\mathbb{Q}\left(e_{2 m}\right)$ over $\mathbb{Q}$. Let $\Delta_{m}$ be the diagonal of $G_{m} \times G_{m}$. Let $\tau_{m}$ be the involution of $Q\left(e_{2 m}\right)^{2}$ which interchanges the two factors. Then for each quadruple $F$ in Table 1 the corresponding group $\bar{N}_{F}$ defined above is given in the following table. 
Table 2

\begin{tabular}{|c|c|}
\hline No. of $F$ in Table 1 & $\bar{N}_{F}$ \\
\hline 1 & $G_{m} \cong \mathfrak{夭}_{\varphi(2 m)}$ \\
\hline $2,2^{\prime}$ & $\left\langle\tau_{1}\right\rangle \cong \mathfrak{c}_{2}$ \\
\hline $3-5$ & $\{e\} \times G_{m} \cong \mathfrak{C}_{2}$ \\
\hline $6-9,10$ with $\mathfrak{v} \neq \mathfrak{p}_{3}^{\prime}$ & $\left(G_{m} \times G_{m}\right) \rtimes\langle\tau\rangle \cong \mathfrak{D}_{4}$ \\
\hline 10 with $\mathrm{D}=\mathrm{o}_{3}^{\prime}$ & $\Delta_{3} \rtimes\left\langle\tau_{3}\right\rangle \cong \mathfrak{D}_{2} \cong \mathfrak{S}_{2} \times \mathfrak{E}_{2}$ \\
\hline 11 & $G_{m} \times G_{m} \cong \mathfrak{E}_{2} \times \mathfrak{E}_{2}$ \\
\hline 12,13 & $\left\langle a^{\times},(1+i) / \sqrt{2}\right\rangle /\{ \pm 1\} \cong \bigcirc /\{ \pm 1\} \cong \widetilde{S}_{4}$ \\
\hline 14 & $\left\langle\mathfrak{b}^{\times},(\sqrt{3}+k) / 2\right\rangle /\{ \pm 1\} \cong \hat{\mathfrak{S}} /\{ \pm 1\} \cong \mathfrak{D}_{6}$ \\
\hline $\mathfrak{v}=M_{2}(\mathbb{Z})$ & $\hat{D}_{4} /\{ \pm 1\} \cong \mathfrak{S}_{2}$ \\
\hline $\mathfrak{D}=\mathfrak{a}_{4}, \mathfrak{a}_{4}^{\prime}$ & $\left\langle\hat{D}_{4}, \alpha_{4} / \sqrt{2}\right\rangle /\{ \pm 1\} \cong \mathfrak{D}_{4}$ \\
\hline $\mathfrak{o}=M_{2}(\mathbb{Z})$ & $\hat{D}_{6} /\{ \pm 1\} \cong \mathfrak{S}_{3}$ \\
\hline $\mathfrak{v}=\mathfrak{a}_{6}$ & $\left\langle\hat{D}_{6}, \alpha_{6} / \sqrt{3}\right\rangle /\{ \pm 1\} \cong \mathfrak{S}_{6}$ \\
\hline
\end{tabular}

In Nos. 12-16 the elements act on $A$ by inner automorphisms.

Proof. Except for 12-16 the assertion is almost trivial. For 12-14 see Lemma 2.8 and (5). For 15 and 16 the result follows from Lemma 2.11 in view of the fact that $\alpha_{4}$ (resp. $\left.\alpha_{6}\right)$ normalizes $a_{4}, a_{4}^{\prime}$ (resp. $a_{6}$ ), but not $M_{2}(\mathbb{Z})$.

Let $N=N_{F}$ and $\bar{N}=\bar{N}_{F}$ be as above. We then have the natural homomorphism $f: N \rightarrow \bar{N}$, whose kernel is identified with Auto $M$, the group of $\mathrm{p}$-linear automorphisms of $M$. Suppose first that $M$ is a free $\mathfrak{D}$-module $\mathfrak{D}^{s}, s \geqq 1$. Auto $M \cong \mathrm{GL}_{s}(\mathfrak{o})^{*}$ and we can define the $\mathbb{Z}$ linear action of $\bar{N}$ on $M$ by

$$
h\left(a_{1}, \ldots, a_{s}\right)=\left(h\left(a_{1}\right), \ldots, h\left(a_{s}\right)\right), h \in \bar{N}, a_{i} \in \mathfrak{D} ;
$$

then for $\alpha \in \mathrm{o}$ we have $h\left(\alpha h^{-1}\left(a_{i}\right)\right)=h(\alpha) a_{i}$. Hence we get the natural embedding $\bar{N} \longrightarrow N$ and the semidirect product decomposition

$$
N \cong \mathrm{Aut}_{\mathrm{p}} M \rtimes \overline{\mathrm{N}} \text {. }
$$

*) In the noncommutative case we always have $s=1$, and $\mathrm{GL}_{1}(\mathrm{D})=\mathrm{D}^{*}$ acts on $M=\mathrm{D}$ by right multiplication. 
Next, in the case $(A, \mathfrak{o}, M)=\left(Q^{2}, \mathfrak{o}_{1}^{\prime}, \mathfrak{o}_{1}^{\prime} \oplus \tilde{\mathfrak{D}}\right)$ we have the natural embedding $M \longrightarrow \tilde{\mathfrak{o}}^{2}$, and the automorphism of $\tilde{\mathfrak{o}}^{2}$ defined by (10) leaves $M$ invariant. Hence we have again the natural embedding $\bar{N} \longrightarrow$ Aut $_{z} M$ and (11) holds in this case also. Finally in the case $(A, \mathfrak{D}, M)=$ $\left(\boldsymbol{Q} \oplus \boldsymbol{Q}\left(e_{2 m}\right), \boldsymbol{Z} \oplus \mathrm{D}\right) \quad$ (cf. Lemma 2.13) we get the natural embedding $M=Z^{2} \oplus 0 \longrightarrow Z^{2} \oplus R_{m}$ and the action of $\bar{N}:=G_{m}$ on $\mathbb{Z}^{2} \oplus R_{m}$ defined by $h(a, b)=(a, h b), a \in \mathbb{Z}^{2}, b \in R_{m}, h \in G_{m}$, leaves $\mathbb{Z}^{2} \oplus \mathbb{0}$ invariant. Hence in this case also we have the natural embedding $\bar{N} \longrightarrow$ Aut $_{z} M$ and (11) holds true. Let $\hat{\Gamma}_{0}(m)=\left\{\left(\begin{array}{ll}a & b \\ c & d\end{array}\right) \in \mathrm{GL}_{2}(\boldsymbol{Z}) ; a \equiv 1, c \equiv 0(m)\right\}$, where $m=2,3$. Then we easily obtain the following:

Lemma 2.16. Let $F$ be any quadruple in 2 with $\operatorname{rank}_{Q} A \leqq 4$. Let $N=N_{F}$ and $\bar{N}=\bar{N}_{F}$ be as above. Then there exists a natural embedding $\bar{N} \longrightarrow N$ and with respect to this embedding $N$ is naturally a semidirect product $N=\mathrm{Aut}_{\mathrm{D}} M \rtimes \bar{N}$. Here the structure of $\mathrm{Aut}_{0} M$ is up to isomorphisms given as follows; 1) $\mathrm{GL}_{s}(\mathfrak{b})$ if $\left.M \cong \mathfrak{o}^{s}, 2\right) \hat{\Gamma}_{0}(2)$ if $(\mathfrak{o}, M)=\left(\mathfrak{o}^{\prime}, \mathfrak{o}^{\prime} \oplus \mathfrak{o}\right)$, 3a) $\mathrm{GL}_{2}(\boldsymbol{Z}) \times R_{m}^{\times}$if $(A, \mathfrak{o})=\left(\boldsymbol{Q} \oplus \boldsymbol{Q}\left(e_{2 m}\right)\right.$, ̃) and $\left.3 \mathrm{~b}\right) \hat{\Gamma}_{0}(m) \times R_{m}^{\times}$if $(A, \mathfrak{o})=\left(\boldsymbol{Q} \oplus \boldsymbol{Q}\left(e_{2 m}\right), \hat{\mathfrak{o}}_{m}\right)$.

2.6. Finally we shall determine the structure of $W_{F}$ for each $F$ in Table 1. First we introduce some notations. We denote by $\mathscr{S}$ the upper half plane; $\mathfrak{S}=\{z ; \operatorname{Im} z>0\}$, and by $\mathbb{P}^{1}$ the complex projective line. $\{*\}$ will denote the space consisting of a single point. For $\mathbb{K}=\boldsymbol{R}$ or $\boldsymbol{C}$ we set

$$
C_{\boldsymbol{K}}=\left\{\left(\begin{array}{rr}
a & b \\
-b & c
\end{array}\right) \in M_{2}(\boldsymbol{K}) ; a^{2}+b c=-1\right\} .
$$

$\mathrm{GL}_{2}(\boldsymbol{K})$ acts transitively on the set $C_{\boldsymbol{K}}$ by inner automorphisms with stabilizer at $[1,-1]$ (resp. $(i,-i))$ (cf. Notations) given by $\left\{\left(\begin{array}{rr}a & b \\ -b & a\end{array}\right)\right.$; $a, b \in \boldsymbol{R}\} \cong \boldsymbol{C}^{*}\left(\right.$ resp. $\left.\left\{(a, b) ; a, b \in \boldsymbol{C}^{*}\right\} \cong \boldsymbol{C}^{*} \times \boldsymbol{C}^{*}\right)$ if $\boldsymbol{K}=\boldsymbol{R}$ (resp. $\boldsymbol{C}$ ). Thus $C_{\boldsymbol{R}} \cong \mathrm{GL}_{2}(\boldsymbol{R}) / \boldsymbol{C}^{*} \cong \tilde{\mathfrak{E}}$ and $C_{\boldsymbol{C}} \cong \mathrm{GL}_{2}(\boldsymbol{C}) / \boldsymbol{C}^{* 2}$, where $\tilde{\mathfrak{E}}=\mathfrak{S} \times \overline{\mathfrak{E}}$ with $\overline{\mathcal{S}}$ the lower half plane.

On the other hand, let $X$ be the subset of the pure quaternions defined by

$$
X=\left\{q \in H ; q^{2}=-1\right\}=\left\{a i+b j+c k ; a^{2}+b^{2}+c^{2}=1\right\} .
$$

Then $\boldsymbol{H}^{\times}$acts transitively on the set $X$ by inner automorphisms with stabilizer at $i$ given by $\left\{a+b i \in \boldsymbol{H}^{\times}\right\} \cong \boldsymbol{C}^{*}$. Thus we have the natural 
identifications

$$
X=\mathbb{H}^{\times} / \mathbb{C}^{*}=\mathbb{P}^{1} \text { 。 }
$$

Now noting that $W_{F}$ depends only on the pair $\left(A_{R}, M_{R}\right)$, we obtain the following:

Proposition 2.17. In the above notations for each quadruple $F=$ $(A, D, H, M)$ in Table 1 the corresponding complex manifold $W_{F}$ is given in the following table. In particular each connected component of $W_{F}$ is naturally a homogeneous complex manifold.

Table 3 .

\begin{tabular}{l|c|c|c|l}
\hline $\begin{array}{c}\text { No. of } F \text { in } \\
\text { Table 1 }\end{array}$ & $A_{R}$ & $M_{R}$ & $W_{F}$ & \multicolumn{1}{|c}{ structure } \\
\hline \multicolumn{1}{c|}{$(m=1)$} & $\mathbb{R}$ & $\mathbb{R}^{4}$ & $W$ & \\
$1(m=2,3)$ & $\mathbb{C}$ & $\mathbb{C}^{2}$ & $C_{C} \Perp\{ \pm(i, i)\}$ & $\mathrm{GL}_{2}(\mathbb{C}) / \mathbb{C}^{* 2} \Perp\left\{^{*}\right\} \Perp\left\{^{*}\right\}$ \\
$(m=4,5,6)$ & $\mathbb{C}^{2}$ & $\mathbb{C}^{2}$ & $\{( \pm i, \pm i)\}$ & 4 points \\
$2,2^{\prime}$ & $\mathbb{R}^{4}$ & $\mathbb{R}^{4}$ & $C_{R} \times C_{R}$ & $\tilde{\mathfrak{Q}} \times \tilde{\mathfrak{S}}$ \\
$3-5$ & $\mathbb{R} \oplus \mathbb{C}$ & $\mathbb{R}^{2} \oplus \mathbb{C}$ & $C_{R} \Perp C_{R}$ & $\tilde{\mathfrak{Q}} \Perp \tilde{\mathfrak{S}}$ \\
$6-11$ & $\mathbb{C}^{2}$ & $\mathbb{C}^{2}$ & $\{( \pm i, \pm i)\}$ & 4 points \\
$12-14$ & $\mathbb{H}$ & $\mathbb{H}$ & $X$ & $\mathbb{P}$ \\
$15-16$ & $M_{2}(\mathbb{R})$ & $M_{2}(\mathbb{R})$ & $C_{R}$ & $\tilde{\mathfrak{C}}$ \\
\hline
\end{tabular}

Remark 2. 18. As a complex manifold, $\mathrm{GL}_{2}(\mathbb{C}) / \mathbb{C}^{* 2} \cong \mathrm{SL}_{2}\left(\mathbb{C}^{*}\right) / \mathbb{C}^{*}$ here is isomorphic to the total space of the holomorphic tangent bundle over the complex projective line (cf. [19], Th.4.5). In particular the complex structure is not the one induced from the complex Lie group structure of $\mathrm{GL}_{2}(\mathbb{C})$.

Proof. Let $L$ be the closed Lie subgroup $\mathrm{Aut}_{A_{R}} M_{R}$ of $\mathrm{Aut}_{R} M_{R} \cong$ $\mathrm{GL}_{4}(\mathbb{R})$ consisting of $A_{R}$-linear automorphisms。 ( $L$ also is the centralizer of $H$ in Aut $_{R} M_{R}$ o) Then we have $W_{F}=\left\{x \in L ; x^{2}=-1\right\}$, and $L$ operates on $W_{F}$ via inner automorphisms; the orbits are just the conjugacy classes $C_{i}$ in $W_{F}$. Thus if we choose a representative $x_{i}$ for each $C_{i}, C_{i}$ is considered a homogeneous complex manifold $L / L_{i}$ where $L_{i}$ is the centralizer of $x_{i}$. Indeed, according to the numbering of Table 1 the triples $\left(A_{R}, L, x_{i}, 1 \leqq i \leqq k\right)$ for suitable $x_{i}$ are given 
as follows. 1. $\left(\boldsymbol{R}, \mathrm{GL}_{4}(\boldsymbol{R}),\left(\begin{array}{rr}0_{2} & 1_{2} \\ -1_{2} & 0_{2}\end{array}\right)\right)$ if $m=1,\left(\boldsymbol{C}, \mathrm{GL}_{2}(\boldsymbol{C}),(i,-i)\right.$, $\pm(i, i))$ if $m=2,3$ and $\left(\boldsymbol{C} \oplus \boldsymbol{C}, \boldsymbol{C}^{*} \times \boldsymbol{C}^{*},( \pm i, \pm i)\right)$ if $m=4,5,6$. 2, 2'. $\left.\left(\boldsymbol{R} \oplus \boldsymbol{R}, \mathrm{GL}_{2}(\boldsymbol{R}) \times \mathrm{GL}_{2}(\boldsymbol{R}), \pm[1,-1]\right)\right), 3-5 .\left(\boldsymbol{R} \oplus \boldsymbol{C}, \mathrm{GL}_{2}(\boldsymbol{R}) \times \boldsymbol{C}^{*}\right.$, $( \pm[1,-1], \pm i)), 6-11$. $\left(\boldsymbol{C} \oplus \boldsymbol{C}, \boldsymbol{C}^{*} \times \boldsymbol{C}^{*},( \pm i, \pm i)\right), 12-14 .\left(\boldsymbol{H}, \boldsymbol{H}^{\times}, i\right)$, and finally, 15-16. $\left(M_{2}(\boldsymbol{R}), \mathrm{GL}_{2}(\boldsymbol{R}), \pm[1,-1]\right)$. The lemma follows from this immediately.

2.7. Let $H$ be any finite subgroup of $\mathrm{GL}_{4}(\mathbb{Z})$ such that $\mathrm{W}_{H} \neq \emptyset$ (cf. 2.1). Let $(H)$ be the conjugacy class of $H$ in $\mathrm{GL}_{4}(\mathbb{Z})$. Let $\mathscr{M}_{(H)}$ be the moduli space of the pairs $(T, G)$ with fixed rational representation type $(H)$. Let $F=F_{H}=(A, \mathfrak{D}, H, M)$ be the quadruple corresponding to $H$ by Lemma 2. 1. Let $N_{F}$ and $W_{F}$ be the normalizer of $F$ and the set of $A_{R}$-linear complex structures on $M_{R}$ respectively. Then we have $\mathscr{M}_{(H)}=N_{F} \backslash W_{F}$ by Lemma 2.1. Since we have determined $N_{F}$ and $W_{F}$ in Proposition 2. 15, Lemma 2.16 and Proposition 2. 17 when $\operatorname{rank}_{Q} A \leqq 4$, the structure of $\mathscr{M}_{(H)}$ has essentially been determined in this case already. However we find the following slightly modified presentation more convenient. Namely we choose one representative from each $N_{F}$-equivalence class of connected components of $W_{F}$ 。 Let $W_{\nu}, \nu=1, \ldots, s$, be any such representatives. (Actually $s=1$ or 2.) Let $N_{\nu}$ be the stabilizer of $W_{\nu}$ in $N_{F}$. Then the following is also a canonical presentation of $\mathscr{M}_{(H)}$ as a union of locally homogeneous spaces;

$$
\mathscr{M}_{(H)}=\Perp_{\nu} N_{\nu} \backslash W_{\nu} .
$$

Let $H$ be the centralizer of $W_{F}$ in Aut $_{Z} M$, i. e., $H=\left\{g \in \mathrm{Aut}_{\boldsymbol{Z}} M ; g J=J g\right.$ for any $J \in W_{F}$. Then $H$ is a normal subgroup of $N_{F}$ containing $H$ and the action of $N_{F}$ on $W_{F}$ factors through the quotient $N_{F} / \hat{H}$. Hence in the above presentation (13) we may replace $N_{\nu}$ freely by $\bar{N}_{\nu}:=N_{\nu} / H_{\nu}$ for any normal subgroup $H_{\nu}$ of $N_{\nu}$ contained in $\hat{H}$, a typical example being given by $H_{\nu}=H \cap N_{\nu}$. Now we shall describe the structure of $\mathscr{M}_{(H)}$ in the sense of (13) modulo the remark just made.

Proposition 2.19. Let $H, F=(A, \mathfrak{D}, H, M)$, and $\mathscr{M}_{(H)}$ be as above. Suppose that $\operatorname{rank}_{Q} A \leqq 4$. Then according to the classification of $F$ in Table 1, we have the structure of $\mathscr{M}_{(H)}$ as a union of locally homogeneous spaces as in the following table. 
Table 4.

\begin{tabular}{|c|c|c|}
\hline \multicolumn{2}{|c|}{ No. } & $\mathscr{M}_{(H)}$ \\
\hline la & $m=1$ & $\mathrm{SL}_{4}(\mathbb{Z}) \backslash W^{+}$ \\
\hline $1 \mathrm{~b}$ & $m=2,3$ & $\left(\mathrm{GL}_{2}\left(R_{m}\right) \backslash \mathrm{GL}_{2}(\mathbb{C}) /\left(\mathbb{C}^{*}\right)^{2}\right) / \mathbb{C}_{2} \Perp\{*\}$ \\
\hline 1c & $m=4,6$ & $\{*\} \Perp\{*\}$ \\
\hline $1 d$ & $m=5$ & $\{*\}$ \\
\hline 2 & & $\operatorname{sym}^{2}\left(\mathrm{SL}_{2}(\mathbb{Z}) \backslash H\right) \cong \mathbb{C}$ \\
\hline$?^{\prime}$ & $M=\mathfrak{D}^{2}$ & $\operatorname{sym}^{2}(\Gamma(2) \backslash H) / \widetilde{S}_{3} \cong\left(\mathbb{C}^{*} \times \mathbb{C}^{*}\right) / \mathbb{S}_{3}$ (rational) \\
\hline 2 & $M=\mathfrak{o} \oplus \tilde{\mathfrak{D}}$ & $\operatorname{sym}^{2}\left(\Gamma_{0}(2) \backslash H\right) \cong \mathbb{C} \times \mathbb{C}^{*}$ \\
\hline $3-5$ & $\mathfrak{D}=\tilde{\mathfrak{D}}$ & $\mathrm{SL}_{2}(\mathbb{Z}) \backslash H \cong \mathbb{C}$ \\
\hline - & $\mathfrak{D}=\mathfrak{\mathfrak { o }}_{m}$ & $\Gamma_{0}(m) \backslash H \cong \mathbb{C}^{*}$ \\
\hline $6-9,11$ & & {$[*]$} \\
\hline $10 \mathrm{a}$ & $\mathfrak{D} \neq \mathfrak{D}_{3}^{\prime}$ & {$["]$} \\
\hline $10 \mathrm{~b}$ & $\mathfrak{D}=\mathfrak{D}_{3}^{\prime}$ & $\{*\} \Perp\{*\}$ \\
\hline 12,13 & & $\mathfrak{S}_{4} \backslash \mathbb{P}^{1} \cong \mathbb{P}^{1}$ \\
\hline 14 & & $\mathfrak{D}_{6} \backslash \mathbb{P}^{1} \cong \mathbb{P}^{1}$ \\
\hline 15.16 & $\mathfrak{v}=M_{2}(\mathbb{Z})$ & $\mathrm{SL}_{2}(\mathbb{Z}) \backslash H \cong \mathbb{C}$ \\
\hline 10,10 & $\mathfrak{o}=\mathfrak{a}_{4}, \mathfrak{a}_{4}^{\prime}, \mathfrak{a}_{6}$ & $\left(\check{\mathfrak{o}}_{1}^{\times} \rtimes\left(\mathfrak{S}_{2}\right) \backslash H \cong \mathbb{C}-\{\right.$ points $\}$ \\
\hline
\end{tabular}

Here $W^{+}$is a connected component of $W$, and $\operatorname{sym}^{2}(X)$ denotes the symmetric product of $X$. Further $\Gamma_{0}(m)=\left\{\left(\begin{array}{ll}a & b \\ c & d\end{array}\right) \in \mathrm{SL}_{2}(\mathbb{Z}) ; c \equiv\right.$ $0(m)\}$. In $1 \mathrm{~b}$ the generator of $\mathbb{E}_{2}$ acts on $\mathrm{GL}_{2}(\mathbb{C})$ via the complex conjugation, leaving invariant the subgroups $\mathrm{GL}_{2}(\mathfrak{D})$ and $\mathbb{C}^{* 2}$. On the other hand, consider $\mathfrak{D}$ and $\tilde{\mathfrak{D}}$ as subgroups of $\mathbb{H}^{\times}$by (5). Then we have the induced action of $\Im_{4} \cong \mathfrak{D} /\{ \pm 1\}$ and $D_{6} \cong \mathfrak{D} /\{ \pm 1\}$ on $\mathbb{P} \cong \mathbb{H}^{\times} / \mathbb{C}^{*}$. These are the actions in $12-14$. In the last case $\hat{\mathrm{o}}_{1}^{\times}:=\mathrm{SL}_{2}(\mathbb{Z}) \cap \mathfrak{D}^{\times} \cong \mathrm{GL}_{2}(\mathbb{Z})$ and the action of $\mathfrak{夭}_{2}=\left\langle\alpha_{k}^{\prime}\right\rangle$ is induced by the inner automorphism of $\alpha_{k}, k=4,6$.

From (8) we see that the structure of $\check{\mathfrak{o}}_{1}^{\times}$is described as follows. Let $m=k / 2$. Let $\mu_{m}: \mathrm{SL}_{2}(\mathbb{Z}) \rightarrow \mathrm{SL}_{2}(\mathbb{Z} / m \mathbb{Z})$ be the natural homomorphism. Let $H_{m}$ be the subgroup of $\mathrm{SL}_{2}(\mathbb{Z} / m \mathbb{Z})$ defined by: $H_{m}=$ 
$\langle[1,1]\rangle \cong \mathfrak{\Im}_{2}$ if $k=4$, and $=\left\langle\left(\begin{array}{rr}0 & -1 \\ 1 & 1\end{array}\right)\right\rangle \cong \mathfrak{\Im}_{6}$ if $k=6$. Then

$$
\check{\mathfrak{o}}_{1}^{\times}=\mu_{m}^{-1}\left(H_{m}\right) \text {. }
$$

Proof. Let $N=N_{F}$ and $\bar{N}=\bar{N}_{F}$. We may identify $M_{R}$ with $A_{R}$ when $M=0$. As a typical case, we first consider $1 \mathrm{c}$ and $1 \mathrm{~d}$. By Proposition 2.15 and Lemma 2.16 we have $N=G_{m} \ltimes \tilde{\mathfrak{b}}^{\times}$in this case. Let the action of $N$ on $A$ extend to the $\mathbb{R}$-linear action on $A_{R}$. Fix an $\mathbb{R}$-algebra isomorphism $A_{R} \cong \mathbb{C}^{2}$. The problem is to identify the action of $N$ on the set $C:=\left\{J_{\varepsilon \eta}:=(\varepsilon i, \eta i) ; \varepsilon= \pm 1, \eta= \pm 1\right\}$ of complex structures on $C^{2}=\mathbb{R}^{4}$. First of all, the action of $\tilde{D}^{\times}$is induced by the multiplication of the elements of $C^{* 2}$ via the natural inclusion $\tilde{D}^{\times} \longrightarrow$ $A_{R}^{\times} \cong C^{* 2}$; hence $\tilde{\mathfrak{D}}^{\times}$acts on $C$ trivially and the action of $N$ factors through $G_{m}$. Since $G_{m}$ acts on $A_{R}$ as $\mathbb{R}$-algebra automorphisms, we have the natural inclusion $G_{m} \longrightarrow$ Aut $_{R} A_{R} \cong$ Aut $_{R} C^{2} \cong\left\langle\iota_{1}, \iota_{2}\right\rangle \rtimes\langle\tau\rangle \cong \mathfrak{I}_{4}$, where $\iota_{\alpha}$ is the complex conjugation on the $\alpha$-th factor and $\tau$ is the interchanging of the two factors. Now $G_{m} \cong \mathfrak{F}_{2} \times \mathfrak{F}_{2}$ if $m=4$ or 6 and $\cong \mathfrak{F}_{4}$ if $m=5$. Hence we must have $G_{m}=\left\langle\iota_{1} \ell_{2}, \tau\right\rangle$ if $m=4,6$ (note that $A_{R}^{G_{m}} \cong \boldsymbol{R}$ ) and $=\left\langle\iota_{1} \tau\right\rangle$ if $m=5$. The action of $\iota_{1} \iota_{2}, \tau, \iota_{1} \tau$ on $C$ are easily identified; $\ell_{1} \iota_{2}\left(J_{\varepsilon \eta}\right)=J_{-\varepsilon_{1}-\eta}, \tau\left(J_{\varepsilon \eta}\right)=J_{\eta \varepsilon}$ and $\iota_{1} \tau\left(J_{\varepsilon \eta}\right)=J_{\eta_{1}-\varepsilon}$. It follows that $G_{m}$ acts transitively on $C$ if $m=5$ and there exist two $G_{m}$-orbits $\{ \pm(i, i)\}$ and $\{ \pm(i,-i)\}$ if $m=4,6$ 。 This verifies Table 4 in this case. No.6-10 can be treated similarly.

For $1 \mathrm{~b}$ we only note that $G_{m}$ identifies $\pm(i, i)$ (cf. Proposition 2. 17). (No. 1, $m=1$, is trivial.) The result for 2-5 follows readily from the fact that $(1,-1) \in \mathrm{GL}_{2}(\mathbb{R})$ interchanges $\mathscr{S}$ and $\overline{\mathscr{S}}$ in $\tilde{\mathscr{E}}$, and $(1,-1) \in \hat{\Gamma}_{0}(m)$. (We also note that $\left(\mathrm{SL}_{2}(\mathbb{Z}) \cap \hat{\Gamma}_{0}(3)\right) \times\langle-1\rangle$ $\cong \Gamma_{0}(3)$.)

So we consider the case where $A$ is a quaternion algebra. In this case we have $N=\mathfrak{v}^{\times} \rtimes \bar{N}$. The action of $N$ on $W_{F}$ factors through $N^{\prime}:=N / H$. First, assume that $A$ is definite. Let $K=\mathfrak{O}$ (resp. $\tilde{\text { D) }}$ if $H \cong \mathfrak{Q}$ or $\mathfrak{T}$ (resp. $\mathfrak{D})$. Let $\Gamma \subseteq \mathfrak{o}^{\times} \rtimes \bar{N}$ be the graph of the composite of the natural homomorphisms $\mathfrak{D}^{\times} \cong H \longrightarrow K \rightarrow K /\{ \pm 1\} \cong \bar{N}$. Then $\Gamma$ is easily seen to coincide with $H \subseteq N$ ( $H$ acts on $\mathbb{H}$ by $\left.\left(h x h^{-1}\right) \circ h, h \in H, x \in \mathbb{H}\right)$, and we have another semidirect product decomposition $N=\Gamma \rtimes \bar{N}$. This implies that $N^{\prime} \cong \bar{N} \cong K /\{ \pm 1\}$ naturally. Next, assume that $A$ is 
isomorphic to $M_{2}(Q)$. Then the natural image of $H$ in $\bar{N}=N / 0^{\times}$is just $\hat{D}_{k} /\{ \pm 1\}$. Then $\left\langle\alpha_{k}^{\prime}\right\rangle \ltimes \mathfrak{D}^{\times} \cong \mathfrak{F}_{2} \ltimes \mathfrak{Q}^{\times}$must be mapped surjectively onto $N / H$. Hence in $N \backslash W_{F}$ we may replace $N$ by $\left\langle\alpha_{k}^{\prime}\right\rangle \times \mathfrak{v}^{\times}$. The assertion then follows from the fact that $[1,1]$ interchanges $\mathfrak{S}$ and $\overline{\mathcal{S}}$ in $\tilde{\mathfrak{S}}$ and $[1,1] \in \hat{D}_{k} \subseteq a_{k}^{\times}$.

2. 8. Let $(T, G)$ be any pair. Let $f: G \rightarrow G L(E)$ be the complex representation of $(T, G)$. Let $u: E \rightarrow \mathbb{C}^{2}$ be any $\mathbb{C}$-linear isomorphism. Then uf maps $G$ onto a subgroup $K$ of $\mathrm{GL}_{2}(\mathbb{C})$ whose conjugacy class $(K)$ is independent of $u$ as above and depends only on $(T, G)$. is called the complex representation type of $(T, G)$.

Let $F=(A, \mathfrak{0}, H, M)$ be a quadruple in $\mathscr{Q}$ with $\operatorname{rank}_{Q} A \leqq 4$. As the proof of Proposition 2.17 shows, complex structures on $M_{R}$ from one and the same connected component of $W_{F}$ are conjugate in Aut $_{A_{R}} M_{R}$. From this we conclude that the complex representation type $(K)$ is constant on each connected component of the moduli space $\mathscr{M}_{(H)}$. This in particular implies that the rational representation type $(H)$ already determines the complex representation type except possibly in the cases $1 \mathrm{~b}, 1 \mathrm{c}$, and $10 \mathrm{~b}$ of Table 4 . Indeed, in these cases this is not true, namely the two connected components correspond to two different complex representation types. More precisely the two types are in each of these cases given by representatives as follows (cf. the proofs of Proposition 2.19 and Lemma 2.5):

$$
\begin{aligned}
& \text { lb. }\left\langle\left(e_{2 m}, e_{2 m}^{-1}\right)\right\rangle,\left\langle\left(e_{2 m}, e_{2 m}\right)\right\rangle, \quad m=2,3 \\
& \text { lc. }\left\langle\left(e_{2 m}, e_{2 m}^{m-1}\right)\right\rangle,\left\langle\left(e_{2 m}, e_{2 m}^{m+1}\right)\right\rangle, \quad m=4,6 \\
& \text { 10b. }\left\langle(1, \pm 1),\left(\rho^{2}, \rho^{2}\right)\right\rangle, \quad\left\langle(1, \pm 1),\left(\rho^{2}, \rho^{-2}\right)\right\rangle \text { s }
\end{aligned}
$$

where in $1 \mathrm{~b}$ the type $\left\langle\left(e_{2 m}, e_{2 m}\right)\right\rangle$ belongs to the component $\{*\}$, and the pairs $(T, G)$ with $G \cong \mathbb{C}_{2 m}, m=2,3$, belonging to the component $\mathrm{GL}_{2}(\mathfrak{D}) \backslash \mathrm{GL}_{2}(\mathbb{C}) / \mathbb{C}^{* 2}$ are all special. In any case we see that the following holds.

Lemma 2.20. The moduli space of the pairs $(T, G)$ with fixed complex and rational representation types are connected if for the associated quadruple $F=(A, \mathfrak{D}, H, M)$ we have $\operatorname{rank}_{Q} A \leqq 4$. 


\section{§ 3. Special Pairs}

In this section we classify special pairs. Recall that a pair $(T, G)$ is said to be special if $f(G) \leqq \mathrm{SL}(E)$, where $f: G \rightarrow \mathrm{GL}(E)$ is the complex representation.

3.1. First we prove some general lemmas.

Lemma 3.1. The following conditions are equivalent. 1$)(T, G)$ is special, and 2) the minimal resolution $Z$ of the quotient normal analytic surface $T / G$ is a $K 3$ surface. Moreover if $a(T) \leqq 1$, then $(T, G)$ is necessarily special, where $a(T)$ is the algebraic dimension of $T$.

Proof. 1) is clearly equivalent to the following: 1)' $G$ leaves fixed a nonzero holomorphic 2-form $\alpha$ on $T$. Let $\pi: T \rightarrow T / G$ and $\gamma: Z \rightarrow T / G$ be the natural morphisms. If $Z$ is a $K 3$ surface and $\phi$ is a nowhere vanishing holomorphic 2 -form on $Z$, then $\left(\gamma^{-1} \pi\right)^{*} \psi$ gives a nonzero $G$-invariant holomorphic 2 -form on $T$. This shows that 2$) \rightarrow 1$ ). Conversely suppose that 1$)^{\prime}$ is true. Then $\alpha$ descends to a nonzero holomorphic 2 -form $\bar{\alpha}$ on $Z$ whose zeroes are contained in the inverse images $\gamma^{-1}\left(p_{i}\right)$ of the singular points $p_{i} \in T / G$. On the other hand, the stabilizer $G_{t}$ of each point $t \in T$ is naturally considered as a subgroup of $\mathrm{SL}(E)$ so that $T / G$ has only rational double points; thus $\bar{\alpha}$ cannot have zeroes contained in $\gamma^{-1}\left(p_{i}\right)$. Then $\bar{\alpha}$ is nowhere vanishing. On the other hand, since the eigenvalues of any element $(\neq e)$ of $G$ are not 1 on $E$, there is no $G$-invariant holomorphic 1 -form on $T$. This implies that $h^{1,0}(Z)=0$. It follows that $Z$ is a $K 3$ surface. For the last assertion see (the proof of) [14], Th. 3.1 a).

The first part of the next lemma is classical, being a special case of the so-called crystallographic restriction. The second part also holds in higher dimensions. Here we deduce it from Proposition 2. 14.

Lemma 3.2. Let $g$ be an automorphism of finite order $n$ of a two dimensional complex torus $T$. Then $\varphi(n) \leqq 4$; moreover if $g$ is special, $i_{0} e_{\text {. }}$, $f(g) \in \mathrm{SL}(E)$, then $\varphi(n) \leqq 2$, where $\varphi$ is the Euler function. 
Proof. Let $G$ be the abelian group generated by $g$ and -1 . Let $F$ be the quadruple associated to $(T, G)$. Then $F$ falls under 1 or 5 of Table 1. The first assertion then follows immediately. If $F$ is No. $1,4 \leqq m \leqq 6$, of Table 1 (which correspond to the case $G \cong \mathfrak{E}_{n}$ with $\varphi(n)=4)$, the corresponding complex representation type given in (15) shows that $(T, G)$ is not special. In other words, $\varphi(n) \leqq 2$ if $g$ is special.

Together with the well-known classification of finite subgroups of $\mathrm{SL}_{2}(\mathbb{C})$ this lemma gives us the following:

Lemma 3. 3. Let $(T, G)$ be a special pair. Then $G$ is isomorphic either to the cyclic group $\mathfrak{}_{k}$ of order $k$ with $k=2,3,4$ or 6 , or to one of the groups $\mathfrak{Q}, \mathfrak{I}$, or $\mathfrak{D}$.

Lemma 3.4. Let $(T, G)$ be any special pair with $-1 \in G$. Let $F=(A, \mathbb{D}, H, M)$ be a quadruple associated to $(T, G)$. Then if $G \cong \mathfrak{C}_{k}$, $k=3,4,6$ (resp. $\mathfrak{Q}, \mathfrak{I}$, or $\mathfrak{D}), F$ is isomorphic to one of the quadruples in Table 1, No. 1 with $m=2,3$ (resp. Nos. 12-14).

Proof. If $G \cong 夭_{k}$, then $A$ is clearly isomorphic to $\mathbb{Q}\left(e_{k}\right)$ and if $G \cong \mathscr{Q}, \mathfrak{I}$ or $\mathfrak{D}$, then $A$ is not commutative and cannot be isomorphic to $M_{2}(\$)$ since $G$ is never isomorphic to a subgroup of $\mathrm{GL}_{2}(\bigotimes)$. From these the lemma follows.

3. 2. Let $\mathbb{S}$ be any one of the groups $\mathfrak{E}_{k}, k=2,3,4,6, \mathfrak{Q}$, $\mathfrak{I}$, and D. Let $\mathscr{S}_{\mathbb{G}}$ be the set of isomorphism classes of special pairs $(T, G)$ such that $G \cong\left(S_{0}\right.$. We shall now determine the structure of $\mathscr{S}_{\mathbb{B}}$. We distinguish three cases according as $\mathbb{S}=$ i) $\mathfrak{夭}_{2}$, ii) $\mathfrak{夭}_{k}, k=3,4,6$ and iii) $\mathfrak{D}, \mathfrak{I}$, or $\mathfrak{D}$.

Case i). $\mathbb{S}=\mathfrak{C}_{2}$. In this case $T$ is arbitrary and $G=\langle-1\rangle . \quad \mathscr{I}_{\mathbb{\Xi}_{2}}$ is thus nothing but the moduli space $\mathrm{GL}_{4}(\mathbb{Z}) \backslash W \cong \mathrm{SL}_{4}(\mathbb{Z}) \backslash W^{+}$of the isomorphism classes of complex tori of dimension two. The action of $\mathrm{SL}_{4}(\mathbb{Z})$ on $W^{+}$is not properly discontinuous as is well-known.

Case ii). $\quad(S)=\mathfrak{C}_{k}, k=3,4,6$. Let $\mathfrak{D}=\mathfrak{b}_{k}$ be the maximal order of $\mathbb{Q}\left(e_{k}\right)$. With respect to the natural embedding $\mathfrak{D} \subseteq \mathbb{Q}\left(e_{k}\right) \cong \mathbb{C}, \Lambda:=\mathbb{D}^{2}$ is a lattice in $\mathbb{C}^{2}$ and is a free $\mathfrak{0}$-module. Thus the diagonal action 
of $e_{k} \in \mathfrak{o}^{\times}$on $\boldsymbol{C}^{2}$ preserves $\Lambda$ and descends to an automorphism $\hat{e}_{k}$ of the complex torus $\boldsymbol{C}^{2} / \Lambda$. Let $u$ be any point of the homogeneous complex manifold $U:=\mathrm{GL}_{2}(\boldsymbol{C}) /\left(\boldsymbol{C}^{* 2}\right)$ (cf. Proposition 2.17). Fix a representative $y$ of $u$ in $\mathrm{GL}_{2}(\boldsymbol{C})$. Then $y(i,-i) y^{-1}$ depends only on $u$; so we set $J(u)=y(i,-i) y^{-1} \in \mathrm{GL}_{2}(\boldsymbol{C})$ (cf. Notation). Then $J(u)^{2}=-1$, so that $J(u)$ defines a new complex structure on $\boldsymbol{C}^{2} \cong \boldsymbol{R}^{4}$ which is $\mathbf{0}^{-}$ linear, and hence, commutes with the action of $e_{k}$.

Let $T_{u}$ be the complex torus $\boldsymbol{C}^{2} / \Lambda$, but with the new complex structure induced by $J(u)$. Then $\hat{e}_{k}$ is holomorphic also on $T_{u}$. Therefore we have the natural embedding $\left\langle\hat{e}_{k}\right\rangle \longrightarrow$ Aut $T_{u}$. Let $G_{u}$ be the image of this embedding. Then the resulting pair $\left(T_{u}, G_{u}\right)$ is special with $G_{u} \cong \mathfrak{夭}_{k}$ (cf. (15)). Thus we have a family $\left\{\left(T_{u}, G_{u}\right)\right\}_{u \in U}$ of special pairs parametrized by $U$. By construction this is just the holomorphic family (3) associated to the quadruple $\left(\boldsymbol{Q}\left(e_{2 m}\right), \tilde{\mathfrak{D}}, \mathfrak{D}, \mathfrak{D}^{2}\right)$ restricted to the component $U$ (cf. Proposition 2.17 and (15)). Therefore we have almost obtained the following:

Proposition 3.5. Let $(T, G)$ be a special pair with $G \cong \mathfrak{C}_{k}, k=3,4$, or 6. Then there exists a point $u \in U$ such that $(T, G) \cong\left(T_{u}, G_{u}\right)$. Moreover, for any $u, u^{\prime} \in U,\left(T_{u}, G_{u}\right)$ and $\left(T_{u^{\prime}}, G_{u^{\prime}}\right)$ are isomorphic if and only if there exist representatives $y, y^{\prime}$ in $\mathrm{GL}_{2}(\boldsymbol{C})$ of $u, u^{\prime}$ respectively and an element $\gamma \in \mathrm{GL}_{2}(\mathrm{D})$ such that $y=\gamma y^{\prime}$ or $y=\gamma \bar{y}^{\prime}$, where $\bar{y}^{\prime}$ denotes the complex conjugate matrix of $y^{\prime}$.

Remark 3.6. In particular the moduli space $\mathscr{S}_{\mathbb{\Xi}_{k}}$ is naturally identified with the locally homogeneous space

$$
\left(\mathrm{GL}_{2}(\mathfrak{D}) \backslash \mathrm{GL}_{2}(\boldsymbol{C}) /\left(\boldsymbol{C}^{*}\right)^{2}\right) / \mathbb{E}_{2},
$$

where the action of $\mathfrak{C}_{2}$ on $\mathrm{GL}_{2}(\mathfrak{D}) \backslash \mathrm{GL}_{2}(\boldsymbol{C}) /\left(\boldsymbol{C}^{*}\right)^{2}$ is induced by the complex conjugation on $\mathrm{GL}_{2}(\boldsymbol{C})$. Since the action of $\mathrm{GL}_{2}(\mathfrak{d})$ on $\mathrm{GL}_{2}(\boldsymbol{C}) /\left(\boldsymbol{C}^{*}\right)^{2}$ is not properly discontinuous $\mathscr{S}_{\tilde{\varepsilon}_{k}}$ has no structure of analytic spaces.

Proof. Let $(T, G)$ be a pair from $\mathscr{S}_{\mathbb{\varepsilon}_{3}}$. Then $\left(T, G^{\prime}\right)$ with $G^{\prime}=\langle G,-1\rangle$ is in $\mathscr{S}_{\mathbb{\Xi}_{6}}$ and the correspondence $(T, G) \rightarrow\left(T, G^{\prime}\right)$ gives a bijection of $\mathscr{S}_{\mathbb{E}_{3}}$ and $\mathscr{S}_{\mathbb{G}_{6}}$. Therefore, we may only consider the cases $k=4$ or 6 . Since $(T, G)$ is special, the result then follows from Lemma 3. 4, Lemma 2.3, Proposition 2. 19 and (15). 
In the above description of the pairs in $\mathscr{S}_{\mathbb{E}_{k}}$ the structure of $T$ as a complex manifold is not explicit enough. As a complementary result we shall give here a brief description of the elements of $\mathscr{S}_{\mathbb{x}_{k}}$ in terms of their period matrices according to [6]. Let $\Omega$ be a $2 \times 4$ complex matrix with $\operatorname{det}\left(\frac{\Omega}{\Omega}\right) \neq 0$. Then the column vectors of $\Omega$ generate a lattice $\Lambda$ of $\mathbb{C}^{2}$. Let $T=\mathbb{C}^{2} / \Lambda$ be the resulting complex torus with period $\Omega$. Then any element $g_{0} \in \mathrm{GL}_{2}(\mathbb{C})$ preserving $\Lambda$ defines an automorphism $g$ of $T$. We now consider the following two cases.

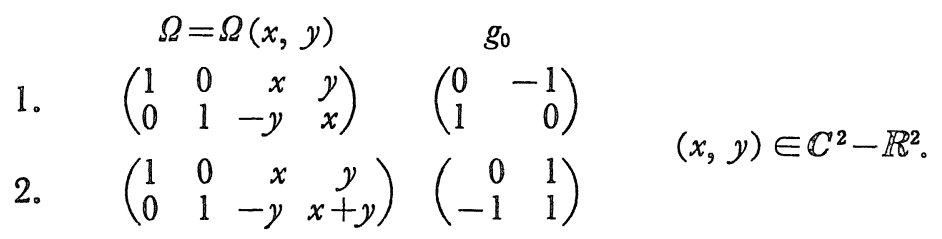

Here $g_{0}$ is of order 4 and 6 in the respective cases with $\operatorname{det} g_{0}=1$. Hence if we set $T=T(\Omega(x, y))$ and $G=\left\langle g_{0}\right\rangle$, the resulting pair $(T, G)_{(x, y)}$ is a special pair in $\mathscr{S}_{\mathbb{\varepsilon}_{k}}, k=4,6$. Conversely the following is easily verified (cf. [6], p. 220).

Proposition 3.7. Any special pair $(T, G)$ with $G \cong \mathfrak{C}_{k}, k=4,6$, is isomorphic to $(T, G)_{(x, y)}$ for some $(x, y) \in \mathbb{C}^{2}-\mathbb{R}^{2}$ as above.

For instance by setting $y=0$ in 1 and 2 we see that for any elliptic curve $E$ the product $E \times E$ always admits a special action of $\mathfrak{夭}_{k}, k=4,6$ (cf. Table 8). On the other hand, the automorphism $\left(e_{k}, e_{k}^{-1}\right)$ defines a special action of $\mathfrak{S}_{k}$ on $E_{e_{k}} \times E_{e_{k}}$. More generally the following is true:

Lemma 3.8. Let $T$ be a complex torus of dimension 2 which is obtained as an extension of $E_{e_{k}}$ by $E_{e_{k}}, k=4,6$. Then $T$ admits a special action of $\mathfrak{\Im}_{k}$ 。

Proof. Set $\lambda=e_{k}$. Choosing a suitable basis of $H_{1}(T, \mathbb{Z})$ we can find a period matrix $\Omega$ of $T$ in the form

$$
\Omega=\left(\begin{array}{llll}
1 & \lambda & u & v \\
0 & 0 & 1 & \lambda
\end{array}\right), \quad u, v \in \mathbb{C} \text { 。 }
$$


Further, if $u \neq 0$, then after multiplying $\Omega$ by $\left(\begin{array}{cc}1 & -1 / u \\ 0 & 1\end{array}\right) \in \mathrm{GL}_{2}(\boldsymbol{C})$ from the left we may always assume that $u=0$. Then set $g=\left(\begin{array}{ll}\lambda & -v \\ 0 & \lambda^{-1}\end{array}\right) \in$ $\mathrm{SL}_{2}(\mathcal{C})$. We see readily that $g$ is of order $k$ and $g \Omega=\Omega \nu$ for some $\nu \in \mathrm{GL}_{4}(\mathbb{Z})$. This implies that $g$ defines a special pair $(T, G)$ with $G \cong \bigodot_{k}$ as desired.

Remark 3.9. The lemma can be proved more intrinsically, using the fact that $G$ acts on the image of the natural homomorphism $H^{1}\left(C^{\prime}, \mathcal{O}_{C^{\prime}}\right) \rightarrow H^{1}\left(C^{\prime}, \mathcal{O}_{C^{\prime}}(C)\right)$, which parametrizes the universal family of the extensions of $C$ by $C^{\prime}$ as a complex Lie group.

The general extension of $E_{e_{k}}$ by $E_{e_{k}}$ is of algebraic dimension 1. On the other hand, any complex torus $T$ of algebraic dimension 1 contains a unique subtorus $C^{\prime}$ (up to translations), and the quotient homomorphism $f: T \rightarrow C:=T / C^{\prime}$ gives the algebraic reduction of $T$. Thus by the above lemma if $C \cong C^{\prime} \cong E_{e_{k}}, T$ admits a special action of $\mathfrak{\wp}_{k}$. More precisely we have the following:

Proposition 3.10. Let $T$ be a complex torus of dimension two with algebraic dimension $a(T)=1$. Let $f: T \rightarrow C$ be the algebraic reduction of $T$ with kernel $G^{\prime}$ as above. Then for $k=4,6$, Aut $T \cong \mathfrak{\Im}_{k}$ if and only if $C \cong C^{\prime} \cong E_{e_{k}}$. Otherwise Aut $T \cong\langle-1\rangle \cong \mho_{2}$.

Proof. Since $f$ is intrinsic for $T$, there exists natural homomorphisms $u:$ Aut $T \rightarrow$ Aut $C$ and $u^{\prime}:$ Aut $T \rightarrow$ Aut $C^{\prime}$. We show that these are both injective. Indeed, if there exists an element $g$ in the kernel of $u$ (resp. $u^{\prime}$ ) other than the identity, the kernel (resp. image) of the endomorphism $g-1$ turns out to be a subtorus of dimension 1 which is transversal to the fibers of $f$, which is a contradiction since $a(T)=1$. From this the necessity of the condition and the last assertion already follow. The sufficiency follows from Lemma 3.8.

3. 3. Case iii)。 $(S=\mathfrak{N}, \mathfrak{I}$, or $\mathfrak{D}$. Recall the natural identifications

$$
X=\mathbb{H}^{\times} / \mathbb{C}^{*}=\mathbb{P}^{1},
$$

where $X=\left\{q \in \mathbb{H} ; q^{2}=-1\right\}$ (cf. (12)). Let $q$ be any point of $X$. Then the right multiplication by $q$ defines a complex structure $J(q)$ 
on $H \cong \mathbb{R}^{4}$ 。 Set

$$
\Lambda_{\mathrm{D}}=\mathfrak{a}_{0}, \Lambda_{\mathfrak{x}}=\mathfrak{a}_{,}, \Lambda_{\triangleright}=\mathfrak{b}
$$

(cf. (4)). Consider $\Lambda_{\circledast}$ as a lattice in $\mathbb{H}_{1} \cong \mathbb{R}^{4} . J(q)$ then defines the structure of a complex torus $T_{q}=T_{\mathbb{G}, q}$ on the real torus $\mathbb{T}:=\mathbb{H} / \Lambda_{\mathbb{B}}$ 。 Let $\Lambda_{\mathbb{B}}^{\times}$be the unit group of $\Lambda_{\mathbb{G}}$. Then $\Lambda_{\mathbb{G}}^{\times} \cong \mathbb{S}$ by Lemma 2.6. The left multiplication by elements of $\Lambda_{\Theta}^{\times}$on $\mathbb{H}$ preserve $\Lambda_{\mathfrak{G}}$ and obviously commutes with $J(q)$; thus it induces canonically an automorphism of $T_{q}$, so that we have the natural embedding $\Lambda_{\mathbb{G}}^{\times} \longrightarrow$ Aut $T_{q}$. Let $G_{q}=G_{\mathbb{G}, q}$ be the image of $\Lambda_{\circledast}^{\times}$. Then

$$
U_{G}=\left\{\left(T_{q}, G_{q}\right)\right\}_{q \in X}
$$

is a family of special pairs in $\mathscr{S}_{\text {}}$ parametrized by $X \cong \mathbb{P}^{1}$. Indeed, by construction this is nothing but the holomorphic family (3) parametrized by $X \cong W_{F}$ associated to the quadruple $F$, where $F=\left(A, \Lambda_{\mathbb{G}}, \Lambda_{\Theta}^{\times}\right.$, $\left.\Lambda_{\mathfrak{G}}\right)$ with $A=\mathbb{Q}[1, i, j, k]$ if $\mathbb{S}=\mathfrak{Q}$ or $\mathfrak{T}$ and $=\mathbb{Q}[1, i, h, l]$ if $\mathbb{S}=\mathfrak{D}$. We write $X=X_{\otimes}$ also. Now define the subgroups $\Gamma_{1}$ and $\Gamma_{2}$ of $\mathbb{H}^{\times} \times$by

$$
\begin{aligned}
& \Gamma_{1}=\left\langle\mathfrak{a}^{\times},(1+i) / \sqrt{2}\right\rangle \cong \mathfrak{D} \\
& \Gamma_{2}=\left\langle\mathfrak{b}^{\times},(\sqrt{3}+k) / 2\right\rangle \cong \tilde{\mathfrak{D}}
\end{aligned}
$$

(cf. (5)). Set $\Gamma_{\circledast}=\Gamma_{1}$ if $\mathbb{S}=\mathfrak{Q}$ or $\mathfrak{T}$ and $=\Gamma_{2}$ if $\mathbb{S}=\mathfrak{D}$. Then the induced action of $\Gamma_{\Theta}$ on $X$ by inner automorphisms factors through the quotient $\vec{\Gamma}_{\Theta}:=\Gamma_{\Theta} /\{ \pm 1\}$

Now we call a special pair $(T, G)$ maximal if $G$ is not contained in another finite subgroup $G^{\prime}$ of Aut $T$ such that $\left(T, G^{\prime}\right)$ is again special. Note that when $G$ is not cyclic, $(T, G)$ is not maximal only in the case where $G^{\prime} \cong \mathfrak{I}$ and $G$ is the unique normal subgroup of $G^{\prime}$ isomorphic to $\mathscr{Q}$ (in the above notation). Then combining Lemma 2.3, Lemma 3.4 and Proposition 2.19 we get the following:

Theorem 3.11. Let $(T, G)$ be a special pair with $G \cong \circlearrowleft \&$. If $(T, G)$ is maximal, then $(T, G)$ is isomorphic to $\left(T_{q}, G_{q}\right)$ for some point $q \in X_{\text {ब. }}$. Moreover for $q, q^{\prime} \in X\left(T_{q}, G_{q}\right)$ and $\left(T_{q^{\prime}}, G_{q^{\prime}}\right)$ are isomorphic to each other if and only if $q^{\prime}=\gamma(q):=\gamma q \gamma^{-1}$ for some $\gamma \in \Gamma_{\Theta}$. If $(T, G)$ is not maximal, then there exists a point $q$ of $X_{\mathfrak{\Sigma}}$ such that $(T, G)$ is isomorphic to $\left(T_{q}, G_{q}^{0}\right)$, where $T_{q}=T_{x, q}$ and $G_{q}^{0}$ is the unique normal subgroup of $G_{q}=G_{\mathfrak{x}, q}$ isomorphic to $\mathfrak{Q}$. Further $\left(T_{q}, G_{q}^{0}\right)$ and $\left(T_{q^{\prime}}, G_{q^{\prime}}^{0}\right)$ are isomorphic to each other if and only if so are $\left(T_{q}, G_{q}\right)$ and $\left(T_{q^{\prime}}, G_{q^{\prime}}\right)$. 
Remark 3.12. In particular 1) $\mathscr{S}_{\mathrm{D}}$ is naturally identified with the disjoint union $\bar{\Gamma}_{\mathrm{o}} \backslash X \Perp \bar{\Gamma}_{\mathrm{z}} \backslash X \cong \mathbb{P}^{1} \Perp \mathbb{P}^{1}$ and 2) for $\mathbb{G}=\mathfrak{I}$ or $\mathscr{D}, \mathscr{S}_{\mathbb{G}}$ is naturally identified with $\bar{\Gamma}_{\mathbb{B}} \backslash X \cong \mathbb{P}^{1}$. (Recall that $\bar{\Gamma}_{\mathrm{Q}}=\bar{\Gamma}_{\mathrm{x}} \cong \mathbb{S}_{4}$ and $\bar{\Gamma}_{\triangleright} \cong \mathscr{D}_{6}$.)

Let $b_{\circledast}: X \rightarrow \bar{\Gamma}_{\circledast} \backslash X$ be the covering map. Since $\bar{\Gamma}_{\circledast} \cong \mathbb{S}_{4}$ or $\mathscr{D}_{6}, b_{\circledast}$ must have exactly three branch points, say $p_{1}, p_{2}, p_{3}$, on $\bar{\Gamma}_{\mathbb{s}} \backslash X$. In the next proposition we shall identify these points $p_{\alpha}=p_{\theta, \alpha}$ by specifying a point $q_{\alpha}=q_{\Theta, \alpha}$ of $b_{\Theta}^{-1}\left(p_{\alpha}\right)$ for each $\alpha$. Moreover we shall also determine the stabilizer $\bar{\Gamma}_{\alpha}=\bar{\Gamma}_{\Theta, \alpha}$ in $\bar{\Gamma}_{\theta}$ at $q_{\alpha}$ and the isomorphism class of the corresponding complex torus $T_{\alpha}:=T_{q_{\alpha}}$.

Proposition 3.13. In the above notation $q_{\alpha}, \bar{\Gamma}_{\alpha}$, and $T_{\alpha}$ are given as in the following table.

Table 5

\begin{tabular}{|c|c|c|c|c|}
\hline (S) & & $\alpha=1$ & $\alpha=2$ & $\alpha=3$ \\
\hline $\mathfrak{Q}$ or $\mathfrak{T}$ & $\begin{array}{l}q_{\alpha} \\
\bar{\Gamma}_{\alpha}\end{array}$ & $\begin{array}{c}i \\
\langle(1+i) / \sqrt{2}\rangle^{-} \cong \Im_{4}\end{array}$ & $\begin{array}{l}(i+j) / \sqrt{2} \\
\langle(i+j) / \sqrt{2}\rangle^{-} \cong \mathfrak{S}_{2}\end{array}$ & $\begin{array}{l}(i+j+k) / \sqrt{3} \\
\langle t\rangle^{-\cong} \mathfrak{\mho}_{3}\end{array}$ \\
\hline $\begin{array}{l}\mathfrak{Q} \\
\mathfrak{I}\end{array}$ & $T_{\alpha}$ & $\begin{array}{l}E_{i} \times E_{i} \\
E_{i} \times E_{i}\end{array}$ & $\begin{array}{l}E_{\sqrt{\sqrt{2} i}} \times E_{\sqrt{2} i} \\
E_{\sqrt{\sqrt{2} i} i} \times E_{\sqrt{2} i}\end{array}$ & $\begin{array}{l}E_{\rho} \times E_{\sqrt{3} i} \\
E_{\rho} \times E_{\rho}\end{array}$ \\
\hline () & $\begin{array}{l}q_{\alpha} \\
\bar{\Gamma}_{\alpha} \\
T_{\alpha}\end{array}$ & $\begin{array}{l}\quad \quad i \\
\langle i\rangle^{-} \cong \mathfrak{E}_{2} \\
E_{i} \times E_{i}\end{array}$ & $\begin{array}{c}j \\
\langle j\rangle^{-} \cong \Im_{2} \\
E_{\sqrt{3} i} \times E_{\sqrt{3} i}\end{array}$ & $\begin{array}{l}\quad k \\
\langle(\sqrt{3}+k) / 2\rangle^{-} \cong \mathfrak{E}_{6} \\
E_{\rho} \times E_{\rho}\end{array}$ \\
\hline
\end{tabular}

Here \langle\rangle$^{-}$denotes the quotient group \langle\rangle$/\{ \pm 1\}$.

Proof. It is immediate to see that if we define $q_{\alpha}$ as in the table, then the stabilizer $\bar{\Gamma}_{\alpha}$ at $q_{\alpha}$ is just as in the table, and $q_{\alpha}$ are not mutually equivalent under the action of $\bar{\Gamma}_{\mathbb{G}}$. Hence we may take $p_{\alpha}=b\left(q_{\alpha}\right)$. Let $J:=J_{\alpha}$ be the right multiplication by $q_{\alpha}$. Then for each $\alpha$ we can take the following set of 4 elements as a $\mathbb{Z}$-basis of $\Lambda_{\mathbb{G}}, \mathbb{S}=\mathfrak{Q}, \mathfrak{T}, \mathfrak{D}$.

1) $\mathbb{S}=\mathfrak{Q}: \alpha=1\{1, J(1), j, J(j)\}, \quad \alpha=2\{1-i, \sqrt{2} J(1), j, \sqrt{2} J(j)\}$,

$$
\alpha=3\{1-i,(\rho J)(1-i), j, \sqrt{3} J(1-i)\} .
$$


2) $\mathbb{S}=\mathfrak{T}: \quad \alpha=1 \quad\{1, J(1), t, J(t)\}, \quad \alpha=2\{1, \sqrt{2} J(1), t, \sqrt{2} J(t)\}$, $\alpha=3\{1, \rho(J)(1), j, \rho(J) j\}$ 。

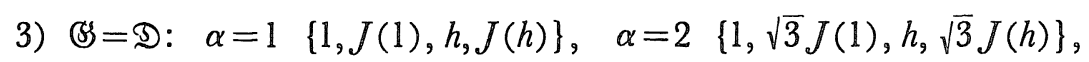
$\alpha=3\{1, \rho(J)(1), h, \rho(J)(h)\}$,

where $\rho(J)=(1+\sqrt{3} J) / 2$. From this we can identify the structure of $T_{\alpha}$ as in the table readily.

Remark 3.14. Using the above mentioned basis of $\Lambda_{\mathbb{B}}$ it is also straightforward to obtain an explicit description of $G_{\alpha}$ itself (cf. Table 9 below). Let $(T, G)$ be any maximal special pair with $G$ noncyclic。 If $G$ is properly contained in its normalizer in Aut $T,(T, G)$ is isomorphic to one of $\left(T_{\alpha}, G_{\alpha}\right)$ above, as follows from the definition of $\left(T_{\alpha}, G_{\alpha}\right)$ and the remark at the end of 2.1 .

As the above remark shows the complex tori $T_{\alpha}$ in Table 5 , or more precisely the associated pairs $\left(T_{\alpha}, G_{\alpha}\right)$, play a distinguished role in the study of automorphism groups of complex tori of dimension 2 in general. The next proposition provides us such an example (cf. also Table 9 and Example 7.9).

Proposition 3.15. Let $(T, G)$ be a maximal special pair with $G \cong \mathfrak{D}_{\text {, }}$ $\mathfrak{I}$, or $\mathfrak{D}$. Then $T$ admits a $G$-invariant principal polarization if and only if $(T, G)$ is isomorphic to $\left(T_{1}, G_{1}\right)$ in case $G \cong \mathfrak{Q}$, to $\left(T_{2}, G_{2}\right)$ in case $G \cong \mathfrak{I}$, and either to $\left(T_{2}, G_{2}\right)$ or $\left(T_{3}, G_{3}\right)$ in case $G \cong \mathfrak{D}$. In this case such a polarization is unique up to constant multiples and is invariant also under the normalizer of $G$ in Aut $T$.

The proof will be given at the end of Section 6 .

3. 4. We shall now generalize the preceding classification also to pairs $(T, G)$, where $G$ is a finite subgroup of the group $A(T)$ of affine transformations of $T$ (instead of Aut $T$ )。A(T) is naturally the semi-direct product $A(T)=A_{0}(T) \rtimes$ Aut $T$, where $A_{0}(T)$ is the group of translations of $T$. Let $w: A(T) \rightarrow$ Aut $T$ be the natural projection. Then any pair $(T, G)$ with $G$ a finite subgroup of $A(T)$ gives rise to the pair $(T, w(G))$ in the original sense, and it is called 
special if $(T, w(G))$ is. In what follows we always assume that $G$ contains no translations, i. e., $G \cap A_{0}(T)=\{e\} . G$ is then isomorphic to $w(G)$. We say that two such (generalized) pairs $(T, G)$ and $\left(T^{\prime}, G^{\prime}\right)$ are isomorphic if there exists an affine isomorphism $v: T \rightarrow T^{\prime}$ such that $v G v^{-1}=G^{\prime}$. Then the following is obvious.

Lemma 3.16. 1) A (generalized) pair $(T, G)$ is isomorphic to a pair $\left(T^{\prime}, G^{\prime}\right)$ with $G^{\prime} \cong$ Aut $T^{\prime}$ if and only if $G$ has a fixed point, and 2) the pairs $(T, G)$ and $\left(T^{\prime}, G^{\prime}\right)$ with $G \subseteq$ Aut $T$ and $G^{\prime} \cong$ Aut $T^{\prime}$ are isomorpic as generalized pairs if and only if they are isomorphic in the original sense.

In the following we shall call a generalized pair simply a pair. By virtue of the above lemma and Theorem 3.11 we have only to consider the case where $G$ has no fixed points. First we shall give examples, which eventually turn out to exhaust all the possible cases. We consider the universal family $U_{\mathfrak{x}}=\left\{\left(T_{q}, G_{q}\right)\right\}_{q \in x_{\mathfrak{x}}}$ mentioned earlier (cf. (17)). Let $\mathbb{T}=\boldsymbol{H} / \Lambda$ be the underlying real torus, where $\Lambda=\Lambda_{\boldsymbol{x}}$. For any $\lambda \in \mathrm{Aut}_{\boldsymbol{Z}} \Lambda$ and $r \in \mathbb{H}$ the real affine automorphism $x \rightarrow \lambda x+r$ of $\boldsymbol{H}$ descends to an affine automorphism of the real torus $\mathbb{T}$, which we shall denote by $(\lambda ; r)$, where $r$ is identified with the residue class modulo $\Lambda$. For simplicity we write $\lambda$ for $(\lambda ; 0)$.

We denote by $A(\mathbb{T})$ and $A_{0}(\mathbb{T})$ the group of real affine automorphisms, and the group of translations, of $\mathbb{T}$ respectively. Then $A(\mathbb{T})$ is naturally the semidirect product $A\left(\mathbb{T}^{\prime}\right)=\mathrm{Aut}_{z} \Lambda \ltimes A_{0}\left(\mathbb{T}^{\prime}\right)$. For any $q \in X=X_{\approx}$ if $\lambda \in \Lambda^{\times} \cong \mathbb{I}$ (left multiplication), $(\lambda ; r)$ clearly is biholomorphic on $T_{q}$ so that we have the natural embedding $j_{q}: \Lambda^{\times} \ltimes A_{0}(\mathbb{T}) \longrightarrow$ $A\left(T_{q}\right)$. Now define the subgroups $Q_{0}$ and $T_{0}$ of $\Lambda^{\times} \ltimes A_{0}(T)$ by

$$
\begin{array}{lc}
Q_{0}=\{ \pm 1, \pm i,( \pm j ; a), & ( \pm k ; a)\}, \quad a=(1+i) / 2 \\
T_{0}=\left\langle Q_{0},(t ; s / 2)\right\rangle, & s=(1+i-j+k) / 2 .
\end{array}
$$

Then $Q_{0} \cong \mathscr{Q}, T_{0} \cong \mathfrak{I}$ and the inclusion $Q_{0} \cong T_{0}$ is isomorphic to the natural inclusion $\mathfrak{Q} \subseteq \mathfrak{T}$. Moreover for any $q, Q_{0}$ and $T_{0}$ define via $j_{q}$ finite subgroups $\hat{H}_{q}$ and $\hat{G}_{q}$ of $A\left(T_{q}\right)$ respectively. Let $\Gamma_{0}$ be the subgroup of $\Gamma_{x}$ defined by

$$
\Gamma_{0}=\left\langle\Lambda_{0}^{\times},(1+i) / \sqrt{2}\right\rangle
$$

(cf. Table 2). Then we have $\bar{\Gamma}_{0}:=\Gamma_{0} /\{ \pm 1\} \cong \mathfrak{D}_{4} . \hat{H}_{q}$, and hence $\hat{G}_{q}$ also, have no fixed point on $T_{q}$. Conversely, we have the following: 
Theorem 3.17. Let $(T, G)$ be a special pair with $G \cong A(T)$. Suppose that $G$ has no fixed points. Then $G$ is isomorphic to $\mathfrak{Q}$ or $\mathfrak{I}$, and if $G \cong \mathfrak{Q}$ (resp. I),$(T, G)$ is isomorphic to $\left(T_{q}, \hat{H}_{q}\right)$ (resp. $\left(T_{q}, \hat{G}_{q}\right)$ ) for some $q \in X$. Moreover $\left(T_{q}, \hat{H}_{q}\right)$ and $\left(T_{q}, \hat{H}_{q^{\prime}}\right)$ (resp. $\left(T_{q}^{\prime}, \hat{G}_{q}\right)$ and $\left.\left(T_{q^{\prime}}, \hat{G}_{q^{\prime}}\right)\right)$ are isomorphic if and only if $q=h q^{\prime} h^{-1}$ for some $h \in \Gamma_{0}$. In particular the moduli spaces are given by $\bar{\Gamma}_{0} \backslash X \cong \mathbb{P}^{1}$ in both cases.

Remark 3.18. In [5;VII] Enriques-Severi has classified those special pairs $(T, G)$ for which $T$ is the jacobian of a complete nonsingular curve of genus 2 and $G$ preserves the associated principal polarization. Especially they showed that if $G$ is isomorphic to $\mathscr{Q}$ or $\mathfrak{I}$ (resp. D), then there exist 3 (resp. a unique) isomorphism classes, which is in accordance with our Theorems 3.11 and 3.17. In fact, these pairs turn out to be given in the above notations as follows (cf. Proposition 3.15):

1) $G \cong \mathbb{T}:\left(T_{2}, G_{2}\right),\left(T_{2}, \hat{G}_{2}\right):=\left(T_{q_{2}}, \hat{G}_{q_{2}}\right)$ and $\left(T_{q_{2}^{\prime}}, \hat{G}_{q_{2}^{\prime}}\right)$, where $q_{2}=(i+j) / \sqrt{2}$ and $q_{2}^{\prime}=(j+k) / \sqrt{2}$.

2) $G \cong \mathscr{Q}$ : the pairs obtained from the above triples by restriction to the unique normal subgroups isomorphic to $\mathscr{Q}$; in this case $(T, G)$ is not maximal.

3) $G \cong \mathfrak{D}:\left(T_{2}, G_{2}\right)$.

3. 5. The rest of this section is devoted to the proof of Theorem 3. 17. We need several lemmas. Let $(T, G)$ be any pair. For any subgroup $G$ of $A(T)$ we shall denote by Fix $G$ the fixed point set of $G$. When $G=\langle\alpha\rangle$, we write Fix $\alpha$ instead.

Let $(T, G)$ be any special pair. By the proof of Lemma 3.1 and Lemma 3.3 T/G has only rational double points of type $A_{k}, D_{4}, D_{5}$ or $E_{6}$; further if $r: Z \rightarrow T / G$ is the minimal resolution, $Z$ is a $K 3$ surface. Let $n_{k}$ be the number of singular points of $T / G$ of type $A_{k-1}$, $k \geqq 2$, and $n_{\circledast}$ the number of singular points of type $D_{4}, D_{5}$ or $E_{6}$ according as $\mathbb{S}=\mathfrak{Q}, \mathfrak{D}$, or $\mathfrak{I}$. Then we have the following relation

$$
\chi(Z)=(\chi(T)-\chi(\operatorname{Sing} G)) /|G|+\chi\left(r^{-1}(\pi(\operatorname{Sing} G)),{ }^{*}\right)
$$

where $\chi(X),|G|$ and $\pi$ denote respectively the topological Euler characterestic of $X$, the order of $G$, and the natural projection $T \rightarrow T / G$.

*) Sing $G$ denotes the set of points whose stabilizer group is nontrivial. 
From this, as in Nikulin $[14, \S 5]$, we get easily the following formula:

$$
24=-\left(\sum_{k \geqq 2} n_{k} / k+\sum_{\Theta} n_{\Theta} /|G|\right)+\sum_{k \geqq 2} k n_{k}+5 n_{\circlearrowleft}+6 n_{刃}+7 n_{\mathfrak{\tau}} .
$$

Lemma 3. 19. Let $(T, G)$ be any special pair. Let $\mathbb{S}=\mathfrak{Q}$, $\mathfrak{T}$, or $\mathfrak{D}$. Let $f_{k}$ and $f_{\circledast}$ be the numbers of points of $x$ of $T$, whose stabilizer $G_{x}$ at $x$ is isomorphic to $\mathfrak{S}_{k}$ and \&S respectively. Then the possible combinations of the nonzero $f_{k}$ and $f_{\circledast}$ are as follows; 1) $\left.G \cong \mathfrak{F}_{2}: f_{2}=16,2\right) G \cong \mathfrak{\mho}_{3}$ : $f_{3}=9$, 3) $G \cong \mathfrak{\Im}_{4}: f_{2}=12, f_{4}=4$, 4) $G \cong \mathfrak{\Im}_{6}: f_{2}=15, f_{3}=8, f_{4}=1, f_{6}=1$,

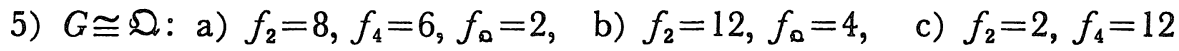
6) $G \cong \mathfrak{T}$ : a) $f_{2}=12, f_{3}=32, f_{\mathrm{o}}=3, f_{\mathfrak{x}}=1$, b) $f_{3}=32, f_{4}=12, f_{6}=4$, 7) $G \cong \mathfrak{I}: f_{2}=6, f_{3}=8, f_{4}=9$.

Proof. It is easy to compute all the possible values of $n_{k}$ and $n_{\text {o }}$ satisfying (19). Then using the relations $|G| n_{k}=k f_{k}$ and $|G| n_{\circledast}=|G| f_{\circledast}$, we obtain the lemma easily.

We note that when $G \cong \Im_{2}$, Fix $G$ is just the 2-torsion groups of $T$ 。 From this lemma we deduce easily the following:

Lemma 3.20. Let $(T, G)$ be a special pair. 1) If $G \cong \mathbb{F}_{4}$, Fix $G$ is contained in the 2-torsion group of $T$ and \#(Fix $G)=4.2)$ If $G \cong \mathfrak{Q}$ and Fix $G \neq \emptyset, \#($ Fix $G)=2$ or 4 according as $(T, G)$ is maximal or not. 3) If $G \cong \mathfrak{I}$ and Fix $G=\emptyset$, then Fix $G_{1}=\emptyset$ for the unique normal subgroup $G_{1}$ of $G$ isomorphic to $\mathfrak{Q}$. 4) If Fix $G=\emptyset$, then $G \cong \mathfrak{Q}$ or $\mathfrak{I}$.

Using this lemma we shall show the following:

Lemma 3.21. Let $(T, G)$ be a special pair with $G \cong \mathfrak{D}$. Let $G^{\prime}:=$ $w(G) \subseteq$ Aut $T$. Let $\alpha, \beta$ be generators of $G^{\prime}$ such that $G \cap G^{\prime}=\langle\alpha\rangle$ in $A(T)$. Then $G$ is generated by $\alpha$ and $(\beta ; r)$ for some $r \in$ Fix $G^{\prime}$. Moreover if Fix $G=\emptyset,\left(T, G^{\prime}\right)$ is not maximal.

Proof. It is clear that $G=\langle\alpha,(\beta ; r)\rangle$ for some $r \in T$. Let $\beta_{0}=$ $(\beta ; r)$. Then $\beta_{0}$ should satisfy $\beta_{0}^{2}=-1$ and $\alpha \beta_{0} \alpha^{-1}=\beta_{0}^{-1}$; these conditions are then equivalent respectively to: $\beta r=-r$ and $\beta \alpha r=r$. From the latter and Lemma 3.20 it follows that $r$ is a 2-torsion point of $T$. Hence $r=-r$ and $r \in$ Fix $G^{\prime}$. This proves the first assertion.

Suppose now that $\left(T, G^{\prime}\right)$ is maximal. Then by Lemma 3.20 
there exists a point $x \in$ Fix $\alpha$ with $x \notin$ Fix $G^{\prime}$. Then we have Fix $\alpha$ $=\{0, r, x, x+r\}$ (cf. Lemma 3.20). On the other hand, since $\beta$ preserves Fix $\alpha$ and $\beta x \neq 0, r, x$ we must have $\beta(x)=x+r$, i. e., $\beta_{0}(x)=x$, namely $x \in$ Fix $G$. Thus Fix $G \neq \emptyset$.

Lemma 3.22. Let $(T, G)$ be any special pair with $G \cong \mathfrak{Q}$ and Fix $G=\emptyset$. Let $T$ be the underlying real torus. Then $G$ is conjugate to $Q_{0}$ in $A(\mathbb{T})$ with respect to the representation $\mathbb{T}=\mathbb{H} / \Lambda_{\mathfrak{\Sigma}}$ of $\mathbb{T}$.

Proof. Let $G^{\prime}=w(G)$. Then by passing to another pair which is isomorphic to $(T, G)$ if necessary (cf. Lemmas 3.16 and 3.20) we may assume that $G \cap G^{\prime}=\langle\alpha\rangle$ in $A(T)$ for some element $\alpha$ of order 4 . Then by Lemma 3.21 we may write $G=\langle\alpha,(\beta ; r)\rangle$ for some element $\beta \in G^{\prime}$ and $r \in$ Fix $G^{\prime}$ with $r \neq 0$; by Lemma 3.21 it further follows that $\left(T, G^{\prime}\right)$ is not maximal. Hence by Theorem 3.11 we may assume that the underlying real action on $\mathbb{T}=\mathbb{H} / \Lambda_{\mathfrak{x}}$ is induced by the left multiplications by elements of $\Lambda_{0}^{\times} \subseteq \Lambda_{\approx}^{\times}$. We may therefore assume that $G^{\prime}=\Lambda_{0}^{\times}$and hence that $G$ is a subgroup of $\Lambda_{0}^{\times} \ltimes A_{0}(\mathbb{T})$. Then $r$ is one of $(1+\omega) / 2, \omega=i, j$, or $k$.

On the other hand, the automorphism group Aut $\Lambda_{0}^{\times}$of $\Lambda_{0}^{\times}$acts transitively on the set of pairs $\left(\alpha_{0}, \beta_{0}\right)$ of generators of $\Lambda_{0}^{\times}$. Further Aut $\Lambda_{\mathbb{Q}}^{\times}$is naturally identified with $\bar{\Gamma}_{1}:=\Gamma_{1} /\{ \pm 1\} \cong \mathbb{S}_{4}$, where the action of $\bar{\Gamma}_{1}$ on $\Lambda_{0}^{\times}$is induced by the natural inclusion $\Lambda_{0}^{\times} \cong \Gamma_{1}$ in $H^{\times}$ as a normal subgroup. Since $\Gamma_{1}$ preserves $\Lambda_{\mathfrak{z}}$ (cf. Table 2), after transforming $G$ by some element of $\bar{\Gamma}_{1}$ we may further assume that $(\alpha, \beta)=(i, j)$. Thus we have shown that $G$ is conjugate in $A(\mathbb{T})$ to one of the subgroups

$$
G_{\omega}:=\langle i,(j ;(1+\omega) / 2)\rangle, \omega=i, j, \text { or } k,
$$

where $G_{i}=Q_{0}$. Hence it remains to show that these $G_{\omega}$ are conjugate in $A\left(\mathbb{T}^{\top}\right)$ to one another. For this purpose we use the following formulae; for any $\mu \in \Gamma_{1}$ with its image $\bar{\mu}$ in $\bar{\Gamma}_{1} \subseteq A\left(\mathbb{T}^{\prime}\right)$ and for any $(\lambda ; r) \in A(\mathbb{T})$ we have

$$
\bar{\mu}(\lambda ; r) \bar{\mu}^{-1}=\left(\mu \lambda \mu^{-1} ; \mu r \mu^{-1}\right) ;
$$

if $\mu_{i}=(1+i) / \sqrt{2}$ we have

$$
\mu_{i} j \mu_{i}^{-1}=k \quad \text { and } \quad \mu_{i} k \mu_{i}^{-1}=-j .
$$

Indeed, from (20) and (21) together with analogous formulae we see 
that $\bar{\mu}_{i} G_{j} \bar{\mu}_{i}^{-1}=G_{k}$ and that if $\mu_{j}=(1+j) / \sqrt{2}$ and $\hat{t}_{*}$ is the translation by $t / 2$, we have $\bar{\mu}_{j} \hat{t}_{*} G_{i}\left(\bar{\mu}_{j} \hat{t}_{*}\right)^{-1}=G_{j}$.

Lemma 3.23. Let $(T, G)$ be any special pair with $G \cong \mathfrak{I}$ and Fix $G=\emptyset$. Let $T$ be the underlying real torus. Then $G$ is conjugate in $A(\mathbb{T})$ to $T_{0}$ with respect to the representation $\mathbb{T}=\mathbb{H} / \Lambda_{\mathfrak{x}}$ of $\mathbb{T}$.

Proof. Let $G_{1}$ be the unique normal subgroup of $G$ isomorphic to $\mathfrak{Q}$. Then by Lemma 3.20 Fix $G_{1}=\emptyset$, too. Then by Lemma $3.22 G_{1}$ is conjugate in $A(T)$ to $Q_{0}$; we may thus assume that $G_{1}=Q_{0}$. Moreover by Theorem 3.11 we may further assume that the image of $G$ in Aut $T$ is just $\Lambda_{\nwarrow}^{\times}$. Thus there exists a unique element $r \in H^{\times}$ such that $(t ; r) \in G$ so that $G=\left\langle Q_{0},(t ; r)\right\rangle$, where $t=(1+i+j+k) / 2$. Let $\tilde{t}=(t ; r)$. Then $\tilde{t}$ must satisfy the conditions

$$
\tilde{t}^{3}=-1 \quad \text { and } \quad \tilde{t}^{-1} i \tilde{t}=(k ; a), a=(1+i) / 2 .
$$

Computation then shows that these conditions are satisfied if and only if $r=s / 2+r^{\prime}$ for some $r^{\prime} \in \mathrm{Fix} \Lambda_{\mathfrak{Q}}^{\times}=\{(1+\omega) / 2 ; \omega=1, i, j, k\}$. Set $G_{\omega}=\left\langle Q_{0},(t ; s / 2+(1+\omega) / 2)\right\rangle$. Then it suffices to show that they are mutually conjugate in $A(T)$. In fact we see readily that if $\underline{\omega}_{*}$ is the translation by $(1+\omega) / 2$, then $G_{1}=\underline{i}_{*} G_{j} \underline{i}_{*}^{-1}=\underline{j}_{*} G_{k} \underline{j}_{*}^{-1}=\underline{k}_{*} G_{i} \underline{k}_{*}^{-1}$.

Let $G_{0}=Q_{0}\left(\operatorname{resp} . T_{0}\right)$ and $\mathscr{S}=\mathfrak{Q}(\operatorname{resp} . \mathfrak{I})$. Let $N_{0}(\operatorname{resp} . N)$ be the normalizer of $G_{0}$ (resp. $\Lambda_{\mathbb{E}}^{\times}$) in $A(\mathbb{T})$ (resp. Aut $\mathbb{T}$ ). Then we have $N \supseteqq \Lambda_{\nwarrow}^{\times} \supseteqq \Lambda_{\mathfrak{\Omega}}^{\times}$and if we set $\bar{N}=N / \Lambda_{\mathfrak{x}}^{\times}, \bar{N} \cong \bar{\Gamma}_{\nwarrow} \cong \widetilde{S}_{4}$ (cf. the proof of Proposition 2.19). Now $w$ induces the natural isomorphism $G_{0} \rightarrow \Lambda_{\circledast}^{\times}$ and hence the homomorphism $w_{0}: N_{0} \rightarrow N$. Let $\bar{N}_{0}$ be the image of $w_{0}\left(N_{0}\right)$ in $\bar{N}=\bar{\Gamma}_{q}$.

Lemma 3.24. In the above notations $\bar{N}_{0}$ coincides with the subgroup $\bar{\Gamma}_{0}$ of $\bar{\Gamma}_{\mathfrak{x}}$ defined after (18), (where $G_{0}=Q_{0}$ or $T_{0}$ ).

Proof. For $\mu \in \Lambda_{\mathfrak{x}}^{\times}$let $R_{\mu} \in$ Aut $_{z} \Lambda_{\mathfrak{x}}$ be the right multiplication by $\mu$. Then for $x=\left(R_{\mu} ; v\right), v \in T$, and $y=(\alpha ; r) \in G_{0}, \alpha \neq-1$, we have $x^{-1} y x=\left(\alpha ;((\alpha-1) v+r) \mu^{-1}\right)$. Hence $x^{-1} y x \in G_{0}$ if and only if $x^{-1} y x=y$, and the latter is equivalent to the equality

$$
(\alpha-1) v=r(\mu-1), \quad y=(\alpha ; r) \in G_{0} .
$$


For $y=(i ; 0) \in G_{0}$ (22) is equivalent to the condition that $v \in$ Fix $i=$ Fix $\left.Q_{0}^{\prime}{ }^{*}\right)$ Further, if $v \in$ Fix $Q_{0}^{\prime}$, then for $y=(j ; a) \in G_{0}(22)$ is equivalent to the condition; $a \mu=a$. This implies that $x=\left(R_{\mu} ; v\right)$ normalizes $Q_{0}$ if and only if $v \in$ Fix $Q_{0}^{\prime}$ and $a \mu=a$. These are certainly satisfied by $x_{i}:=\left(R_{i},(1+i) / 2\right)$ and $x_{j}:=\left(R_{j} ;(1+j) / 2\right)$. Moreover they satisfy (22) for $y=(t ; s / 2) \in T_{0}$. Therefore $x_{i}, x_{j}$ normalizes $Q_{0}$ and $T_{0}$. On the other hand, from (20) and (21) we see readily that $\hat{\mu}_{i}:=\left(\mu_{i} ; a\right)$ normalizes $Q_{0} ;$ moreover direct computation shows that

$$
\hat{\mu}_{i}(t ; s / 2) \hat{\mu}_{i}^{-1}=-(k ; a)(t ; s / 2)^{2},
$$

which implies that $\hat{\mu}_{i}$ also normalizes $T_{0}$.

Now the images of $x_{i}, x_{j}, \hat{\mu}_{i}$ in $\bar{N}$ generate $\bar{\Gamma}_{0}$ so that $\bar{\Gamma}_{0} \subseteq \bar{N}_{0}$ 。 Suppose that $\bar{\Gamma}_{0} \neq \bar{N}_{0}$. Since $\bar{\Gamma}_{0} \cong \Im_{4}$ is maximal in $\bar{N} \cong \Im_{4}$ we then have $\bar{N}=\bar{N}_{0}$, and hence $w_{0}: N_{0} \rightarrow N$ is surjective. Therefore we can find an element of $N_{0}$ of the form $\left(R_{t} ; r\right)$ for some $r \in \mathbb{T}$ (cf. Table 2). Then $\left(R_{t} ; r\right)$ must normalize $Q_{0}$. By what we have seen above this implies that $a t=a$, which is a contradiction because $a t=(1+j) / 2$ 。 Hence $\bar{N}_{0}=\bar{\Gamma}_{0}$.

Proof of Theorem 3.17. The first assertion follows from 4) of Lemma 3.20. Let $(T, G)$ be a special pair with $G \cong \cong$ and Fix $G=\emptyset$. Let $\mathbb{T}$ be the underlying real torus. By Lemma 3.22 there exists an element $u \in A(\mathbb{T})$ such that $u G u^{-1}=Q_{0}$ in $A\left(\mathbb{T}^{v}\right)$. Write $T=E / \Lambda$ and $\mathbb{T}=\mathbb{H} / \Lambda_{\mathfrak{\tau}}$. Let $u_{*}: E \rightarrow \mathbb{H}$ be the real linear isomorphism induced by $u$ so that $u_{*}$ puts a complex structure $J$ on $\mathbb{H} \cong \mathbb{R}^{4}$ which commutes with the action of $Q_{0}$, and hence of $\Lambda_{0}^{\times}$, on $\mathbb{T}$. By Theorem 3.11 this then implies that $J=J(q)$ for some $q \in X$. Then we have $(T, G) \cong$ $\left(T_{q}, H_{q}\right)$. This proves the second assertion for $Q_{0}$ 。

By construction, for any $q, q^{\prime} \in X$ an affine isomorphism of $\left(T_{q}, \hat{H}_{q}\right)$ and $\left(T_{q^{\prime}}, \hat{H}_{q^{\prime}}\right)$ is identified with an element $v$ of $A(\mathbb{T})$ which normalizes $Q_{0} \subseteq A(\mathbb{T})$ and which sends $J(q)$ to $J\left(q^{\prime}\right)$, i。 e., $v_{*} J(q) v_{*}^{-1}=J\left(q^{\prime}\right)$, where $v_{*}=w(v)_{R}$. Since the action of the normalizer $N_{0}$ of $Q_{0}$ on $X$ factors through its image $\bar{N}_{0}$ in $\bar{N}=N / \Lambda_{\Sigma}^{\times}$, the last assertion follows from Lemma 3.24 in case of $Q_{0}$. The case of $T_{0}$ is shown analogously by using Lemma 3.23 instead of Lemma 3.22.

*) $\mathfrak{a}_{0}^{\prime}:=w\left(\mathfrak{Q}_{0}\right)=\Lambda_{\mathbb{0}}^{x}(\mathrm{cf}$. Lemma 3.20$)$ 。 


\section{§4. Non-Special Pairs}

4.1. In this section we shall classify non-special pairs $(T, G)$. The classification will be given in Tables 6 through 11 below, which are arranged as follows;

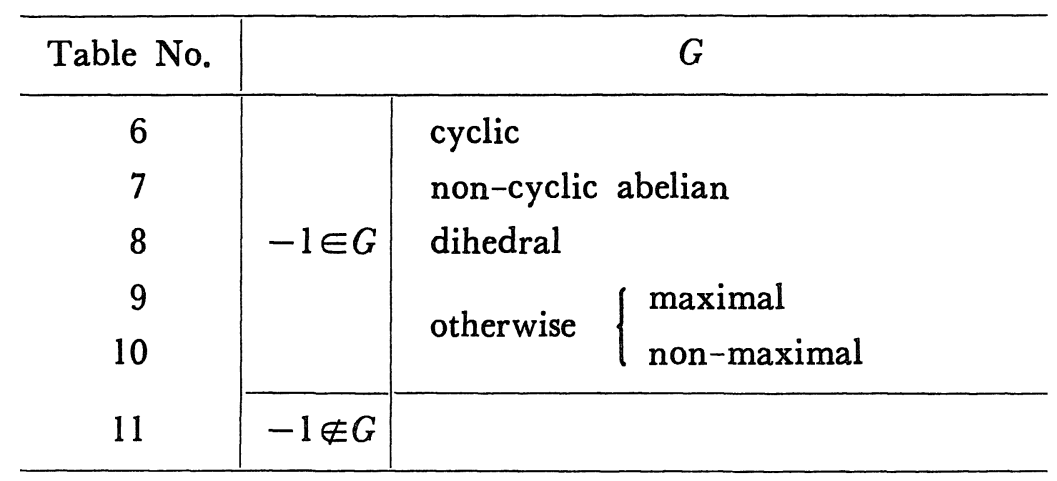

Here "maximal" means that $G$ is a maximal finite subgroup of Aut $T$. The proofs will be given after the presentation of all the tables.

In order to present the tables we shall use, besides the notations listed in Section 1, the following notations and conventions: 1. $E$ and $E^{\prime}$ will denote elliptic curves in general, considered as complex tori of dimension 1. Let $K=\operatorname{End}_{0} E$. Then either $K=\mathbb{Q}$ or $K$ is an imaginary quadratic field. Let $\mathfrak{o}$ be the maximal order of $K$. Then any element of Aut $E$ is given by a unique element of $\mathfrak{o}^{\times}$and similarly any element of Aut $(E \times E)$ will be given by a unique matrix in $\mathrm{GL}_{2}(\mathfrak{D})$. 2. Let $H$ be a finite abelian group. Let $u: H \rightarrow E, u^{\prime}: H \rightarrow E^{\prime}$ be embeddings as groups. Then we set

$$
E Y_{H} E^{\prime}=\left(E \times E^{\prime}\right) /\left(u \times u^{\prime}\right)(H),
$$

where $u$, and $u^{\prime}$ are specified each time. Let $\mathfrak{o}^{\prime}$ be the maximal order of $\operatorname{End}_{0} E^{\prime}$. Let $(a, b) \in \mathfrak{o}^{\times} \times \mathfrak{o}^{\prime \times}$. Suppose that $u(H)$ and $u^{\prime}(H)$ are invariant by $a$ and $b$ respectively. Then $(a, b)$ naturally descends to an automorphism of $E Y_{H} E^{\prime}$, which we shall again denote by the same letter $(a, b)$. 3. For $m=2,3$ the fixed point group $F\left(e_{2 m}\right)$ of $e_{2 m} \in \mathfrak{D}^{\times}$ on $E_{e_{2 m}}$ is isomorphic to $\mathfrak{E}_{m}$. Then we shall denote by $u_{m}: \mathbb{E}_{m} \rightarrow E_{e_{2 m}}$ any embedding induced by an isomorphism $\mathfrak{夭}_{m} \cong F\left(e_{2 m}\right)$. 4. (S) will denote the abstract group isomorphic to $G$. 
4. 2. With these notations we can now give Tables 6,7 , and 8 successively. In the form of a theorem we have:

Theorem 4.1. Let $(T, G)$ be a non-special pair with $-1 \in G$. Suppose that $G$ is either abelian or dihedral. Then $(T, G)$ is isomorphic to one of the pairs in Tables 6, 7, and 8.

Table $6 \quad G$ cyclic, $-1 \in G$

\begin{tabular}{|c|c|c|c|}
\hline & (5) & $T$ & $G$ \\
\hline 1 & $\mathfrak{E}_{4}$ & $E_{i} \times E_{i}$ & $\langle(i, i)\rangle$ \\
\hline $\begin{array}{l}2 \\
2^{\prime}\end{array}$ & $\mathfrak{夭}_{6}$ & $\begin{array}{l}E \times E_{\rho} \\
E Y_{\mathbb{*}_{3}} E_{\rho}\end{array}$ & $\langle(-1, \rho)\rangle$ \\
\hline 3 & & $E_{\rho} \times E_{\rho}$ & $\langle(\rho, \rho)\rangle$ \\
\hline $\begin{array}{l}4 \\
5\end{array}$ & $\mathfrak{E}_{8}$ & $\begin{array}{l}E_{i} \times E_{i} \\
E_{\sqrt{2 \bar{i}}} \times E_{\sqrt{2 \overline{2}}}\end{array}$ & $\begin{array}{l}\langle[i, 1]\rangle \\
\left\langle\left(\begin{array}{cr}2 i & -1 \\
-1 & 0\end{array}\right)\right\rangle\end{array}$ \\
\hline 6 & $\mathfrak{夭}_{10}$ & $T_{5}$ & $\left\langle\left(-\zeta,-\zeta^{2}\right)\right\rangle$ \\
\hline $\begin{array}{l}7 \\
8\end{array}$ & $\mathfrak{E}_{12}$ & $\begin{array}{l}E_{i} \times E_{i} \\
E_{\rho} \times E_{\rho}\end{array}$ & $\begin{array}{l}\left\langle\left(\begin{array}{rr}0 & 1 \\
-1 & -1\end{array}\right)\right\rangle \times\langle(i, i)\rangle \\
\left\langle\left(\rho^{2}, \rho^{2}\right),[1,-1]\right\rangle\end{array}$ \\
\hline
\end{tabular}

In $2^{\prime} u: \mathfrak{\mho}_{3} \rightarrow E$ is any embedding and $u^{\prime}: C_{3} \rightarrow E_{\rho}$ is $u_{3}$. In $6 T_{5}$ is the simple abelian variety with period matrix

$$
\left(\begin{array}{llll}
1 & \zeta & \zeta^{2} & \zeta^{3} \\
1 & \zeta^{2} & \zeta^{4} & \zeta
\end{array}\right), \quad \zeta=e_{5} .
$$

The moduli space of the pairs in 2 and $2^{\prime}$ are given respectively by $\mathrm{SL}_{2}(\mathbb{Z}) \backslash \mathfrak{E}$ and $\Gamma_{0}(3) \backslash \mathfrak{E} \cong \mathbb{C}^{*}$.

Table $7 \quad G$ abelian, but not cyclic, $-1 \in G$

\begin{tabular}{|c|c|c|c|}
\hline No. & \&S & $T$ & $G$ \\
\hline 1 & & $E \times E^{\prime}$ & \\
$1^{\prime}$ & $\mathfrak{F}_{2} \times \mathfrak{E}_{2}$ & $E Y_{\mathbb{\varepsilon}_{2}} E^{\prime}$ & $\langle(-1,1)\rangle \times\langle(1,-1)\rangle$ \\
$1^{\prime \prime}$ & & $E Y_{\mathbb{\varepsilon}_{2}^{2}} E^{\prime}$ & \\
\hline
\end{tabular}




\begin{tabular}{|c|c|c|c|}
\hline $\begin{array}{l}2 \\
2^{\prime}\end{array}$ & $\sigma \times \sigma$ & $\begin{array}{l}E \times E_{i} \\
E Y_{\mathbb{E}_{2}} E_{i}\end{array}$ & $\langle(-1,1)\rangle \times\langle(1, i)\rangle$ \\
\hline $\begin{array}{l}3 \\
3^{\prime}\end{array}$ & $\mathbb{C}_{2} \wedge_{4}$ & $E_{i} \times E_{i}$ & $\begin{array}{l}\langle(1,-1)\rangle \times\langle(i, i)\rangle \\
\left\langle\left(\begin{array}{rr}1 & 0 \\
1+i & -1\end{array}\right)\right\rangle \times\langle(i, i)\rangle \\
\left\langle\left(\begin{array}{rr}1 & 0 \\
1 & -1\end{array}\right)\right\rangle \times\langle(i, i)\rangle\end{array}$ \\
\hline 4 & \multirow{3}{*}{$\mathfrak{E}_{2} \times \mathfrak{F}_{6}$} & $E \times E_{\rho}$ & $\langle(-1,1)\rangle \times\langle(1, \rho)\rangle$ \\
\hline $\begin{array}{l}5 \\
5^{\prime}\end{array}$ & & $E_{\rho} \times E_{\rho}$ & 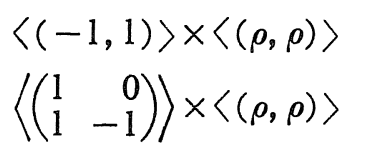 \\
\hline $\begin{array}{l}6 \\
6^{\prime}\end{array}$ & & $\begin{array}{l}E_{\rho} \times E_{\rho} \\
E_{\rho} Y_{\mathbb{\varepsilon}_{2}^{2}} E_{\rho}\end{array}$ & $\langle(-1,1)\rangle \times\left\langle\left(\rho, \rho^{2}\right)\right\rangle$ \\
\hline $\begin{array}{l}7 \\
7^{\prime}\end{array}$ & $\mathfrak{E}_{3} \times \mathfrak{E}_{6}$ & $E_{\rho} \times E_{\rho}$ & $\begin{array}{l}\left\langle\left(\rho^{2}, \rho^{-2}\right)\right\rangle \times\langle(\rho, \rho)\rangle \\
\left\langle\left(\begin{array}{rr}0 & 1 \\
-1 & -1\end{array}\right)\right\rangle \times\langle(\rho, \rho)\rangle\end{array}$ \\
\hline $\begin{array}{l}8 \\
8^{\prime}\end{array}$ & $\mathfrak{夭}_{4} \times \mathfrak{夭}_{4}$ & $E_{i} \times E_{i}$ & $\begin{array}{l}\langle(i, 1)\rangle \times\langle(1, i)\rangle \\
\left\langle\left(\begin{array}{ll}i & 0 \\
i & 1\end{array}\right)\right\rangle \times\left\langle\left(\begin{array}{rr}1 & 0 \\
-i & i\end{array}\right)\right\rangle\end{array}$ \\
\hline 9 & $\mathfrak{夭}_{6} \times \mathfrak{夭}_{6}$ & $E_{\rho} \times E_{\rho}$ & $\langle(\rho, 1)\rangle \times\langle(1, \rho)\rangle$ \\
\hline 10 & $\mathfrak{夭}_{4} \times \mathfrak{\mathfrak { E }}_{6}$ & $E_{i} \times E_{\rho}$ & $\langle(i, 1)\rangle \times\langle(1, \rho)\rangle$ \\
\hline
\end{tabular}

In $2^{\prime} u^{\prime}=u_{i}$, and in $6^{\prime} u_{2}=\bar{u}_{1}$, where ${ }^{-}$denotes the complex conjugation considered as a real analytic automorphism of $E_{i}=\boldsymbol{C} /(\boldsymbol{Z}+\mathbb{Z} i)$. Further $u$ and $u^{\prime}$ in $1^{\prime}$ and $1^{\prime \prime}$ and $u$ in $2^{\prime}$ are arbitrary. The moduli spaces for $1,1^{\prime}, 1^{\prime \prime}, 2,2^{\prime}, 4$ are respectively $\mathbb{C}^{2}, \boldsymbol{C} \times \mathbb{C}^{*}, \boldsymbol{C}^{* 2} / \widetilde{S}_{3}, \mathbb{C}, \mathbb{C}^{*}, \mathbb{C}$ (cf. Table 4). In particular they are all connected. Note that 3, 3', $3^{\prime \prime}$ (resp. 7, 7') implies that the number of conjugacy classes of elements of order 2 (resp. 3) in $\mathrm{GL}_{2}\left(R_{2}\right)$ (resp. $\mathrm{GL}_{2}\left(R_{3}\right)$ ) with eigenvalues $\{+1,-1\}$ (resp. $\left\{\rho^{2}, \rho^{-2}\right\}$ ) equals 3 (resp. 2). (Recall that $R_{m}=\mathbb{Z}\left[e_{2 m}\right]$.) 
Table $8 \quad G$ dihedral, $-1 \in G$

\begin{tabular}{|c|c|c|c|}
\hline No. & (B) & $T$ & $G$ \\
\hline 1 & \multirow{3}{*}{$\mathfrak{D}_{4}$} & $E \times E$ & \multirow{3}{*}{$\hat{D}_{4}:=\langle[1,-1], \quad[1,1]\rangle$} \\
\hline $1^{\prime}$ & & $E_{\Subset} Y_{2} E$ & \\
\hline $1^{\prime \prime}$ & & $E Y_{{ }_{\mathrm{e}_{2}^{2}} E} E$ & \\
\hline 2 & \multirow{2}{*}{$\mathfrak{D}_{6}$} & $E \times E$ & \multirow{2}{*}{$\hat{D}_{6}:=\left\langle\left(\begin{array}{rr}0 & -1 \\
1 & 1\end{array}\right),[1,1]\right\rangle$} \\
\hline $2^{\prime}$ & & $E Y_{\varepsilon_{3}} E$ & \\
\hline
\end{tabular}

In $1^{\prime}, 1^{\prime \prime}, 2^{\prime}$ the only condition imposed on $u, u^{\prime}$ is that $u=u^{\prime}$, i. e, $u \times u^{\prime}: H \rightarrow E \times E$ is a diagonal embedding. The automorphisms $[1, \pm 1]$ and $\left(\begin{array}{rr}0 & -1 \\ 1 & 1\end{array}\right)$ on $E \times E$ then descend to unique automorphisms of the corresponding quotients, which is still denoted by the same letter above. In 1, 2 the moduli spaces are isomorphic to $\mathbb{C}$ and in $1^{\prime}, 1^{\prime \prime}$, $2^{\prime}$ they are isomorphic to $\mathbb{C}$ - \{points\} (cf. Table 4 and (14)).

In Tables 6-8 above, the pairs in $k, k^{\prime}$ (and $k^{\prime \prime}$ ) are characterized as having one and the same complex representation type.

4. 3. Still under the assumption that $-1 \in G$ we say that a nonspecial pair $(T, G)$ is exceptional if $G$ is neither abelian nor dihedral. Let $F=(A, \mathfrak{o}, H, M)$ be the quadruple associated to $(T, G)$. Then it is not difficult to see that $(T, G)$ is exceptional if and only if $A \cong M_{2}(K)$ for some imaginary quadratic field $K$. In particular $T$ is then a singular abelian surface. In any case it will turn out that exceptional pairs are up to isomorphisms finite in number. We call an exceptional pair $(T, G)$ maximal if there exists no finite subgroup of Aut $T$ containing $G$ properly. First we shall give the classification of maximal pairs. Let $G_{0}$ be the normal subgroup of $G$ defined by

$$
G_{0}=f^{-1}(\operatorname{SL}(E))=\{g \in G ; \operatorname{det} f(g)=1\},
$$

where $f: G \rightarrow \mathrm{GL}(E)$ is the complex representation of $G$. Then the classification is given according to the structure of $G_{0}$ as classified in Lemma 3.3.

Theorem 4.2. Let $(T, G)$ be any non-special pair with $-1 \in G$. Suppose that $(T, G)$ is exceptional and maximal in the sense defined above. Then $(T, G)$ is isomorphic to one of the pairs in the following table. 
Table $9(T, G)$ exceptional and maximal, $-1 \in G$

\begin{tabular}{|c|c|c|c|c|}
\hline No. & $G_{0}$ & $T$ & $G$ & $|G|$ \\
\hline 1 & \multirow{2}{*}{$\mathfrak{F}_{6}$} & $E_{i} \times E_{i}$ & $\left\langle\left(\begin{array}{rr}0 & 1 \\
-1 & -1\end{array}\right),\left(\begin{array}{cr}1 & i \\
i-1 & -1\end{array}\right)\right\rangle \cong G_{6,4,3}$ & 24 \\
\hline 2 & & $E_{\rho} \times E_{\rho}$ & $\left\langle\left(\rho^{2}, \rho^{2}\right)\right\rangle \times \hat{D}_{6} \cong \mathbb{E}_{3} \times \mathfrak{I}_{6}$ & 36 \\
\hline 3 & \multirow{3}{*}{$\mathfrak{Q}$} & $E_{i} \times E_{i}$ & $\langle(i, 1)\rangle \ltimes Q_{i} \cong \mathfrak{E}_{4} \ltimes \mathfrak{Q}$ & 32 \\
\hline 4 & & $E_{\sqrt{2} \overline{2} i} \times E_{\sqrt{2} i}$ & $\left\langle\left(\begin{array}{cc}1 & \sqrt{2} i \\
0 & -1\end{array}\right)\right\rangle \ltimes Q_{\sqrt{2} i} \cong \mathfrak{E}_{2} \aleph_{1} \mathfrak{Q}$ & 16 \\
\hline 5 & & $E_{\rho} \times E_{\sqrt{3} i}$ & $\left\langle\left(\begin{array}{rr}\rho & -1 \\
-2 & -\sqrt{3} i\end{array}\right)\right\rangle \ltimes Q_{\rho} \cong \mathfrak{F}_{3} \ltimes \mathfrak{Q} \cong \mathfrak{T}$ & 24 \\
\hline 6 & \multirow{3}{*}{$\mathfrak{I}$} & $E_{i} \times E_{i}$ & $\left\langle\left(\begin{array}{ll}1 & 1 \\
0 & i\end{array}\right)\right\rangle \ltimes T_{i} \cong \mathfrak{E}_{4} \ltimes \mathfrak{I}$ & 96 \\
\hline 7 & & $E_{\sqrt{2} i} \times E_{\sqrt{2} i}$ & $\left\langle\left(\begin{array}{cc}1 & 1+\sqrt{2} i \\
0 & -1\end{array}\right)\right\rangle \ltimes T_{\sqrt{2} i} \cong \mathfrak{F}_{2} \aleph_{1} \mathfrak{T}$ & 48 \\
\hline 8 & & $E_{\rho} \times E_{\rho}$ & $\langle(\rho, \rho)\rangle \times T_{\rho} \cong \mathfrak{F}_{3} \times \mathfrak{T}$ & 72 \\
\hline 9 & \multirow{3}{*}{ (2) } & $E_{i} \times E_{i}$ & $\langle(i, i)\rangle Y_{\langle-1\rangle} \hat{D}_{6} \cong \mathfrak{S}_{4} Y_{\mathbb{E}_{2}} \mathfrak{D}_{6}$ & 24 \\
\hline 10 & & $E_{\sqrt{3} i} \times E_{\sqrt{3} i}$ & $\left\langle\left(\begin{array}{cc}1 & \sqrt{3} i \\
0 & -1\end{array}\right)\right\rangle \ltimes D_{\sqrt{3} i} \cong \mathfrak{F}_{2} \aleph_{1} \supseteqq$ & 24 \\
\hline 11 & & $E_{\rho} \times E_{\rho}$ & $\langle(\rho, 1)\rangle \ltimes D_{\rho} \cong \mathbb{E}_{6} \ltimes \mathfrak{D}$ & 72 \\
\hline
\end{tabular}

As the proof will show, in 3-5 $\left(T, G_{0}\right)$ is maximal as a special pair (cf. Theorem 3.11).

Notations. 1. We choose the presentations of $\mathfrak{Q}, \mathfrak{I}$, and $\mathfrak{D}$ as abstract groups as follows;

$$
\begin{aligned}
& \mathfrak{Q}=\left\langle a, b: a^{4}=b^{4}=1, b^{-1} a b=a^{-1}\right\rangle, \\
& \mathfrak{T}=\langle\mathrm{c}\rangle \propto \mathfrak{Q}, c^{3}=1 \\
& \mathfrak{I}=\left\langle a, b: a^{4}=b^{6}=1, a^{-1} b a=b^{-1}\right\rangle .
\end{aligned}
$$

For $\mathbb{S}=\mathfrak{S}, \mathfrak{I}, \mathfrak{D}$ the groups $\mathbb{S}_{\mu}, \mu=i, \sqrt{2} i, \rho, \sqrt{3} i$, are defined according to this presentations as follows;

$$
\begin{aligned}
& Q_{i}=\langle(i,-i),[-1,1]\rangle, \\
& Q_{\sqrt{2} i}=\left\langle\left(\begin{array}{cc}
\sqrt{2} i & -1 \\
-1 & -\sqrt{2} i
\end{array}\right),\left[\begin{array}{ll}
-1, & 1]
\end{array}\right)\right. \\
& Q_{\rho}=\left\langle\left(\begin{array}{cc}
\sqrt{3} i & -1 \\
-2 & -\sqrt{3} i
\end{array}\right),\left(\begin{array}{cc}
-1 & -\rho \\
2 \rho^{-1} & 1
\end{array}\right)\right\rangle
\end{aligned}
$$




$$
\begin{aligned}
& T_{\alpha}=\left\langle\left(\begin{array}{rr}
-1 & -1 \\
1 & 0
\end{array}\right)\right\rangle \ltimes \hat{Q}_{\alpha}, \quad \alpha=i \text { or } \sqrt{2 i} \\
& T_{\rho}=\left\langle\left(\begin{array}{ll}
\rho^{2} & -1 \\
0 & \rho^{-2}
\end{array}\right)\right\rangle \ltimes \hat{Q}_{\rho},
\end{aligned}
$$

where

$$
\begin{aligned}
& \hat{Q}_{i}=\left\langle\left(\begin{array}{cc}
i & -1+i \\
0 & -i
\end{array}\right),\left(\begin{array}{cc}
-i & 0 \\
1+i & i
\end{array}\right)\right\rangle, \hat{Q}_{\rho}=\left\langle\left(\begin{array}{cc}
\rho & \rho^{2} \\
\rho^{2} & -\rho
\end{array}\right),[-1,1]\right\rangle \\
& \hat{Q}_{\sqrt{2} i}=\left\langle\left(\begin{array}{ll}
-1+\sqrt{2} i & -2 \\
-\sqrt{2} i & 1-\sqrt{2} i
\end{array}\right),\left(\begin{array}{cc}
1 & \sqrt{2} i \\
\sqrt{2} i & -1
\end{array}\right)\right\rangle, \\
& D_{i}=\left\langle\left(\begin{array}{cc}
i & -1 \\
0 & -i
\end{array}\right),\left(\begin{array}{cc}
0 & -i \\
-i & 1
\end{array}\right)\right\rangle, D_{\sqrt{3} i}=\left\langle\left(\begin{array}{cc}
-\sqrt{3} i & 1 \\
2 & \sqrt{3} i
\end{array}\right),\left(\begin{array}{cc}
2 & \sqrt{3} i \\
\sqrt{3} i & -1
\end{array}\right)\right\rangle \\
& D_{\rho}=\left\langle\left[\begin{array}{ll}
1, & -1
\end{array}\right],\left(\rho, \rho^{-1}\right)\right\rangle .
\end{aligned}
$$

2. In general $G_{m, k ; t}$ denotes the metacyclic group defined by

$$
G_{m, k ; t}=\left\langle a, u ; a^{m}=1, u^{k}=a^{t}, u^{-1} a u=a^{-1}\right\rangle .
$$

3. There are two types, say type 1 and 2 , of nontrivial semidirect product $\mathfrak{夭}_{2} \ltimes \mathfrak{Q}$ of $\mathfrak{Q}$ by $\mathfrak{夭}_{2}$. In $4 \mathfrak{夭}_{2} \ltimes_{1} \mathfrak{Q}$ means that it is of type 1 . Similar remark applies also to 7 and 10. For the exact definitions of types 1 and 2 used here see Remark 4.3 below.

4. Let $N, K, H$ be finite groups and $j_{H}: N \rightarrow H$ and $j_{K}: N \rightarrow K$ be embeddings such that the images are contained in the centers. Then we denote by $H Y_{N} K$ the quotient group $(H \times K) /\left(j_{H} \times j_{K}\right)(N)$.

Next, we classify non-maximal pairs. Let $(T, G)$ be any exceptional and non-maximal pair with $-1 \in G$. Let $G^{\prime}$ be any maximal finite subgroup of Aut $T$ containing $G$. Then $\left(T, G^{\prime}\right)$ must be isomorphic to one of the pairs in Table 9. Therefore the problem is reduced to classifying for each pair $\left(T, G^{\prime}\right)$ of Table 9 the subgroup $G$ of $G^{\prime}$ with $G_{0}^{\prime} \sqsubseteq G \sqsubseteq G^{\prime}$ and $-1 \in G$, up to conjugacy in Aut $T$. (It turns out that different pairs necessarily give rise to non-isomorphic pairs; in other words, given ( $T, G), G^{\prime}$ is unique up to isomorphisms.) In this formulation our result will be summarized in the following table. (No. in the second column will refer to the number of the pair $\left(T, G^{\prime}\right)$ in Table 9.) 
Table $10(T, G)$ exceptional and nonmaximal, $-1 \in G$

\begin{tabular}{|c|c|c|c|c|c|}
\hline No. & & $T$ & $G$ & (5) & order \\
\hline $\begin{array}{l}1 \\
2\end{array}$ & 3 & $E_{i} \times E_{i}$ & $\begin{array}{l}\langle[i, 1],(-i, i)\rangle \\
\langle(-1,1)\rangle \ltimes Q_{i}\end{array}$ & $\begin{array}{l}G_{4,4 ; 2} \\
\mathfrak{\mho}_{2} \ltimes_{2} \mathfrak{\Omega}\end{array}$ & $\begin{array}{l}16 \\
16\end{array}$ \\
\hline 3 & & & $\left\langle\left(\begin{array}{cc}1 & 1+i \\
0 & -1\end{array}\right)\right\rangle \ltimes T_{i}$ & $\mathfrak{夭}_{2} \nwarrow_{2} \mathfrak{T}$ & 48 \\
\hline 4 & 6 & $E_{i} \times E_{i}$ & $\left\langle\left(\begin{array}{cc}1 & 1+i \\
0 & -1\end{array}\right)\right\rangle \ltimes \hat{Q}_{i}$ & $\mathfrak{\mho}_{2} \ltimes_{2} \mathfrak{Q}$ & 16 \\
\hline 5 & & & $\left\langle\left(\begin{array}{ll}1 & 1 \\
0 & i\end{array}\right)\right\rangle \ltimes \hat{Q}_{i}$ & $\mathfrak{E}_{4} \ltimes \mathfrak{Q}$ & 32 \\
\hline 6 & 7 & $E_{\sqrt{2} i} \times E_{\sqrt{2} i}$ & $\left\langle\left(\begin{array}{cc}1 & 1+\sqrt{2 i} \\
0 & -1\end{array}\right)\right\rangle \ltimes \hat{Q}_{\sqrt{2} i}$ & $\mathfrak{\mho}_{2} \ltimes_{1} \mathfrak{Q}$ & 16 \\
\hline $\begin{array}{l}7 \\
8_{ \pm}\end{array}$ & 8 & $E_{\rho} \times E_{\rho}$ & $\begin{array}{c}\left\langle\left(\rho^{2}, \rho^{2}\right)\right\rangle \times \hat{Q}_{\rho} \\
\Gamma_{ \pm}\end{array}$ & $\begin{array}{c}\mathfrak{E}_{3} \times \mathfrak{Q} \\
\mathfrak{T}\end{array}$ & $\begin{array}{l}24 \\
24\end{array}$ \\
\hline $\begin{array}{r}9 \\
10 \\
11 \\
12\end{array}$ & 11 & $E_{\rho} \times E_{\rho}$ & $\begin{array}{l}\langle[\rho, \rho],[1,1]\rangle \\
\left\langle\left(\rho^{2}, \rho^{2}\right),[1,1],\left(\rho, \rho^{-1}\right)\right\rangle \\
\langle(-1,1)\rangle \times D_{\rho} \\
\left\langle\left(\rho^{2}, \rho^{2}\right)\right\rangle \times D_{\rho}\end{array}$ & $\begin{array}{l}\mathfrak{G}_{3} \times \mathfrak{D}_{4} \\
\mathfrak{E}_{3} \times \mathfrak{D}_{6} \\
\mathfrak{E}_{2} \times \mathfrak{D}_{1} \\
\mathfrak{G}_{3} \times \mathfrak{D}\end{array}$ & $\begin{array}{l}24 \\
36 \\
24 \\
36\end{array}$ \\
\hline
\end{tabular}

Remark 4.3. We explain the notations used in Tables 9 and 10 . Let $\mathfrak{\Re}=\mathfrak{Q}$, $\mathfrak{I}$, or $\mathfrak{D}$. Let $\mathfrak{夭}_{k} \perp \Re$ be a semidirect product in general defined by an embedding $j_{k}: \Im_{k} \longrightarrow$ Aut $\Omega$. (Note the natural isomorphisms Aut $\mathfrak{Q} \cong$ Aut $\mathfrak{T} \cong \mathbb{S}_{4}$ and Aut $\mathfrak{D} \cong \mathbb{C}_{2} \times \mathfrak{D}_{3}$.) Then the following hold:

1) $\mathfrak{E}_{k} \ltimes \Omega$ is up to isomorphisms independent of the choice of $j_{k}$ in the following cases; a) $\Re=\mathfrak{Q}$ or $\mathfrak{I}$ and $k=3$ or 4 , and b) $\Re=\mathfrak{D}$ and $k=3$ or 6 . Moreover $\mathfrak{C}_{3} \ltimes \mathfrak{Q} \cong \mathfrak{I}, \mathfrak{C}_{3} \ltimes \mathfrak{D} \cong \mathfrak{C}_{3} \times \mathfrak{D}$ and $\mathfrak{E}_{3} \times \mathfrak{I} \cong \mathfrak{C}_{3} \times \mathfrak{I}$.

2) Suppose that $k=2$ a a) If $\mathfrak{A}=\mathfrak{Q}$ or $\mathfrak{I}$, there exist (up to isomorphisms) two types of $\mathfrak{\Im}_{2} \ltimes \Re$ according as $\operatorname{Im} j_{2} \sqsubseteq V$ or $\operatorname{Im} j_{2} \cong V$, where $V$ is the unique normal subgroup of $\mathfrak{S}_{4}$ of order 4 . The corresponding semidirect products will then be denoted by $\mathfrak{E}_{2} \triangleright_{1} \Re$ and

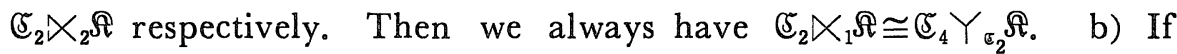
$\Re=\mathfrak{D}$, we have three types $\mathfrak{S}_{2} \ltimes_{\alpha} \mathfrak{D}, \alpha=1,2$, or 3 , according as $\operatorname{Im} j_{2}=$ $\mathfrak{夭}_{2} \times\{1\},\{1\} \times\langle\tau\rangle$, or $\langle(-1, \tau)\rangle$, where $\mathfrak{夭}_{2}=\{ \pm 1\}$ and $\tau$ is any 
element of order 2 in $\mathfrak{D}_{3}$. We see that $\mathfrak{E}_{2} \ltimes_{2} \mathfrak{D} \cong \mathfrak{C}_{4} Y_{\mathbb{\varepsilon}_{2}} \mathfrak{D}_{6}$.

4. 4. Finally we shall consider nonspecial pairs $(T, G)$ with $-1 \notin G$. In this case if we set $\hat{G}=\langle G,-1\rangle \cong G \times\langle-1\rangle$, then $(T, \hat{G})$ is isomorphic to one of the pairs from Tables 6 through 10. Thus the problem is to find for each pair $(T, \hat{G})$ in Table 6-10 the subgroups $G$ of $\hat{G}$ of index 2 with $-1 \notin G$, up to isomorphisms of the resulting pairs $(T, G)$. The result will be listed in the following table.

Table $11 \quad-1 \notin G$

\begin{tabular}{|c|c|c|c|}
\hline No. & (S) & No. for $(T, \hat{G})$ & $G$ \\
\hline 1 & $\mathfrak{S}_{2}$ & $7.1,7.1^{\prime}, 7.1^{\prime \prime}$ & $\langle(-1,1)\rangle$ \\
\hline $\begin{array}{l}2 \\
3\end{array}$ & $\mathfrak{F}_{3}$ & $\begin{array}{ll}6.2, & 6.2^{\prime} \\
6.3 & \end{array}$ & $\begin{array}{l}\left\langle\left(1, \rho^{2}\right)\right\rangle \\
\left\langle\left(\rho^{2}, \rho^{2}\right)\right\rangle\end{array}$ \\
\hline 4 & $\mathfrak{S}_{4}$ & $7.2,7.2^{\prime}$ & $\langle( \pm 1, i)\rangle$ \\
\hline 5 & $\mathfrak{S}_{5}$ & 6.6 & $\left\langle\left(\zeta, \zeta^{2}\right)\right\rangle$ \\
\hline $\begin{array}{l}6 \\
7 \\
7^{\prime} \\
8 \\
8^{\prime}\end{array}$ & $\mathfrak{\Im}_{6}$ & $\begin{array}{l}7.4 \\
7.5 \\
7.5^{\prime} \\
7.6 \\
7.6^{\prime}\end{array}$ & $\begin{array}{l}\langle(1, \rho)\rangle \times\left\langle\left(-1, \rho^{2}\right)\right\rangle \\
\langle(\rho,-\rho)\rangle \\
\langle \pm \rho A\rangle \\
\left\langle\left(\rho, \rho^{2}\right)\right\rangle \\
\left\langle \pm\left(\rho, \rho^{2}\right)\right\rangle\end{array}$ \\
\hline 9 & $\mathfrak{\Im}_{12}$ & 7.10 & $\left\langle\left(i, \pm \rho^{2}\right)\right\rangle$ \\
\hline $\begin{array}{l}10 \\
10^{\prime} \\
11\end{array}$ & 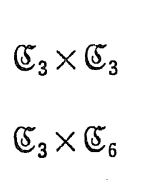 & $\begin{array}{l}7.7 \\
7.7^{\prime} \\
7.9\end{array}$ & $\begin{array}{l}\left\langle\left(\rho^{2}, \rho^{-2}\right)\right\rangle \times\left\langle\left(\rho^{2}, \rho^{2}\right)\right\rangle \\
\langle B\rangle \times\left\langle\left(\rho^{2}, \rho^{2}\right)\right\rangle \\
\left\langle\left(\rho^{2}, 1\right)\right\rangle \times\langle(1, \rho)\rangle\end{array}$ \\
\hline $\begin{array}{l}12 \\
12^{\prime}\end{array}$ & $\mathfrak{D}_{3}$ & $\begin{array}{l}8.2 \\
8.2^{\prime}\end{array}$ & $\begin{array}{l}\hat{D}_{3}^{+} \\
\hat{D}_{3}\end{array}$ \\
\hline $\begin{array}{l}13 \\
13^{\prime}\end{array}$ & $\mathfrak{E}_{3} \times \mathfrak{D}_{3}$ & $\begin{array}{l}9.2 \\
9.9\end{array}$ & $\begin{array}{ll}\left\langle\left(\rho^{2},\right.\right. & \left.\left.\rho^{2}\right)\right\rangle \times \hat{D}_{3}^{+} \\
\left\langle\left(\rho^{2},\right.\right. & \left.\left.\rho^{2}\right)\right\rangle \times \hat{D}_{3}\end{array}$ \\
\hline
\end{tabular}


Here we set $A=\left(\begin{array}{rr}1 & 0 \\ 1 & -1\end{array}\right), B=\left(\begin{array}{rr}0 & 1 \\ -1 & -1\end{array}\right)$ and $\hat{D}_{3}^{+}=\langle B, \pm[1,1]\rangle . \hat{D}_{3}^{+}$ are subgroups of $\hat{D}_{6}$ isomorphic to $\mathfrak{D}_{3}$. In $4\langle( \pm 1, i)\rangle$ denotes the two groups $\langle(1, i)\rangle$ or $\langle(-1, i)\rangle$. Similar remarks apply also to Nos. $7^{\prime}, 8^{\prime}, 9,12$ and 13.

4. 5. Proof of Theorem 4. 1. Let $F=(A, \mathfrak{o}, H, M)$ be the quadruple in 2 associated to $(T, G)$. If $G$ is abelian, then $A$ is commutative so that $F$ falls under $1-11$ of Table 1 . If $G$ is dihedral, then $A$ is isomorphic to $M_{2}(\boldsymbol{Q})$; indeed, any noncommutative semisimple factors of the group ring of $\mathscr{D}_{k}$ over $\boldsymbol{Q}$ is isomorphic to $M_{2}(\boldsymbol{Q})$. Hence $F$ falls under 15 or 16 of Table 1 . In either case $(T, G)$ corresponds to a unique point of the moduli space $\mathscr{M}_{(H)}$. On the other hand, the pairs listed in Tables 6-8 are precisely those pairs which are constructed from the quadruples in $1-11$ and 15,16 of Table 1 by the construction in 2.1 and 2.2 (cf. (2) (3)); (cf. the proof of Proposition 3.13). The theorem follows.

Proof of Theorem 4.2. Let $K$ be a normal subgroup of $G$. Let $H$ be the normalizer of $K$ in Aut $T$. If $H$ is finite, then from the maximality of $(T, G)$ we must have $G=H$. Let $F=\left(A\right.$, o $\left., H^{\prime}, M\right)$ be the quadruple associated to the pair $(T, K)$. Assume that $\operatorname{rank}_{Q} A \leqq 4$. Then we may assume that $F$ is one of the quadruples in Table 1 . Let $p$ be any point of $W_{F}$ such that $\left(T_{p}, G_{p}\right) \cong(T, K)$. Then with respect to the natural action of the normalizer $N_{F}$ of $F$ on $W_{F}$ the stabilizer $N_{p}$ at $p$ is naturally isomorphic to $H$ (cf. the end of 2.1).

Now apply this remark to $K=G_{0}$ when $G_{0}$ is non-cyclic. By Lemma 3.4 $(T, K)$ satisfies the above condition that $\operatorname{rank}_{Q} A \leqq 4$. In this case by Proposition 2.15 and Lemma 2.16 we see that $N_{F}$ itself is finite and hence that $G$ is the normalizer of $G_{0}$ in Aut $T$. In particular $\left(T, G_{0}\right)$ also is maximal as a special pair. Since $G \neq G_{0}$, $(T, G)$ must be isomorphic to one of the pairs $\left(T_{\alpha}, G_{\alpha}\right)$ in Proposition 3.13 (cf. Remark 3.14). On the other hand, these pairs $\left(T_{\alpha}, G_{\alpha}\right)$ are precisely the pairs listed in 3-11 of the table. (Explicit description of $G_{\alpha}$ as in the table can be obtained by using the $\mathbb{Z}$-basis of $\Lambda_{\theta}$ mentioned in the proof of Proposition 3.13 (cf. Remark 3.14).) Thus it remains to treat the case where $G_{0}$ is cyclic. For this purpose we shall prove successively the three lemmas, Lemmas $4.4,4.5$, and 4.6 
below, from which the theorem clearly follows.

Lemma 4. 4. Let $(T, G)$ be a pair with $-1 \in G$. Let $2 n$ be the order of $G$ and $m$ the order of $G_{0}$. Suppose that $G_{0}$ is cyclic and $G$ is non-abelian and non-dihedral. Then either 1) $G$ is isomorphic to $\mathfrak{\mho}_{3} \times \mathfrak{D}_{m}$, $m=4,6$, or 2) $n=8$ or 12 and $G$ contains an element of order $n$ with eigenvalues $\left(e_{n},-e_{n}\right)$.

Proof. Fixing an isomorphism $E \cong \mathbb{C}^{2}$ we consider $G$ as a subgroup of $\mathrm{GL}_{2}(\mathbb{C})$ via complex representation. Fix a generator $a$ of $G_{0}$ and an element $b$ of $G$ whose image in $G / G_{0}$ generates the cyclic group $G / G_{0} \subseteq C^{*}$. Let $l$ be the order of $b$. We know by Lemma 3.2 that $\varphi(l)=2$ or 4 . Up to conjugation in $\mathrm{GL}_{2}(\mathbb{C})$ we may assume that a is of the diagonal form $\left(\zeta_{m}, \zeta_{m}^{-1}\right)$ for some primitive $m$-th root of unity $\zeta_{m}$. Since $G$ is nonabelian, $b$ must then be of the form $\left[d, d^{\prime}\right]$ for some $d, d^{\prime} \in C^{*}$ (cf. Notation). In particular $b^{2}$ is a scalar matrix, while $b$ is not. Then in view of the possible eigenvalues of $b$ when $\varphi(l)=4$ (cf. (15)), from this and the fact that $b \notin G_{0}$ we see readily that $l \neq 3,4,5,10$ and that if $l=2,6,8$, or 12 , then eigenvalues of $b$ are $\pm \zeta_{l}$. Then by replacing $b$ by some of its power, we may assume that $\zeta_{l}=e_{l}$.

Now if $l=12$, then $b^{3} \in G_{0}$ and hence the image of $b^{4}$ again generates $G / G_{0}$, which is a contradiction with what we have seen above. In case $l=2$ or $6, G$ is the nontrivial semidirect product $\langle b\rangle \ltimes G_{0}$. It follows that if $l=2, G$ is dihedral; hence this case does not occur. Similarly, if $l=6$, we see that $G$ is in case 1 ) of the lemma. So suppose that $l=8$. In this case the order of $G / G_{0}$ equals 4 so that $n=2 m$. Note on the other hand that $m=4$ or 6 as $-1 \in G$ and $G$ is nonabelian. Thus if $m=4, G$ is clearly in case 2) of the lemma. If $m=6, a b^{2}$ is of order 12 and hence $G$ again falls under the case 2).

Each case of the above lemma is divided further into two cases as follows; la) $G \cong \mathfrak{\Im}_{3} \times \mathfrak{I}_{4}$, lb) $G \cong \mathfrak{\Im}_{3} \times \mathfrak{I}_{6}$ and $\left.2 \mathrm{a}\right) \mathrm{n=8}$ and $\left.2 \mathrm{~b}\right) \mathrm{n}=12$.

Lemma 4. 5. There exists a unique (resp. exactly two) isomorphism classes of pairs in the cases 1a), 2a), 2b) (resp. 1b)).

Proof. First we consider the cases 2a), 2b). Let $c$ be any element 
of order $n$ of $G$ as in the previous lemma. By Theorem 4.1 $(T,\langle c\rangle)$ is isomorphic to the pair of Table 6, No. 4 (resp. No. 8) if $n=8$ (resp. 12). Moreover, the normalizer $N$ of $\langle c\rangle$ in Aut $T$ is of order $2 n$ (cf. the proof of Proposition 2.19). Hence we must have $G \cong N$. It follows that $(T, G)$ is up to isomorphisms unique.

Next, we consider the cases la), lb). We use the notation of the first part of the proof of Theorem 4.2. Take $K$ to be the normal subgroup of $G$ isomorphic to $D_{k}, k=4,6$. (Such a $K$ is unique.) Then $N_{p}$ is finite so that $G$ must be isomorphic to $N_{p}$; hence $\bar{N}_{p}:=N_{p} / K$ is cyclic of order 3. In fact, in this case $\bar{N}_{p}$ is identified with the stabilizer $\Gamma_{p}$ at $p \in \mathscr{S}$ in $\Gamma=\mathrm{SL}_{2}(\mathbb{Z})$ (resp. $\check{\mathfrak{o}}_{1}^{\times} \rtimes \mathfrak{F}_{2}$ ) considered $\bmod \pm 1$ if $\mathfrak{D}=M_{2}(\mathbb{Z}) \quad\left(\right.$ resp. $\left.\neq M_{2}(\mathbb{Z})\right)$. Let $L$ be the set of points of $\mathfrak{\mathcal { C }}$ whose stabilizer in $\Gamma$ is cyclic of order 6 . Let $n$ be the number of $\Gamma$-orbits in $L$. Then it suffices to show that 1) $n=1$ if $\left.\mathfrak{o}=M_{2}(\mathbb{Z}), 2\right) n=0$ if $\mathfrak{o} \neq M_{2}(\mathbb{Z})$ and $k=4$, and 3$) n=1$ if $\mathfrak{p} \neq M_{2}(\mathbb{Z})$ and $\left.k=6.1\right)$ is wellknown. For 2) and 3) one first note that since $\check{o}_{1}^{\times}$is of index 2 in $\Gamma$, we may replace $\Gamma$ by $\check{o}_{1}^{x}$. Then from $\left.(14), 1\right)$, together with the fact that the principal congruence subgroup $\Gamma(m), m>1$, has no elliptic point on $\mathfrak{S}$, we can deduce easily the desired assertion.

Lemma 4.6. Let $(T, G)$ be a pair as in Lemma 4.4. Then $(T, G)$ is maximal and is isomorphic to the pair of Table 9, No. 1 in the case 2b); it is not maximal in the cases $1 a$ ) and $2 a$ ) and is isomorphic to the pairs of Table 10, No.9 and No.1, respectively. In the case 1b) one is maximal and one is noi (cf. Lemma 4.5). The maximal one is isomorphic to the pair of Table 9, No.2 and the non-maximal one is isomorphic to the pair of Table 10, No. 10.

Proof. Let $\left(T_{\mu}, G_{\mu}\right), \mu=1,2,3$, be the pairs of Table 10, Nos. 1, 9 , and 10 respectively. Then $G_{\mu}, \mu=2,3$, are subgroups of $G^{\prime}:=$ $(\rho, 1) \ltimes D_{\rho}$. It is immediate to see that these falls under the cases 2a), la) and lb) respectively, and moreover that any subgroup of $G^{\prime}$ in $\mathrm{GL}_{2}\left(R_{3}\right) \quad\left(R_{3}=\mathbb{Z}[\rho]\right)$ which is isomorphic to $\mathfrak{S}_{3} \times \mathfrak{D}_{6}$ and containing $\left(\rho, \rho^{-1}\right)$ are conjugate to $G_{3}$ in $G^{\prime}$. It follows from Lemma 4.5 that the pairs in 2a) and la) and exactly one of the pairs in lb) are not maximal. On the other hand, we know that $\left(\begin{array}{rr}0 & -1 \\ 1 & 1\end{array}\right)$ is not conjugate to $\left(\rho, \rho^{-1}\right.$ ) in $\mathrm{GL}_{2}\left(R_{3}\right)$ (cf. Table 7 ). Hence the group of Table 9, 
No. 2 is not conjugate in $\operatorname{Aut}\left(E_{\rho} \times E_{\rho}\right)$ to $G_{3}$; hence the corresponding pair is maximal.

Finally we show the maximality of the pair $(T, G)$ in $2 \mathrm{~b})$. Let $c$ be any element of $G$ of order 12. Then $c^{3}$ has eigenvalues $\{i, i\}$. Hence by Theorem 4.1 T must be isomorphic to $E_{i} \times E_{i}$ 。 Let $G^{\prime}$ be any maximal finite subgroup of Aut $T$ containing $G$. Suppose that $G \neq G^{\prime}$. By Lemma $4.4 G_{0}^{\prime}$ is then non-cyclic. Therefore $G^{\prime}$ must coincide with the normalizer of $G_{0}^{\prime}$ and $\left(T, G_{0}^{\prime}\right)$ is maximal as a special pair (cf. the first part of the proof of Theorem 4.2). Therefore we conclude that $\left(T, G^{\prime}\right)$ is isomorphic to the pair of Table 9, No. 6 in view of the fact that the order of $G$ equals 24. From this one derives easily a contradiction. Namely $(T, G)$ is maximal as desired.

As was already mentioned, Theorem 2.4 follows from the above three lemmas. As for Table 10, it can be obtained from Table 9 by straightforward consideration. By way of illustration we consider the case of subgroups $G$ of $G^{\prime}:=\langle(\rho, \rho)\rangle \times T_{\rho} \cong \mathfrak{E}_{3} \times \mathfrak{I}$ (cf. the notation before Table 10). Let $\pi: G^{\prime} \rightarrow \mathbb{E}_{3}$ be the natural projection. Then we must have $\pi(G)=\mathbb{C}_{3}$ and $G_{0}$ is either cyclic or $\hat{Q}_{\rho} \subseteq T_{\rho}$. The cyclic case has been classified in general in the proof of Lemma 4.4; it follows that this case does not occur. Hence $G_{0}=\hat{Q}_{\rho}$. We have

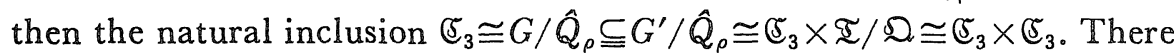
exist three subgroups of order 3 in $\mathfrak{E}_{3} \times \mathfrak{E}_{3}$ other than $\{1\} \times \mathfrak{E}_{3}$. The inverse images of these in $G^{\prime}$ are the groups of Table 10, No. 7, $8_{ \pm}$, which are not conjugate to one another in $G^{\prime}$, and $G$ coincides with one of it. Note that $\Gamma_{ \pm}$is the subgroup of $G^{\prime}$ corresponding to the (transpose of) the graph of the two non-trivial homomorphisms $f_{ \pm}: T \rightarrow \mathbb{夭}_{3}$. That $\Gamma_{ \pm}$are not conjugate in Aut $T$ follows from the fact that $\Gamma_{+} \cap \Gamma_{-}=\hat{Q}_{\rho}$ and $G^{\prime}$ is the normalizer of $\hat{Q}_{\rho}$.

The verification of Table 11 is also not difficult and will be left to the reader. From Lemma 2.20 and the above tables we conclude the following:

Theorem 4. \%. The moduli space of pairs with fixed complex and rational representation types are connected. In other words, two pairs $(T, G)$ and $\left(T^{\prime}, G^{\prime}\right)$ are equivalent under deformation if and only if their complex and rational representations are equivalent. 
4. 6. We compare our results with the results of Gottschling and Ueno in a), and with the results of Tokunaga-Yoshida in b).

a) In [8] [9] Gottschling studied fixed point manifolds and stabilizers of the standard action of $\operatorname{Sp}(2, \mathbb{Z})$ on the Siegel upper half space $\mathfrak{S}_{2}$ of degree 2. Later Ueno [25] refined his results by obtaining the classification of elements of finite order of $\operatorname{Sp}(2, \boldsymbol{Z})$ up to conjugacy. These are regarded as the classification of automorphism groups of principally polarized abelian surfaces in terms of their period matrices. A comparison of their results and ours will be given in the following:

Theorem 4.8. Let $(T, G)$ be a pair. Suppose that there exists a principal polarization on $T$ such that $G$ is the full automorphism group of the resulting principally polarized abelian surface. Then $(T, G)$ is isomorphic to one of the pairs in the following table.

Table 12

\begin{tabular}{|c|c|c|c|c|c|}
\hline No. & $T$ & \&8 & $\begin{array}{c}\text { Table } \\
\text { No. }\end{array}$ & $Z$ & $C$ \\
\hline $\begin{array}{l}1 \\
1^{\prime}\end{array}$ & $\begin{array}{l}E \times E^{\prime} \\
E Y_{\mathbb{\epsilon}_{2}^{2}} E^{\prime}\end{array}$ & $\mid \mathfrak{F}_{2} \times \mathfrak{F}_{2}$ & $\begin{array}{l}7.1 \\
7.1^{\prime \prime}\end{array}$ & $\begin{array}{l}\left(\begin{array}{ll}z & 0 \\
0 & z^{\prime}\end{array}\right) \\
\left(\begin{array}{cc}z & 1 / 2 \\
1 / 2 & z^{\prime}\end{array}\right)\end{array}$ & $y^{2}=x^{6}+\alpha x^{4}+\beta x^{2}+1$ \\
\hline $\begin{array}{l}2 \\
2^{\prime}\end{array}$ & $\begin{array}{l}E \times E \\
E Y_{\epsilon_{2}^{2}} E\end{array}$ & $\mathfrak{D}_{4}$ & $\begin{array}{l}8.1 \\
8.1^{\prime \prime}\end{array}$ & $\begin{array}{l}\left(\begin{array}{ll}z & 0 \\
0 & z\end{array}\right) \\
\left(\begin{array}{cc}z & 1 / 2 \\
1 / 2 & z\end{array}\right)\end{array}$ & $y^{2}=x\left(x^{4}+\alpha x^{2}+1\right)$ \\
\hline 3 & $E Y_{\mathfrak{a}_{3}} E$ & $\mathfrak{D}_{6}$ & 8. $2^{\prime}$ & $\left(\begin{array}{cc}z & z / 2 \\
z / 2 & z\end{array}\right)$ & $y^{2}=x^{6}+\alpha x^{3}+1$ \\
\hline 4 & $E \times E_{i}$ & $\mathfrak{\mho}_{2} \times \mathfrak{\mho}_{4}$ & 7.2 & $\left(\begin{array}{ll}z & 0 \\
0 & i\end{array}\right)$ & \\
\hline 5 & $E \times E_{\rho}$ & $\mathfrak{\Im}_{2} \times \mathfrak{夭}_{6}$ & 7.4 & $\left(\begin{array}{ll}z & 0 \\
0 & \rho\end{array}\right)$ & \\
\hline 6 & $E_{i} \times E_{i}$ & $\mathfrak{s}_{4} \ltimes \mathfrak{Q}$ & 9.3 & $\left(\begin{array}{ll}i & 0 \\
0 & i\end{array}\right)$ & \\
\hline 7 & $E_{\rho} \times E_{\rho}$ & $\mathfrak{\Im}_{6} \times \mathfrak{S}$ & 9.11 & $\left(\begin{array}{ll}\rho & 0 \\
0 & \rho\end{array}\right)$ & \\
\hline
\end{tabular}




\begin{tabular}{|c|c|c|c|c|c|}
\hline 8 & $E_{i} \times E_{\rho}$ & $\mathfrak{E}_{4} \times \mathfrak{E}_{6}$ & 7.10 & $\left(\begin{array}{ll}i & 0 \\
0 & \rho\end{array}\right)$ & \\
\hline 9 & $T_{5}$ & $\mathfrak{夭}_{10}$ & 6.6 & $\left(\begin{array}{cc}\zeta & \zeta+\zeta^{-2} \\
\zeta+\zeta^{-2} & -\zeta^{-1}\end{array}\right)$ & $y^{2}=x^{5}+1$ \\
\hline 10 & $E_{\sqrt{2} i} \times E$ & ${ }_{2} \ltimes_{1} \mathfrak{I}$ & 9.7 & $\left(\begin{array}{cc}\eta & (\eta-1) / 2 \\
(\eta-1) / 2 & \eta\end{array}\right)$ & $y^{2}=x\left(x^{4}+1\right)$ \\
\hline 11 & $E_{\sqrt{3} i} \times 1$ & $\Sigma_{2} \ltimes_{1} \mathfrak{D}$ & 9.10 & $(i / \sqrt{3})\left(\begin{array}{ll}2 & 1 \\
1 & 2\end{array}\right)$ & $y^{2}=x^{6}+1$ \\
\hline
\end{tabular}

Here $Z$ is the period of $T$ on $\mathfrak{S}_{2}$ determined up to conjugacy by elements of $\operatorname{Sp}(2, Z)$. Further, $z \neq z^{\prime}, z_{9} z^{\prime} \neq i, \neq \rho_{.} \zeta=e_{5}, \eta=(1+2 \sqrt{2} i) / 2$. In case $T$ is the Jacobian of a smooth curve of genus 2 we have given an inhomogeneous equation of it on the last column (cf. Namikawa-Ueno [13]). The stabilizer of $\operatorname{Sp}(2, \mathbb{Z})$ at $Z$ which is isomorphic to $G$ is given in [9].

b) Let $T$ be a complex torus of dimension 2. An element $g$ of Aut $T$ is called a reflection if it has a 1-dimensional fixed point set on $T$. In Table I of [24] Tokunaga-Yoshida gives a complete classification of those pairs $(T, G)$ for which $G$ is generated by reflections. Identification of their and our classifications are given as follows. Notations. $G(m, p, 2)$ the subgroup of $\mathrm{GL}_{2}(\mathbb{C})$ generated by the elements $\left(e_{m}, e_{m}^{-1}\right),[1,1],\left(e_{m}^{p}, 1\right) . G(m, p)$ the abstract group isomorphic to $G(m, p, 2)$. [k] the primitive finite reflection groups of $\mathrm{GL}_{2}(C)$ of Shepard-Todd number $k ;(k)$ the corresponding abstract group in $\mathrm{GL}_{2}(\mathbb{C})$ up to conjugacy. Now the classification in [24] is in terms of $G(m, p, 2)$ and $[k]$; for each of these groups the equivalence classes of lattices in $\mathbb{C}^{2}$ preserved by it are given. In the next table we give for each of these groups the corresponding pair $(T, G)$ in our classification table.

Table 13

\begin{tabular}{ll|l||ll|l}
\hline \multicolumn{1}{c|}{$G$} & \multicolumn{1}{|c|}{ Table No. } & \multicolumn{1}{|c|}{$G$} & \multicolumn{1}{c}{ Table No. } \\
\hline$G(1.2)$ & $\mathfrak{S}_{4}$ & $8.1,8.1^{\prime}, 8.1^{\prime \prime}$ & $G(4.1)$ & $\mathfrak{E}_{4} \times \mathfrak{Q}$ & $9.3,10.5$ \\
$G(4.2)$ & $\mathfrak{F}_{4} Y_{\mathbb{E}_{2}} \mathfrak{Q}$ & $10.2,10.4$ & $G(3.1)$ & $\mathfrak{E}_{3} \times \mathfrak{S}_{3}$ & $11.13,11.13^{\prime}$
\end{tabular}




\begin{tabular}{|c|c|c|c|c|c|}
\hline$G(6.2)$ & $\mathfrak{S}_{6} \times \mathfrak{D}_{3}$ & $9.2,10.10$ & $G(6.6)$ & $\mathfrak{D}_{6}$ & $8.2,8.2^{\prime}$ \\
\hline$G(6.3)$ & $\mathfrak{S}_{2} \chi_{1} \mathfrak{D}$ & $9.10,10.11$ & (4) & $\mathfrak{I}$ & $9.5,10.8$ \\
\hline$G(6.1)$ & $\mathfrak{夭}_{6} \times \mathfrak{D}$ & 9.11 & (5) & $\mathfrak{F}_{3} \times \mathfrak{T}$ & 9.8 \\
\hline$G(3.3)$ & $\mathfrak{D}_{3}$ & $11.12,11.12^{\prime}$ & (8) & $\mathfrak{E}_{4} \times \mathfrak{T}$ & 9.6 \\
\hline
\end{tabular}

In each case the complex representation is given by the corresponding subgroup $G(m, p, 2)$ or $[k]$ of $\mathrm{GL}_{2}(\boldsymbol{C})$.

\section{§5. Endomorphism Rings of $T$}

5. 1. The automorphism group of a complex torus is closely related with its endomorphism ring, the former being the unit group of the latter. In this section we summarize the structure of rational endomorphism rings $\operatorname{End}_{0} T$ of complex tori of dimension two; this will help us to get a better understanding of the automorphism groups themselves.

We distinguish three cases according as $T$ contains: 1) more than one, 2) exactly one, and 3) no, subtori of dimension one. In case 1) $T$ is isogenous to a product $E_{1} \times E_{2}$ of two elliptic curves $E_{1}$ and $E_{2} ; T \sim E_{1} \times E_{2}$. ( $\sim$ means "isogenous to", here and in what follows.) In case 2) we denote by $C^{\prime}$ the unique subtorus and by $C$ the quotient torus $T / C^{\prime}$. In case 3 ) $T$ is by definition simple. The relation with algebraic dimension $a(T)$ of $T$ is as follows: 1) $a(T)=2,2) a(T)=1$, and 3) $a(T)=2$ or 0 . (Recall that $a(T)=2$ if and only if $T$ is an abelian surface.) In case 2) the natural homomorphism $T \rightarrow C$ gives the algebraic reduction of $T$.

Theorem 5.1. Let $T$ be a complex torus of dimension 2. Then in each of the above three cases the possible types of rational endomorphism rings $\operatorname{End}_{0} T$, the values of Picard numbers $\rho(T)$, the dimension $d$ of the "moduli space" ( $c f .[7]$ for the precise definition) and the structure of Aut $T$ are given as in the following table.

Table 14

Case 1. $T \sim E \times E^{\prime}$

\begin{tabular}{|c|c|c|c|c|c|}
\hline No. & $T$ & $\operatorname{End}_{0} T$ & $\rho(T)$ & $d$ & Aut $T$ \\
\hline 1 & $E \nsim E^{\prime}$ & $K \oplus K^{\prime}$ & 2 & 2 & $\mathfrak{夭}_{m} \oplus \mho_{n}, 1 \leqq m, n \leqq 3$ \\
\hline
\end{tabular}




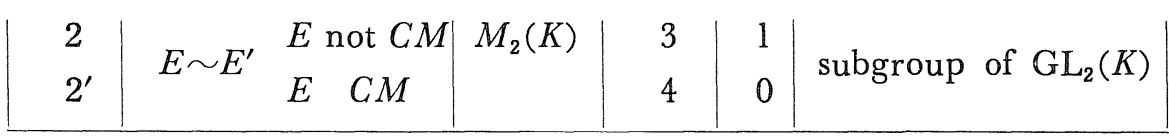

Here $K=\operatorname{End}_{0} E$ and $K^{\prime}=\operatorname{End}_{0} E^{\prime}$.

Case 2.

\begin{tabular}{|c|c|c|c|c|c|c|}
\hline No. & & $T$ & $\operatorname{End}_{0} T$ & $\rho(T)$ & $d$ & Aut $T$ \\
\hline 1 & $C+C^{\prime}$ & & $\mathbb{Q}$ & 1 & 3 & $\langle \pm 1\rangle$ \\
\hline 2 & $C_{r}$ & $C \operatorname{not} C M$ & $K\lceil V\rceil$ & 2 & 2 & $\langle \pm 1\rangle$ \\
\hline $2^{\prime}$ & $u \sim c$ & $C \quad C M$ & $(V=K)$ & 3 & 1 & $\mathfrak{夭}_{2 m}, 1 \leqq m \leqq 3$ \\
\hline
\end{tabular}

Here $K=\operatorname{End}_{0} C$. Further, in 2 or $2^{\prime}$ the elements of $V$ are considered to be square zero. Thus if $C$ is not $C M, \operatorname{End}_{0} T \cong \mathbb{Q}[X] /\left(X^{2}\right)$ and if $C$ is $C M, \operatorname{End}_{0} T \cong K[X, Y] /(X, Y)^{2}$.

Case 3. T simple

\begin{tabular}{|c|c|c|c|c|c|}
\hline No. & $a(T)$ & $\operatorname{End}_{0} T$ & $\rho(T)$ & $d$ & Aut $T$ \\
\hline 1 & \multirow{4}{*}{2} & $Q$ & 1 & 3 & $\langle \pm 1\rangle$ \\
\hline 2 & & real quadratic field & 2 & 2 & $\mathbb{Z} \oplus \mathfrak{S}_{2}$ \\
\hline 3 & & $C M$ field of degree 4 & 2 & 0 & $\mathbb{Z} \oplus \mathfrak{C}_{2 m}, m=1,5$ \\
\hline 4 & & $\begin{array}{l}\text { indefinite quaternion } \\
\text { division algebra } / \mathbb{Q}\end{array}$ & 3 & 1 & $\begin{array}{l}\text { non-abelian } \\
\text { infinite }\end{array}$ \\
\hline 5 & \multirow{5}{*}{0} & $\mathbb{Q}$ & 0 or 1 & 4 & $\langle \pm 1\rangle$ \\
\hline 6 & & $\begin{array}{l}\text { imaginary quadratic } \\
\text { field }\end{array}$ & 2 & 2 & $\mathfrak{夭}_{2 m}, 1 \leqq m \leqq 3$ \\
\hline 7 & & totally imaginary & 2 & \multirow{2}{*}{0} & $\mathbb{Z} \oplus \mathfrak{S}_{2 m}, \quad 1 \leqq m \leqq 3$ \\
\hline & & $\begin{array}{l}\text { number field of degree } \\
4 \text {, not } C M\end{array}$ & 0 & & $\mathbb{Z} \oplus \mathfrak{C}_{2}$ \\
\hline 8 & & $\begin{array}{l}\text { definite quaternion } \\
\text { algebra } / \mathbb{Q}\end{array}$ & 3 & 1 & finite \\
\hline
\end{tabular}

Here in $3, m=5$ if and only if $T \cong T_{5}$ (cf. Table 6). In 4 Aut $T$ contains a normal subgroup $\Gamma_{0}$ of index 2 which is a Fuchsian group of the first kind. Any possible finite subgroup of $\Gamma_{0}$ is isomorphic to $\mathfrak{C}_{m}$ with $m=1,2,3,4$ or 6 . Possible finite subgroups in 8 are 
those which appear in special pairs (cf. Sec. 2). In 7 if $\tilde{K}$ is the minimal Galois extension of $\operatorname{End}_{0} T$, then $\rho=2$ (resp. 0) if and only if the Galois group $\operatorname{Gal}(\tilde{K} / Q) \cong \mathfrak{D}_{4}$ (resp. $\mathfrak{A}_{4}$ or $\left.\mathfrak{S}_{4}\right)$, where $\mathfrak{A}_{4}$ is the alternating group.

For the proof of Theorem 5.1 we refer to [7] (Most of the results are special cases of those results which hold also in higher dimensions.) We only note that for abelian surfaces all the results in the above tables are well-known (cf. e. g. $[20, \S 4]$ ).

5.2. As follows from the above table, if $a(T) \leqq 1$, then Aut $T$ is infinite if and only if $a(T)=0$ and $\operatorname{End}_{0} T$ is a totally imaginary field of degree 4 which is not $C M$; conversely given any such a field $K$ one can construct a complex torus $T$ of dimension 2 with $\operatorname{End}_{0} T \cong K$ and hence with $a(T)=0$ and with Aut $T$ infinite (cf. [27] for an explicit example). If, in addition, we take $K$ with $\operatorname{Gal}(\tilde{K} / \mathbb{Q}) \cong \mathbb{S}_{4}$ or $\mathfrak{A}_{4}$, then we even have $\rho(T)=0$. In this connection, we shall show that any automorphism $g$ of infinite order of $T$ with $a(T)=0$ is never special, i. e., det $f(g) \neq 1$, where $f: \operatorname{Aut~} T \rightarrow \mathrm{GL}(E)$ is the complex representation. This is in a good contrast to the case of abelian surfaces since if $a(T)=2$ (instead of 0 ), $g^{k}$ is special for some positive integer $k$ by a theorem of Zarhin [28]. The fact is even true for $\mathrm{K} 3$ surfaces, if we make the following definition. An automorphism $g$ of a K3 surface (or complex torus) $S$ is said to be special (or symplectic) if $g^{*} \omega=\omega$ for any holomorphic 2 -form $\omega$ on $S$. (For a complex torus this definition coincides with the original one.)

Proposition 5.2. Let $S$ be a complex torus of dimension 2 or a $\mathrm{K} 3$ surface. Let $g$ be an automorphism of infinite order of $S$. Then $g$ is never special if $a(T)=0$.

Lemma 5.3. Let $S$ be as in the above proposition. Let $N_{S}$ be the Neron-Severi lattice of $S$. Then the natural homomorphism $u$ :Aut $S \rightarrow$ Aut $N_{S}$ factors through a finite group.

Proof. Since $a(S)=0, N_{S}$ is negative definite (cf. $[14,3.5]$ ). Then $u($ Aut $T$ ) is considered as a discrete subgroup of the orthogonal group. Hence it is finite. 
Proof of Proposition 5.2. By Lemma $5.3 \mathrm{~g}^{k}$ acts trivially on $N_{s}$ for some positive integer $k$. Then $g^{k}$, and hence $g$ also, act on the transcendental lattice of $S$ nontrivially since $g^{k} \neq \pm 1$. (An automorphism which acts trivially on both $N_{S}$ and $B_{S}$ is either of \pm 1 .) Then by $[14$, Th. 3.1] $g$ is not special.

Table 14 also shows that if $a(T)=0$ and $\rho(T)=3$, Aut $T$ is finite. This also admits an analogy in the case of $K 3$ surfaces. Namely we prove

Proposition 5.4. Let $S$ be a $K 3$ surface with $a(T)=0$ and $\rho(S)=19$. Then the automorphism group of $S$ is finite.

Proof. In this case the rank of the transcendental lattice $B_{S}$ of $S$ equals 3. Since $H^{2}(S, \mathbb{R})$ has signature $(3,19)$ and $N_{S}$ is negative definite, it follows that $B_{S}$ is positive definite. Hence by the same argument as in the proof of Lemma 5.3 the action of Aut $T$ on $B_{S}$ factors through a finite subgroup as well as the action on $N_{s}$. From this the proposition follows immediately.

Remark 5.5. 1) $\rho=19$ is the maximal number for the Picard number of a $K 3$ surface with $a(S) \leqq 1$. 2) The above proof also applies to a complex torus, thus giving another proof for the fact mentioned before the proposition.

5. 3. Using the structure of the endomorphism ring described above,

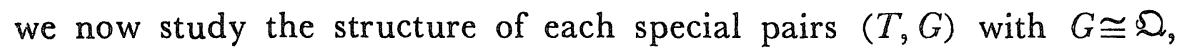
$\mathfrak{I}$ or $\mathfrak{D}$ in some more detail. Recall that a singular abelian surface is an abelian surface $T$ with Picard number $\rho(T)=4$ (corresponding to Case 1, No, 3, of Table 14). In this case $T$ is isogenous to a product $E \times E$ of copies of an elliptic curve $E$ with complex multiplication, and vice versa.

Lemma 5.6. Let $(T, G)$ be a special pair with $G \cong \mathfrak{Q}$, $\mathfrak{I}$ or $\mathfrak{D}$. Then either $a(T)=0$ and $\rho(T)=3$, or $T$ is a singular abelian surface.

Proof. By Lemma 3.4 $\operatorname{End}_{0} T$ contains a definite quaternion algebra. 
According to Table 14 this is possible only in either of the two cases of the lemma.

Let $\mathbb{S}$ be either of $\mathfrak{Q}, \mathfrak{I}$ and $\mathfrak{D}$. We consider the universal family $\left\{\left(T_{q}, G_{q}\right)\right\}_{q \in X}$ of special pairs with $G_{q} \cong \mathbb{S}$ defined in Sect. 3 (cf. (17)) parametrized by $X=\left\{q \in \boldsymbol{H} ; q^{2}=-1\right\} \cong \boldsymbol{P}^{1}$. (Recall that by Theorem 3.11 any maximal special pair is isomorphic to $\left(T_{q}, G_{q}\right)$ for some $q \in X$.) By Lemma 5.6 either $T_{q}$ is a singular abelian surface or $a\left(T_{q}\right)=0$. Then the points $q$ corresponding to singular abelian surfaces $T_{q}$ are identified in the next proposition. For a singular abelian surface $T$ we shall denote by $K_{T}$ the center of the rational endomorphism ring $\operatorname{End}_{0} T$ of $T$.

Proposition 5.7. In the above notation $T=T_{q}$ is a singular abelian surface if and only if there exists a real number $\mu \neq 0$ such that $\mu q \in \Lambda_{\circledast} \subseteq \boldsymbol{H}$. Moreover, in this case we have $K_{T} \cong \boldsymbol{Q}(\mu \sqrt{-1})$. In particular the set $A:=\left\{q \in X ; a\left(T_{q}\right)=2\right\}$ is countable and dense in $X$.

Proof. Let $H=\Lambda_{\Theta} \otimes Q$, which is a definite quaternion algebra over Q. Then $\operatorname{End}_{0} T$ is naturally identified with the ring of endomorphisms $f$ of $H$ as a $\boldsymbol{Q}$-vector space such that $f_{\boldsymbol{R}}$ is $J(q)$-linear, where $J(q)$ is the right multiplication by $q$ on $H_{R} \cong \mathbb{H}$. By left multiplication on $H$ we have the natural embedding $H \longrightarrow \operatorname{End}_{0} T_{q}$. On the other hand, if $\mu q \in \Lambda_{\mathbb{B}}$ for some real number $\mu \neq 0$, then $(\mu q)^{2}=-\mu^{2}$ is a negative rational number. Hence $K:=\{\alpha+\beta \mu q ; \alpha, \beta \in Q\}$ is an imaginary quadratic field. Moreover, by right multiplication $\alpha+\beta \mu q$ (which commutes with $J(q)$ ) is considered as an element of $\operatorname{End}_{0} T_{q}$; hence we have also an embedding $K \longleftrightarrow \operatorname{End}_{0} T_{q}$. Thus we have $\operatorname{End}_{0} T_{q} \supseteqq$ $K \otimes_{Q} H \cong M_{2}(K)$. (Note that by left multiplication $K$ is embeddable also in $H$ so that $K$ is a splitting field of $H$.) As Table 14 shows, $T$ must be a singular abelian surface in this case, and we clearly have $K_{T} \cong K \cong \boldsymbol{Q}(\mu \sqrt{-1})$. Since any $\boldsymbol{Q}$-linear automorphism of $H$ is obtained as the composition of the right and left multiplications of elements of $H$, if there exists no real number $\mu$ as above, $\operatorname{End}_{0} T$ must coincide with $H$. Hence, by Table 14 we see that $a(T)=0$. This shows the proposition. 
5. 4. Next we proceed to describe the rational Neron-Severi group $N_{T, Q}$ of $T=T_{q}$ for each $q \in X$. We start with the description of $H^{2}(T, Q)$ as an inner product space with inner product defined by the cup product. Of course this depends only on the underlying real torus $\mathbb{T}$ of $T$ with the orientation induced by the complex structure of $T$. Recall that we may naturally write $T=\mathbb{H} / \Lambda$, where $\Lambda$ is some lattice in a definite quaternion algebra $H$ over $\mathbb{Q}$ (actually $H=F$ or $F^{\prime}$ (cf. 2.3, (4))), and we have identified $H_{R}$ with $\mathbb{H}$ by a fixed isomorphism $\hat{\varphi}: H_{R} \cong \mathbb{H}$. Here we assume that for the orientation of $\mathbb{H}$ inducing that of $\mathbb{T}, 1, i, j, k$ form a positive basis.

Let $\operatorname{tr}: H \rightarrow Q$ be the (reduced) trace. Define as usual a nondegenerate positive symmetric $Q$-bilinear form $\langle$, $\rangle$ on $H$ by

$$
\langle x, y\rangle=\operatorname{tr}(x \bar{y}), x, y \in H,
$$

where $\bar{y}$ is the quaternion conjugate of $y$. Let $H_{0}$ (resp. $\mathbb{H}_{0}$ ) be the subspace of $H$ (resp. $\mathbb{H}$ ) of pure quaternions, i. e., those quaternions with zero trace. We denote by $H_{0}(-1)$ the space $H_{0}$ equipped with the negative definite inner product $-\langle$,$\rangle . Define a positive integer$ $d=d(\Lambda)$ by

$$
d=\left|\operatorname{det}\left(\left\langle u_{i}, u_{j}\right\rangle\right)\right|
$$

for any $\mathbb{Z}$-basis $u_{i}, 1 \leqq i \leqq 4$, of $\Lambda$. We may identify $H^{2}(T, Q)$ with the space $\wedge^{2} H^{*}$ of $Q$-valued alternating bilinear forms on $\Lambda_{Q} \cong H$, where $H^{*}$ is the dual $Q$-vector space of $H$. Then the multiplicative group $H^{\times}$of $H$ acts naturally on $\wedge^{2} H^{*}=H^{2}(T, Q)$ and the formula

$$
f((\xi, \eta))(x, y)=(1 / \sqrt{d})(\operatorname{tr}(x \bar{\xi} \bar{y})+\operatorname{tr}(x \bar{y} \eta)), \xi, \eta, x, y \in H_{0}
$$

defines a $Q$-linear map $f: H_{0} \oplus H_{0} \rightarrow H^{2}(T, \mathbb{Q})$. Then the follwing is true.

Lemma 5.8. 1) $f$ gives an isomorphism of the inner product spaces $H_{0} \oplus H_{0}(-1)$ and $H^{2}(T, Q)$. 2) If we let $\mathrm{Sp}(1)$ act on $H_{0} \oplus H_{0}(-1)$ by the formula $\sigma(\xi, \eta)=\left(\xi, \sigma \eta \sigma^{-1}\right), \sigma \in \operatorname{Sp}(1), \xi, \eta \in H_{0}$, then $f$ is $\operatorname{Sp}(1)-$ equivariant.

Proof. 1) Denote by $d \mathrm{l}, d i, d j, d k\left(\in H_{R}^{*}\right)$ be the dual basis of $1, i, j, k \in \mathbb{H}=H_{R}$. We denote by the same letter tr the $\mathbb{R}$-linear extension $H_{\boldsymbol{R}} \rightarrow \mathbb{R}$ of $\operatorname{tr}: H \rightarrow \boldsymbol{Q}$. For any $\zeta=a i+b j+c k, a, b, c \in \mathbb{R}$ and $x, y \in H_{R}$ let $\psi_{\zeta}(x, y)=(1 / 2) \operatorname{tr}(x \zeta \bar{y})$ and $\varphi_{\zeta}(x, y)=(1 / 2) \operatorname{tr}(x \bar{y} \zeta)$, con- 
sidered as elements of $\wedge^{2} H_{R}^{*}=H^{2}(T, \boldsymbol{R})$. Then in $\wedge^{2} H_{R}^{*}$ we have

$$
\begin{aligned}
& \phi_{\zeta}=a d 1_{\wedge} d i+b d 1_{\wedge} d j+c d 1_{\wedge} d k-a d j_{\wedge} d k-b d i_{\wedge} d k-c d i_{\wedge} d j \\
& \varphi_{\zeta}=a d 1_{\wedge} d i+b d 1_{\wedge} d i+c d 1_{\wedge} d k+a d j_{\wedge} d k-b d i_{\wedge} d k+c d i_{\wedge} d j .
\end{aligned}
$$

From this we see that for $\xi=a i+b j+c k, \eta=a^{\prime} i+b^{\prime} j+c^{\prime} k \in H_{0, R}$

$$
\phi_{\xi \wedge} \varphi_{\eta}=a a^{\prime}-b b^{\prime}+c c^{\prime}-a^{\prime} a+b^{\prime} b-c^{\prime} c=0
$$

and

$$
\begin{aligned}
& \psi_{\xi \wedge} \psi_{\eta}=2\left(a a^{\prime}+b b^{\prime}+c c^{\prime}\right) \Omega=\langle\xi, \eta\rangle \Omega \\
& \varphi_{\xi \wedge} \varphi_{\eta}=(-2)\left(a a^{\prime}+b b^{\prime}+c c^{\prime}\right) \Omega=-\langle\xi, \eta\rangle \Omega,
\end{aligned}
$$

where $\Omega=d 1_{\wedge} d i_{\wedge} d j_{\wedge}(-d k)=-d 1_{\wedge} d i_{\wedge} d j_{\wedge} d k$. (24) implies that $f\left(H_{0}\right)$ $\perp f\left(H_{0}(-1)\right)$ in $\Lambda^{2} H^{*}$. Hence it suffices to show that $f_{1}:=f_{\mid H_{0}}$ and $f_{2}:=f_{1\left(-H_{0}\right)}$ are both inner product preserving; indeed, then the injectivity of $f$ follows immediately and therefore $f$ is isomorphic since both $H_{0} \oplus H_{0}$ and $H^{2}(T, \boldsymbol{Q})$ are 6-dimensional.

Let $u_{i}, 1 \leqq i \leqq 4$, be an oriented basis of $\Lambda$. Then if we set $\Omega_{\Lambda}=$ $d u_{1 \wedge} d u_{2 \wedge} d u_{3 \wedge} d u_{4}$, we have $\Omega=(d / 4) \Omega_{\Lambda}$. From (25) and this we get $\psi_{\xi \wedge} \psi_{\eta}=\langle\xi, \eta\rangle(d / 4) \Omega_{\Lambda}$; so $f_{1}(\xi)_{\wedge} f_{1}(\eta)=\left(2 \psi_{\xi} / \sqrt{d}\right)_{\wedge}\left(2 \psi_{\eta} / \sqrt{d}\right)=(4 / d) \psi_{\xi \wedge} \psi_{\eta}=$ $\langle\xi, \eta\rangle \Omega_{\Lambda}$. This shows that $f_{1}$ is inner product preserving. Similarly, we can prove the same result also for $f_{2}$. 1) is thus proved. 2) can also be checked by direct computation as above.

Now, returning to our complex torus $T=T_{q}, q \in X$, let $H^{p, q}, p+q=2$, be the Hodge $(p, q)$-components of $H^{2}(T, C)$. The action of $H^{\times}$ on $H^{2}(T, \boldsymbol{Q})$ extends naturally to a complex linear action on $H^{2}(T, C)$, and preserves the Hodge components $H^{p, q}$, and hence also $N_{T, \boldsymbol{Q}}=H^{1,1} \cap H^{2}(T, \boldsymbol{Q})$ and $B_{T, \boldsymbol{Q}}=N_{T, \boldsymbol{Q}}^{\perp}$ in $H^{2}(T, \boldsymbol{Q})$. (Note that $H \cong \operatorname{End}_{0} T$ and $\mathrm{Sp}(1)$ acts on $H^{2}(T, Q)$ isometrically.) Now we shall identify $H^{2}(T, \boldsymbol{C})$ with $H_{0, c} \oplus H_{0}(-1)_{c}$ via $f_{c}^{-1}$. Accordingly, $N_{T, Q}$ and $B_{T, Q}$ are considered naturally as subspaces of the latter. Further, via $\hat{\varphi}_{0}: H_{0, R} \cong \boldsymbol{H}_{0}$ we shall consider $q \in X \subseteq \boldsymbol{H}_{0}$ as an element of $H_{0, R} \cong H_{0, c}$.

Proposition 5.9. With respect to the above identifications we have $H^{1,1}=\boldsymbol{C} q \oplus H_{0}(-1)_{c}$. Moreover, $a(T)=2 \quad(r e s p .=0)$ if and only if there exists (resp. does not exist) a real number $\mu \neq 0$ such that $\mu q \in H_{0}$; in this case we have $N_{T, \boldsymbol{Q}}=\boldsymbol{Q}(\mu q) \oplus H_{0}(-1), B_{T, \boldsymbol{Q}}=\boldsymbol{Q}(\mu q)^{\perp}$ in $H_{0}$ and $N_{T, \boldsymbol{Q}}^{H^{\times}}=$ $\boldsymbol{Q}(\mu q) \quad\left(\right.$ resp. $N_{T, \boldsymbol{Q}}=H_{0}(-1), B_{T, \boldsymbol{Q}}=H_{0}$ and $\left.N_{T, \boldsymbol{Q}}^{H^{\times}}=0\right)$. 
Proof. We see that for any $\xi, \eta \in H_{0, R}$ and $x, y \in H_{\mathbb{R}}$,

$$
\sqrt{d} f_{R}((\xi, \eta))(x q, y q)=\operatorname{tr}(x q \overline{\xi y q})+\operatorname{tr}(x q \overline{y q} \eta)=\operatorname{tr}\left(x q \xi q^{-1} \bar{y}\right)+\operatorname{tr}(x \bar{y} \eta)
$$

since $q \bar{q}=1$. It follows that for fixed $\xi, \eta$ we have

$$
f_{R}((\xi, \eta))(x q, y q)=f_{R}((\xi, \eta))(x, y), x, y \in H_{R}
$$

(which is equivalent to the condition that $f((\xi, \eta)) \in H_{R}^{1,1}:=H^{1,1} \cap$ $H^{2}(T, R)$ ), if and only if $q$ commutes with $\xi$, i. e., $\nu q=\xi$ for some real number $\nu_{0}$ Hence we have $H_{\mathbb{R}}^{1,1}=\mathbb{R} q \oplus H_{0}(-1)_{\mathbb{R}}$. From this the first assertion follows immediately. This then in turn implies that $\rho(T)=4$ (resp. 3) if and only if there exists (resp. does not exist) a real number $\mu$ such that $\mu q \in H_{0}$. This is equivalent to the second assertion by Theorem 5.1. Further, in this case we clearly get the desired description of $N_{T, Q}$ and $B_{T, Q}$. The description of $N_{T, Q}^{H^{\times}}$as in the proposition follows from this and 2) of the previous lemma.

Remark 5. 10. Suppose that $N_{T, Q}=\mathbb{Q}(\mu q) \oplus H_{0}(-1)$, then we may assume that $\mu q \in N_{T}$ by replacing $\mu$ by $n \mu$ for a large positive integer $n$. Let $L$ be a line bundle on $T$ whose chern class is $\mu q$. Then since $\langle\mu q, \mu q\rangle>0$, either $L$ or $L^{-1}$ is ample.

Since $G$ generates $H$ as a -algebra, from Proposition 5.9 and Remark 5.10 we obtain the following:

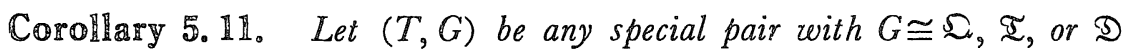
and with $T$ a singular abelian surface. Then the $G$-invariant Neron-Severi lattice $N_{T}^{G}$ is of rank 1 , and it contains a chern class of an ample line bundle.

By the above corollary $N_{T}^{G}$ is a unary positive even lattice (cf.6.1), and has a unique positive generator, say $e$. Then we define a positive integer $\delta$ by

$$
\delta=\langle e, e\rangle / 2
$$

and call it the degree of $(T, G)$. For instance $G$ leaves invariant a principal polarization on $T$ if and only if $\delta=1$.

Remark 5.12. In the course of the proof of Proposition 5.9 we have seen that $\left(H_{R}^{1,1}\right)^{H^{\times}}=\mathbb{R} q$. Hence $\omega_{q}:=\operatorname{tr}(x q \bar{y})$ is the unique $H^{\times}-$ 
invariant real $(1,1)$-form on $T_{q}$ up to constants. On the other hand, the form $\omega_{q}$ can be interpreted as follows. Let $g(x, y)=\operatorname{tr}(x \bar{y})$. Then $g$ is a non-degenerate symmetric bilinear form on $H_{R} \cong \mathbb{H}$. For any $q, g_{q}(x, y):=g(x, y)+\sqrt{-1} g(x q, y)$ defines a hermitian metric on the complex vector space $(\boldsymbol{H}, q)$. Hence it defines a Kähler metric on $T$ (denoted still by $g_{q}$ ) whose underlying Riemannian metric is independent of the choice of $q$ (cf. Calabi [3]). Thus $\omega_{q}$ is nothing but the Kähler form associated to $g_{q}$. Further, for a positive real number $\mu, \mu g_{q}$ is a (rational) Hodge metric in the sense that $\mu \omega_{q}$ belongs to $H_{Q}^{1,1}$ if and only if $\mu q \in H_{0}$. Note further that $H_{0}(-1)$ is just the subspace of $H_{\dot{Q}}^{1}{ }^{1}$ consisting of those classes which are primitive with respect to the Kähler metric $g_{q}$ and is independent of the choice of $q$ (cf. [3, Lemma 4.2]).

Under a suitable formulation Proposition 5.9 and Remark 5.10 are true for any 2-dimensional complex torus $T$ which admits a definite quaternion algebra over $\mathbb{Q}$ as a subalgebra of $\operatorname{End}_{0} T$.

5. 5. For a singular abelian surface $T$ we denote by $m_{T}$ the unique square-free positive integer $m$ such that $K_{T} \cong Q(\sqrt{-m})$, where $K_{T}$ is the center of $\operatorname{End}_{0} T$. We end this section by proving the following:

Proposition 5.13。Let $\mathfrak{B S}=\mathfrak{Q}$ or $\mathfrak{I}$ (resp. D). Let $m$ be a squarefree positive integer. Then there exists a special pair $(T, G)$ such that $G \cong \mathbb{S}$ and $T$ is a singualr abelian surface with $m_{T}=m$ if and only if $m \neq \equiv-1$ (8) (resp. $\equiv-1$ (3)).

Proof. We consider only the case where $\mathfrak{S}=\mathfrak{Q}$ or $\mathfrak{I}$, the other cases being similar. Let $T$ be a singular abelian surface with $K_{T}=Q(\sqrt{-m})$. First, we note the following implications; " $T$ admits a special action of $(6) " \rightarrow$ " $H$ is embedded in $M_{2}\left(K_{T}\right) \cong \operatorname{End}_{0} T$ " $\leftrightarrow$ " $K_{T}$ is a splitting field of $H$ " $\leftrightarrow$ " $K_{T}$ is embedded in $H$ " $" \xi^{2}=-m$ for some $\xi \in H " \leftrightarrow$ " $a^{2}+b^{2}+c^{2}=m$ for some rational numbers $a, b, c " \leftrightarrow$ " $m \not \equiv-1$ (8)" (cf. [18, p45, Lemma A]). This shows already the necessity and shows also that if $m \not \equiv-1$ (8), $\& 5$ is embedded in $\operatorname{SL}\left(2, K_{T}\right)$. In this case let $\Lambda$ be any maximal $\mathfrak{0}$-order of $H$, where $\mathfrak{o}$ is the maximal order of $K_{T}$. Then $\Lambda$ is of the form $\Lambda=\operatorname{Hom}_{\mathfrak{o}}(M, M)$ for an 0 -module 
$M$ of rank 2; further $M$ is up to isomorphism of the form $\mathfrak{a} \oplus \mathfrak{\Im}$ for some ideal $\mathfrak{S}$ of $\mathfrak{o}$ (cf. $[17,4.13]$ ). Fix an embedding $K \longrightarrow \mathbb{C}$; then $T:=E_{1} \times E_{2}, E_{1}=\mathbb{C} / \mathfrak{o}, E_{2}=\mathbb{C} / \mathfrak{S}$, admits a natural special action of $\mathscr{S}$.

We note that Proposition 5.13 can also be deduced from Theorem 7.2 and 7.5 below, though the above proof is more direct.

\section{§6. Special Pairs and Root Lattices}

In Sect. 6 we associate to each group \&S in Lemma 3.3 a certain root lattice, and by using this we shall give a characterization of those complex tori $T$ which admits a special action of \&S in terms of the Neron-Severi and transcendental lattices. The conditions can be made more explicit in the case of singular abelian surfaces; indeed in Sect. 7 we shall determine all the singular abelian surfaces which admit special \&s-actions in terms of their transcendental lattices in case (s) is non-commutative. Moreover, in this case we also determine the number of conjugacy classes of such special subgroups in Aut $T$.

6. 1. We recall some basic terminologies on Euclidian lattices used in this section. Let $L$ be a lattice. Then for any rational number $m$ we shall denote by $L(m)$ the lattice $L$ with the new inner product $\langle,\rangle_{n}$ defined by $\langle x, y\rangle_{m}=m\langle x, y\rangle$ (as long as the right hand side is always an integer). Let $e_{i}, 1 \leqq i \leqq n$, be a basis of $L$. Then the discriminant of $L$ is the determinant of the matrix $\left(\left\langle e_{i}, e_{j}\right\rangle\right)_{1 \leqq i, j \leqq n}$. $L$ is said to be even if $\langle x, x\rangle$ is even for any $x \in L$. An orieniation of $L$ is by definition an orientation of the real vector space $L_{R}$. We denote by $\mathrm{O}(L)$ and $\mathrm{SO}(L)$ the orthogonal and special orthogonal groups of $L$ respectively. If $K$ is a sublattice of $L, K^{\perp}$ denotes its orthogonal complement in $L . L_{1} \perp L_{2}$ denotes an orthogonal direct sum. $U$ will denote the standard hyperbolic lattice; $U=\mathbb{Z} e_{1} \oplus \mathbb{Z} e_{2}$ with $\left\langle e_{i}, e_{i}\right\rangle=0$ and $\left\langle e_{1}, e_{2}\right\rangle=1$. If $\mathrm{T}$ is a complex torus of dimension 2 , then $H^{2}(T, \mathbb{Z})$ is isomorphic as a lattice to $U^{3}=U \perp U \perp U$. A sublattice $K$ of $L$ is said to be primitive if the quotient $L / K$ has no torsion. An embedding $j: K \longleftrightarrow L$ is said to be primitive if $j(K)$ is primitive. Two sublattices $K_{\alpha}$ (resp. two embeddings $j_{\alpha}: K \longrightarrow L$ ), $\alpha=1,2$, are said to be equivalent if there exists an element $\xi \in \mathrm{O}(L)$ such that $\xi\left(K_{1}\right)=K_{2}\left(\right.$ resp. $\left.\xi j_{1}=j_{2}\right)$. 
Let $A$ be a finite abelian group. Then a quadratic form on $A$ is by definition a map $q: A \rightarrow Q / 2 \mathbb{Z}$ such that 1) for any $x \in A$ and for any integer $\alpha$ we have $q(\alpha x)=\alpha^{2} q(x)$ and 2) there exists a $Q / Z$ valued bilinear form $b$ on $A$ satisfying $q(x+y)-q(x)-q(y)=2 b(x, y)$ for any $x, y \in A$; here $b$ is uniquely determined by $q$. We call the pair $(A, q)$ a quadratic form group. We say that $q$ is non-degenerate if so is $b$.

Let $L$ be an even lattice. Let $L^{*}$ be the dual lattice of $L ; L^{*}=$ $\left\{x \in L_{Q} ;\langle x, y\rangle_{Q} \in \mathbb{Z}\right.$ for any $\left.y \in L\right\}$. Then the formula $q(x+L)=$ $\langle x, x\rangle \bmod 2 \mathbb{Z}\left(x \in L^{*}\right)$ defines a nondegnerate quadratic form $q_{L}$ on the finite abelian group $A:=L^{*} / L$. This $q_{L}$, or rather, the associated quadratic form group $\left(A, q_{L}\right)$, is called the discriminant form (group) of $L$. We have

$$
|A|=\text { discriminant of } L \text {, }
$$

where $|A|$ is the order of $A$. We denote the automorphism group of $(A, q)$ by $\mathrm{O}(A, q)$, or simply by $\mathrm{O}(q)$.

6. 2. In this subsection we shall give a description of the moduli space of 2-dimensional complex tori in terms of 2-forms, according to Shioda [22]. Let $\Lambda^{0}=\mathbb{Z}^{4}$ and $\Lambda_{0}$ its dual. Fix an orientation on $\Lambda^{0}$ and hence on $\Lambda_{0}$. Then we have the natural pairing $\wedge^{2} \Lambda_{0} \times \wedge^{2} \Lambda_{0} \rightarrow$ $\wedge^{4} \Lambda_{0} \cong Z^{4}$ which makes $\wedge^{2} \Lambda_{0}$ a euclidian lattice isomorphic to $U^{3}$ 。

Let $T$ be a complex torus of dimension 2. Write $T=E / \Lambda$. Then via the natural real linear isomorphism $\lambda_{R}: \Lambda_{R} \rightarrow E, \Lambda=H_{1}(T, \mathbb{Z})$ is given a natural orientation. Then a marking $\varphi: \Lambda \rightarrow \Lambda^{0}$ is said to be admissible if it is orientation preserving. $A$ 2-marking of $T$ is by definition an isomorphism $\psi: H^{2}(T, \mathbb{Z}) \rightarrow U^{3}$ of euclidian lattices. Any (1-) marking $\varphi$ as above induces the 2-marking $\wedge(\varphi): H^{2}(T, \mathbb{Z}) \rightarrow U^{3}$ 。 Then a 2-marking $\psi$ is said to be admissible if there exists an admissible marking $\varphi$ of $T$ such that $\psi=\wedge(\varphi)$. We define isomorphisms of two admissibly 2-marked complex tori in the obvious way.

Let $H^{2,0}(T)$ be the Hodge $(2,0)$-component of $H^{2}(T, C)$. Let

$$
E(T)=H^{2}(T, R) \cap\left(H^{2,0}(T) \oplus \bar{H}^{2,0}(T)\right),
$$

where - denotes the complex conjugation. The projection induces the real linear isomorphism $E(T) \cong H^{2,0}(T)$, which induces the natural orientation on $E(T)$. With this orientation we consider $E(T)$ as an 
oriented 2-plane in $H^{2}(T, \mathbb{R})$. We note that we have the inclusion $E(T) \longrightarrow B_{T, R}$. Let $(T, \phi)$ be a 2 -marked complex torus. Then we set

$$
E(T, \psi):=\psi(E(T)) \longrightarrow U_{R^{\circ}}^{3}
$$

With the orientation induced from $E(T)$ we consider $E(T, \phi)$ as an oriented 2-plane in $U_{R}^{3}$, and call it the period of $(T, \psi)$.

Let $V$ be the set of oriented 2-planes $E$ in $U_{R}^{3}$. Then $V$ has the natural structure of a connected homogeneous complex manifold

$$
V \cong \mathrm{SO}_{0}(3,3) / \mathrm{SO}(2) \times \mathrm{SO}_{0}(1,3)
$$

with respect to some identification

$$
\mathrm{O}(3,3)=\mathrm{O}\left(U_{R}^{3}\right) \text {. }
$$

Here $\mathrm{SO}_{0}(p, q)$ is the identity component of $O(p, q)$. Moreover the correspondence $(T, \psi) \rightarrow E(T, \psi) \in V$ induces the natural bijective correspondence between the set of isomorphism classes of admissibly 2-marked complex tori and the set $V$ (cf. [22]).

Any admissibly (1-) marked complex torus $(T, \varphi)$ naturally gives rise to an admissibly 2 -marked complex torus $(T, \psi)$ by setting $\psi=\wedge(\varphi)$. This induces an isomorphism of homogeneous complex manifolds

$$
\delta: W^{+} \cong V
$$

if $W^{+} \cong \mathrm{SL}_{4}(\mathbb{R}) / \mathrm{GL}_{2}(\mathbb{C})$ is considered naturally as the (fine) moduli space of admissibly marked complex tori. Indeed, with respect to the homogeneous structures as above $\delta$ is compatible with the natural double covering map

$$
\delta_{0}: \mathrm{SL}_{4}(\mathbb{R}) \rightarrow \mathrm{SO}_{0}\left(U_{R}^{3}\right)
$$

of real Lie groups which induces the surjection $\mathrm{SL}_{4}(\mathbb{Z}) \rightarrow \mathrm{SO}_{0}\left(U^{3}\right)$. Here, by definition,

$$
\mathrm{SO}_{0}\left(U^{3}\right)=\mathrm{O}\left(U^{3}\right) \cap \mathrm{SO}_{0}\left(U_{R}^{3}\right),
$$

with $\mathrm{SO}_{0}\left(U_{R}^{3}\right)$ the identity component of $\mathrm{O}\left(U_{R}^{3}\right) \cong \mathrm{O}(3,3)$. In particular $\mathrm{SO}_{0}\left(U^{3}\right) \backslash V$ may also be considered as the moduli space of 2dimensional complex tori.

Let $M$ be any primitive sublattice of $U^{3}$. Let

$$
V_{M}=\left\{E \in V ; E \subseteq M_{R}\right\} 。
$$

Then, if $M$ is nondegenerate with signature $(s, t), s \geqq 2, V_{M}$ is naturally a homogeneous complex submanifold of $V$; namely 


$$
V_{M} \cong \mathrm{SO}(s, t) / \mathrm{SO}(2) \times \mathrm{SO}(s-2, t) .
$$

We note that if $s=3$, we have the inclusion

$$
\mathbb{P}^{1} \cong \mathrm{SO}(3) / \mathrm{SO}(2) \longrightarrow \mathrm{SO}(s, t) / \mathrm{SO}(2) \times \mathrm{SO}(s-2, t) \cong V_{M} .
$$

6. 3. For any lattice with discriminant form $(A, q)$ we denote by $H(L)$ the kernel of the natural homomorphism $\mathrm{O}(L) \rightarrow \mathrm{O}(q)$. Then we set

$$
S H(L)=H(L) \cap \mathrm{SO}(L) .
$$

The following lemma is due to Nikulin.

Lemma 6.1. 1) Let $L$ be an even lattice of rank $k$. Then there exists a natural embedding $L \perp L(-1) \longrightarrow U^{k}$ such that $L$ and $L(-1)$ are primitive in $U^{k}$ and that any element $g \in \mathrm{O}(L)$ extends naturally to an element $\tilde{g}$ of $\mathrm{SO}\left(U^{k}\right)$. 2) Let $L$ be any primitive sublattice of $U^{k}$. Then any element $g \in S H(L)$ is a restriction of a unique element $\tilde{g}$ of $\mathrm{SO}\left(U^{k}\right)$ such that the restriction $\left.\tilde{g}\right|_{L^{\perp}}$ of $\tilde{g}$ to $L^{\perp}$ is the identity.

Proof. See Nikulin $[15,1.6 .3,1.1 .1]$ and $[14$, Prop. 1.1].

For any pair $(T, G)$ we shall denote by $H^{2}(T, \mathbb{Z})^{G}$ the primitive sublattice of $H^{2}(T, \mathbb{Z})$ of $G$-fixed elements, and by $H^{2}(T, \mathbb{Z})_{G}$ its orthogonal complement in $H^{2}(T, \mathbb{Z})$. The following lemma is due also to Nikulin [14].

Lemma 6.2. Let $(T, G)$ be a special pair. Then the following hold: 1) We have the inclusions $B_{T} \longrightarrow H^{2}(T, \mathbb{Z})^{G}$ and $H^{2}(T, \mathbb{Z})_{G} \longrightarrow N_{T}$, 2) $H^{2}(T, \mathbb{Z})_{G}$ is negative definite and 3 ) the natural image of $G$ in $\mathrm{O}\left(H^{2}(T, \mathbb{Z})_{G}\right)$ is contained in $\operatorname{SH}\left(H^{2}(T, \mathbb{Z})_{G}\right)$.

Proof. 1) follows from the definition of special action and [14, Th. 3. 1, a)]. 2) follows from [14, Th. 4.3, a)], which also holds for general complex tori. Finally, 3) follows from 1).

Let $(L, H, E)$ be a triple consisting of a) a negative primitive sublattice $L$ of $U^{3}$, b) a subgroup $H$ of $S H(L)$ with the set of $H$ invariants $L^{H}=\{0\}$, and c) an oriented 2-plane $E$ in $L_{\vec{R}}^{\perp}$. (Here the 
case $L=\{0\}$ is allowed in a).) Let $\mathscr{B}$ be the set of such triples. Let $(T, G)$ be any special pair with $-1 \in G$. Let $\psi: H^{2}(T, \mathbb{Z}) \rightarrow U^{3}$ be an admissible 2-marking. Set $L=\psi\left(H^{2}(T, \mathbb{Z})_{G}\right), H=$ the image. $\bar{G}$ of $G$ in $\mathrm{O}(L) \cong \mathrm{O}\left(H^{2}(T, \mathbb{Z})_{G}\right)$ and $E=E(T, \phi)$ with its natural orientation. Then by Lemma $6.2 L$ is negative and $H \cong S H(L)$ with $L^{H}=\{0\}$ 。 Thus the triple $(L, H, E)$ is in $\mathscr{B}$.

Let $\mathscr{S}$ be the set of isomorphism classes of admissibly 2-marked special pairs $(T, G ; \phi)$ with $-1 \in G$ (with isomorphisms defined in the obvious way). We have then obtained the natural mapping $\beta: \mathscr{S} \rightarrow \mathscr{B}$ defined by

$$
(T, G ; \psi) \rightarrow\left(\psi\left(H^{2}(T, \mathbb{Z})_{G}\right), \vec{G}, E(T, \psi)\right) .
$$

We say that two triples $(L, H, E)$ and $\left(L^{\prime}, H^{\prime}, E^{\prime}\right)$ in $\mathscr{B}$ are equivalent if there exists an element $u \in \mathrm{SO}_{0}\left(U^{3}\right)$ such that $u(L)=L^{\prime}, u_{*}(H):=$ $u H u^{-1}=H^{\prime}$ and that $u_{R}(E)=E^{\prime}$ with the resulting isomorphism $E \rightarrow E^{\prime}$ orientation preserving. Let $\overline{\mathscr{B}}$ be the set of equivalence classes of triples in $\mathscr{B}$. Then we see that $\beta$ induces the natural map $\bar{\beta}: \overline{\mathscr{S}} \rightarrow \overline{\mathscr{B}}$, where $\overline{\mathscr{S}}$ is the set of isomorphism classes of special pairs $(T, G)$ with $-1 \in G$.

Proposition 6. 3. The naiural maps $\beta: \mathscr{B} \rightarrow \mathscr{S}$ and $\bar{\beta}: \overline{\mathscr{B}} \rightarrow \overline{\mathscr{S}}$ defined above are bijective.

Proof. Injectivity. Let $i=1,2$. Let $\left(L_{i}, H_{i}, E_{i}\right)$ be the triples coming from admissibly 2-marked special pairs $\left(T_{i}, G_{i} ; \psi_{i}\right)$ with $-1 \in G_{i \circ}$ Suppose that they are equivalent with an equivalence $u \in \mathrm{SO}_{0}\left(U^{3}\right)$ as above. Then by the definition of $\beta$, the induced isomorphism $v:=\psi_{2}^{-1} u \psi_{1}: H^{2}\left(T_{1}, \mathbb{Z}\right) \rightarrow H^{2}\left(T_{2}, \mathbb{Z}\right)$ has the property that it is induced by an orientation preserving isomorphism $H_{1}\left(T_{1}, \mathbb{Z}\right) \rightarrow$ $H_{1}\left(T_{2}, \mathbb{Z}\right), \quad v\left(H^{2}\left(T_{1}, \mathbb{Z}\right)^{G_{1}}\right)=H^{2}\left(T_{2}, \mathbb{Z}\right)^{G_{2}}, \quad v\left(H^{2}\left(T_{1}, \mathbb{Z}\right)_{G_{1}}\right)=H^{2}\left(T_{2}, \mathbb{Z}\right)_{G_{2}}$, $v_{R}\left(E\left(T_{1}\right)\right)=E\left(T_{2}\right)$ with $E\left(T_{1}\right) \rightarrow E\left(T_{2}\right)$ orientation preserving, and that $v_{*}\left(\bar{G}_{1}\right)=\bar{G}_{2}$, where $\bar{G}_{i}$ is the natural image of $G_{i}$ in $O\left(\mathbb{H}^{2}(T, \mathbb{Z})_{G_{i}}\right)$. Therefore if $\bar{G}_{i}^{\prime}$ is the image of $G_{i}$ in $O\left(H^{2}(T, \mathbb{Z})\right)$, then we also have $v_{*}\left(\bar{G}_{1}^{\prime}\right)=\bar{G}_{2}^{\prime}$. Then by Shioda [22] (cf. (6.2)) $v$ is induced by an isomorphism $f: T_{1} \rightarrow T_{2}$ of complex tori. Further from the relations $v_{*}\left(\bar{G}_{1}^{\prime}\right)=\bar{G}_{2}^{\prime}$ noted above and $G_{i} /\langle-1\rangle \cong \bar{G}_{i}^{\prime}$, we have $f_{*}\left(G_{1}\right)=G_{2}$, i。 e e, $\left(T_{1}, G_{1}\right) \cong\left(T_{2}, G_{2}\right)$. This shows the injectivity of $\bar{\beta}_{\text {. If }}\left(L_{1}, H_{1}, E_{1}\right)=$ 
$\left(L_{2}, H_{2}, E_{2}\right.$ ), then we can take $u$ to be the identity in the above argument; hence we have $\phi_{1}=f_{*} \psi_{2}$, where $f_{*}: H^{2}\left(T_{1}, \mathbb{Z}\right) \rightarrow H^{2}\left(T_{2}, \mathbb{Z}\right)$ is induced by $f$. This shows the injectivity of $\beta$.

Surjectivity. It suffices to show the surjectivity of $\beta$. Let $(L, H, E)$ be an arbitrary triple in $\mathscr{B}$. Then by [22] (cf。(6.2)) there exists an admissibly 2-marked complex torus $(T, \phi)$ such that $E(T, \psi)=E$. On the other hand, from the inclusion $H \cong S H(L)$ and Lemma 6.1 any $h \in H$ extends to a unique element $\bar{h} \in \mathrm{SO}\left(U^{3}\right) \operatorname{such}$ that $\left.h\right|_{L^{\perp}}=\mathrm{id}_{L^{\perp}}$ 。 We can thus consider $H$ as a subgroup of $\mathrm{SO}\left(U^{3}\right)$. Set $\tilde{H}=$ $\left\langle H,-\mathrm{id}_{v^{3}}\right\rangle \cong H \times\left\langle-\mathrm{id}_{v^{3}}\right\rangle$, and $H_{0}=\tilde{H} \cap \mathrm{SO}_{0}\left(U^{3}\right)$. Then $H_{0} \cong H$ and again by Shioda [22] any $h_{0} \in H_{0}$ is induced by an automorphism of $T$ determined uniquely modulo multiplication by -1 . It follows that there exists a unique subgroup $G$ of Aut $T$ with $-1 \in G$ such that the natural image of $G$ in $\mathrm{SO}\left(U^{3}\right) \cong \mathrm{SO}\left(H^{2}(T, \mathbb{Z})\right)$ coincides with $H_{0}$. Suppose now that $(T, G)$ is special. Then by Lemma 6.2 we must have $H_{0}=H$; then $(L, H, E)$ is the image of $(T, G ; \psi) \in \mathscr{S}$ by $\beta$, proving the surjectivity of $\beta$.

It remains to show that $(T, G)$ is special. Set $M=L^{\perp}$. Now in the above argument we let $E$ vary in $V_{M}$ with $L$ and $H$ fixed. Let $W_{M}=\delta^{-1}\left(V_{M}\right) \subseteq W^{+}$. Let $H=\delta_{0}^{-1}\left(H_{0}\right)$, where $\delta_{0}$ is defined by (27). Then the above argument shows that any admissibly marked pair $(T ; \varphi)$ corresponding to a point of $W_{M}$ can be completed to a marked pair $\left(T, G_{i}^{\circ} \varphi\right)$ of type $H$ (cf. 2.1, b)). It follows that $W_{M} \cong W_{\hat{H}}$ in the notation of $2.1, b)$. On the other hand, since $L$ is negative, the signature of $M$ is of the form $(3, t)$ with $0 \leqq t \leqq 3$. Therefore $W_{M} \cong V_{M}$ contains at least one projective line $\boldsymbol{P}^{1}$ (cf. (28)). Then by Proposition 2.17 and the finiteness of exceptional pairs (cf. 4.3) $W_{\hat{H}}$ must be isomorphic to one of the following homogeneous complex manifolds $W^{+}, G L_{2}(\boldsymbol{C}) / \mathbb{C}^{* 2}$ and $\mathbb{H}^{\times} / \mathbb{C}^{*}$. But we know already that then any pair corresponding to a point of $W_{\hat{H}}$ is special. Hence $(T, G)$ also is special.

q. e.d.

6. 4. Let $(T, G)$ be a special pair with $-1 \in G$. Let $\phi$ be any admissible 2-marking of $(T, G)$. Let $(L, H, E)$ be the triple corresponding to $(T, G, \psi)$ via $\beta$. Then from the definition of $\beta$ we see that the equivalence class of the pair $(L, H)$ is constant when $(T, G)$ vary on each connected component of the moduli space (cf. Sect. 3 ). 
(Here two $(L, H)$ and $\left(L^{\prime}, H^{\prime}\right)$ are equivalent if and only if there exists an element $u \in \mathrm{O}\left(U^{3}\right)$ such that $u(L)=L^{\prime}$ and $u_{*}(H)=H^{\prime}$.) In particular if $G \nsubseteq \mathscr{Q}$, then the equivalence class of $(L, H)$ is determined uniquely by the abstract isomorphism class of $\mathbb{G}$, and if $G \cong \mathfrak{Q}$, we have two possibilities according as $(T, G)$ is maximal or not. We shall now determine these isomorphism classes of $(L, H)$ explicitly. It turns out that such $L$ are actually given as certain root lattices.

Any root system $R$ defines the associated (negative) root lattice, which we shall again denote by the same letter $R$. For instance $A_{k}$ is the lattice with an integral basis $e_{1}, \ldots, e_{k}$ with $\left\langle e_{i g} e_{i}\right\rangle=-2$, $\left\langle e_{i}, e_{i+1}\right\rangle=1,1 \leqq i \leqq k-1$, and $\left\langle e_{i}, e_{j}\right\rangle=0$ if $|i-j| \geqq 2$. We shall give the structure of automorphism groups of certain root lattices in the following table (cf. $[11,12.2]$ ):

Table 15

\begin{tabular}{|c|c|c|c|c|}
\hline rank & $L$ & $\mathrm{O}(L)$ & $H(L)$ & $S H(L)$ \\
\hline 2 & $\begin{array}{l}A_{1}^{2} \\
A_{2}\end{array}$ & $\begin{array}{l}\mathfrak{S}_{2}^{2} \rtimes \mathfrak{S}_{2} \\
\mathfrak{S}_{3} \rtimes \mathfrak{S}_{2}\end{array}$ & $\begin{array}{l}\mathfrak{S}_{2}^{2} \\
\mathfrak{S}_{3}\end{array}$ & $\begin{array}{l}\mathfrak{\mho}_{2}=\langle-1\rangle \\
\mathfrak{\mho}_{3}\end{array}$ \\
\hline 3 & $\begin{array}{c}A_{1}^{3} \\
A_{3} \\
A_{2} \perp A_{1}\end{array}$ & $\begin{array}{c}\mathfrak{S}_{2}^{3} \times \mathfrak{S}_{3} \\
\mathfrak{S}_{4} \times \backslash \mathfrak{G}_{2} \\
\left(\mathfrak{S}_{3} \times \mathfrak{\Im}_{2}\right) \times \mathfrak{S}_{2}\end{array}$ & $\left|\begin{array}{c}\mathfrak{S}_{2}^{3} \\
\mathfrak{S}_{4} \\
\mathfrak{S}_{3} \times \mathfrak{E}_{2}\end{array}\right|$ & $\begin{array}{l}\mathfrak{\Im}_{2}^{2}=\langle(1,-1,-1),(-1,-1,-1)\rangle \\
\mathfrak{U}_{4} \\
\mathfrak{S}_{3} \cong \Gamma_{\sigma}, \sigma: \mathbb{S}_{3} \rightarrow \mathfrak{C}_{2}\end{array}$ \\
\hline
\end{tabular}

We note that $H(L)$ is just the Weyl group of $L$ in each case and that these root lattices exhaust all the negative even root lattices of rank 2 and 3. (We have $G_{2} \cong A_{2}$ and $C_{3} \cong A_{3}$ as root lattices.)

Let $\mathbb{S}$ be one of the following groups: $\mathfrak{C}_{m}, m=4,6, \mathfrak{Q}, \mathfrak{I}$, or $\mathfrak{D}$. Then we define the negative even root lattice $L_{\circledast}$ as a root lattice as follows:

$$
\begin{aligned}
& L_{\mathbb{\varpi}_{4}}=A_{1}^{2}, \quad L_{\mathbb{\Xi}_{6}}=A_{2} \\
& L_{\mathrm{\curvearrowright}}=A_{1}^{3}, \quad L_{\mathfrak{q}}=A_{3}, \quad L_{\triangleright}=A_{2} \perp A_{1} .
\end{aligned}
$$

From Table 15 we see immediately that

$$
\mathrm{SH}\left(L_{\mathbb{\Theta}}\right) \cong G /\langle-1\rangle \quad \text { and } \quad L_{\mathbb{\Theta}}^{S H\left(L_{\mathbb{\Theta}}\right)}=0 .
$$

We also set 


$$
\begin{aligned}
& M_{\oplus}=L_{\oplus}(-1) \perp U \text { if } \mathbb{S}=\wp_{m} \\
& =L_{\mathbb{B}}(-1) \quad \text { if } \mathbb{S} \neq \mathfrak{C}_{m} \text {. }
\end{aligned}
$$

By Lemma 6.1 we have the natural embedding

$$
j_{\oplus}: M_{\leftrightarrow} \perp L_{\oplus} \longrightarrow U^{3} \text {, }
$$

in which $M_{\oplus}$ and $L_{\circledast}$ are the orthogonal complement of each other. In what follows we consider $M_{\circledast}$ and $L_{\circledast}$ primitive sublattices of $U^{3}$ via $j_{\Theta}$.

Theorem 6.4. Let \&s be as above. Let $(T, G)$ be a special pair with $-1 \in G$ and with $G \cong \mathbb{S}$. Let $\bar{G}$ be the natural image of $G$ in $\mathrm{O}\left(H^{2}(T, \mathbb{Z})_{G}\right)$ so that $\bar{G} \cong G /\langle-1\rangle$. 1) Suppose that $\mathbb{S} \neq \mathfrak{夭}_{2}$ and $(T, G)$ is maximal when $\mathbb{S}=\mathfrak{O}$. Then there exists an admissible 2-marking $\phi$ of $T$ such that $\phi\left(H^{2}(T, Z)^{G}\right)=M_{\mathbb{B}}$ and $\phi\left(H^{2}(T, \mathbb{Z})_{G}\right)=L_{\mathbb{\Theta}}$. Moreover, $\phi$ induces an isomorphism of $\bar{G}$ with $S H\left(L_{\mathbb{G}}\right)$. 2) If $\mathscr{S}=\mathfrak{Q}$ and $(T, G)$ is not maximal, then there exists a unique 2-marking $\phi$ of $T$ such that $\phi\left(H^{2}(T, \mathbb{Z})^{G}\right)=M_{\mathfrak{x}}$ and $\phi\left(H^{2}(T, Z)_{G}\right)=L_{\mathfrak{q}}$. Moreover, $\phi$ induces an isomorphism of $\bar{G}$ with the unique normal subgroup of order 4 of $S H\left(L_{\Theta}\right) \cong \mathfrak{U}_{4}$. 3) If $G=\mathfrak{夭}_{2}$, $H^{2}(T, Z)_{G}=\{0\}$ and $\bar{G}=\{e\}$.

Proof. Set $L=L_{\circledast}$ and $H=S H\left(L_{\circledast}\right)$. Then by (29) $L^{H}=0$. Hence if we set $M=M_{\text {क }},(L, H, E) \in \mathscr{B}$ for any $E \in V_{M} \neq \emptyset$. Let $\left(T^{\prime}, G^{\prime}\right)$ be a special pair with $-1 \in G^{\prime}$ corresponding to such an $(L, H, E)$. Then by (29) and the relation $G^{\prime} /\langle-1\rangle \cong H$, we see that the orders of $G^{\prime}$ and \&s are the same. If follows that $G^{\prime} \cong \mathbb{S}$. Further, by Lemma 6.2,3) we conclude that $\left(T^{\prime}, G^{\prime}\right)$ is maximal if $\mathbb{G}=\mathfrak{Q}$. 1) follows from this, in view of the remark at the beginning of this subsection. 2) follows from 1) immediately, and 3) is obvious.

Remark. By extending elements of $H=S H\left(L_{\circledast}\right)$ uniquely to $\operatorname{SO}\left(U^{3}\right)$ by Lemma 6.1 we consider $H$ as a subgroup of $\mathrm{SO}\left(U^{3}\right)$. As we have noted $H\left(L_{\oplus}\right)$ is the Weyl group of the corresponding root system; further $S H\left(L_{\circledast}\right)$ is the subgroup of those elements of even length (with respect to generators consisting of reflections). From this we see that any element of $S H\left(L_{\circledast}\right)$ has spinorial norm 1 (cf. $[16, \S 55]$ ) so that actually we have $H \subseteq \mathrm{SO}_{0}\left(U^{3}\right)$. Using this fact one can give a more direct proof (i。 $e_{\circ}$, the one which is independent of the results 
of Section 2) of the fact that $(T, G)$ is special in the last part of the proof of Proposition 6.3.

Corollary 6.5. There exists up to equivalence a unique primitive embedding of $L_{\oplus}$ into $U^{3}$.

Proof. Let $L$ be any primitive sublattice of $U^{3}$ isomorphic to $L_{\circledast}$. Let $H=S H(L)$. Then by repeating the argument of the proof of the theorem we see that there exists a special pair $(T, G)$ which is in the case 1) of Theorem 6.4 with invariant $(L, H)$. It follows from the theorem that $(L, H)$ is equivalent to $\left(L_{\circledast}, S H\left(L_{\circledast}\right)\right)$. Since $L_{\circledast}^{\frac{1}{3}} \cong M_{\text {G, }}$ any element of $\mathrm{O}\left(L_{\mathrm{G}}\right)$ extends to an element of $\mathrm{O}\left(U^{3}\right)$. It follows that the embedding itself is unique.

Remark 6. 6. 1) More precicely, we can always find an element $u \in \mathrm{SO}_{0}\left(U^{3}\right)$ which sends $L$ onto $L_{0}$. This follows from the existence of elements $v_{1} \in \mathrm{O}\left(U^{3}\right)-\mathrm{SO}\left(U^{3}\right)$ and $v_{2}=-\mathrm{id}_{U^{3}} \in \mathrm{SO}\left(U^{3}\right)-\mathrm{SO}_{0}\left(U^{3}\right)$ leaving $L_{\circledast}$ invarinat. (For $v_{1}$ take-for instance the extension of any element of $H\left(L_{\circledast}\right)-S H\left(L_{\circledast}\right)$ which is the identity on $M_{\circledast}$.)

2) If $\mathbb{S}=\mathbb{C}_{m}$, the corollary is a special case of the general uniqueness theorem of Nikulin (cf. $[15,1.12 .3]$ ). There exists a direct proof also in the case $\mathscr{B} \neq \mathbb{E}_{m}$ (cf. Remark 7.18).

Similarly we obtain from Theorem 6.4 the following:

Corollary 6.7. 1) Among all the binary positive even lattices $L, A_{1}^{2}$ and $A_{2}$ are characterized by the condition that $\left.S H(L) \neq\{e\} .2\right)$ Among all the ternary positive even lattices $L, A_{1}^{3}, A_{1} \perp A_{2}$, and $A_{3}$ are characterized by the condition that $S H(L)$ is non-cyclic.

Remark 6.8. Actually 1) is immediate from the well-known conformal classification of binary positive lattices (cf. [2, II, §7]). 2) could also have been obtained from the classical Braviais classification of space lattices.

6.5. Next we shall give a characterization of those complex tori $T$ which admit a special action of $\mathbb{S}$ as in 6.4 in terms of its NeronSeveri lattice $N_{T}$ or of its transcendental lattice $B_{T}$ 。 
Theorem 6.9. Let $T$ be a complex torus of dimension 2. Let (S) $=\mathfrak{C}_{m}, m=4,6, \mathfrak{Q}, \mathfrak{I}$, or $\mathfrak{D}$. Then the following conditions are equivalent. 1) There exists a finite subgroup of $G$ of Aut $T$ with $G \cong \mathbb{S}$ such that the resulting pair $(T, G)$ is special, and in addition, is maximal if $\circlearrowleft \mathfrak{=}$. 2) There exists a primitive embedding $L_{\Theta} \longrightarrow N_{T}$. 3) There exists a primitive embedding $B_{\mathfrak{G}} \longrightarrow M_{T}$.

Remark 6. 10. The following three assertions are clear: 1) Any complex torus of dimension 2 admits a unique special action of $\mathfrak{F}_{2}$. 2) $T$ admits a special action of $\mho_{3}$ if and only if it admits a special action of $\mathfrak{S}_{6}$. 3) $T$ admits a special and non-maximal action of $\mathscr{Q}$ if and only if it admits a special action of $\mathfrak{T}$ (cf. 3.3). Hence the above theorem gives a complete characterization of complex tori which admits a special action of a given finite group.

Remark 6.11. In case $\mathbb{S}=\mathfrak{Q}$, $\mathfrak{T}$, or $\mathfrak{D}$ we have the two alternative cases: a) $a(T)=0, \rho(T)=3, B_{T} \cong M_{\circledast}$ and $N_{T} \cong L_{\circledast}$ : in particular in this case $N_{T}$ and $B_{T}$ are independent of the isomorphism classes of $T$. b) $T$ is a singular abelian surface and $B_{T}$ is primitively embedded in $M_{\oplus}=L_{\oplus}(-1)$.

Proof of Theorem 6.9. 1) implies 2) by Theorem 6.4 and Lemma 6. 2. Let $L=L_{\circledast}$ and $M=M_{\oplus}$. Suppose now that 2) is true. Let $u: L \longrightarrow N_{T}$ be any primitive embedding. Then by Corollary 6.5 (cf. Remark 6.6 also) we can find an admissible 2-marking $\psi$ of $T$ such that $\phi(u(L))=L$. Let $E=E(T, \phi)$ and $H=S H(L)$. Then by Proposition 6.3 there exists a finite subgroup $G$ of Aut $T$ such that $(T, G)$ is special, $-1 \in G$ and $\beta((T, G ; \psi))=(L, H, E)$. Thus 1) follows. On the other hand, 2) implies the existence of an embedding $B_{T} \longrightarrow L^{\perp}$, while by Corollary 6.5 $L^{\perp} \cong M$. Hence 3 ) is true. Finally we shall show that 3 ) implies 2). Let $B_{T} \longrightarrow M$ be any primitive embedding. This induces a primitive embedding $N^{\perp \longrightarrow} N_{T}$. If $\& \& \mathbb{E}_{m}$, then by Corollary 6.5 $M^{\perp} \cong L_{\circledast}$ and 2) is true. If $(S)=\mathbb{C}_{m}$, then $\left|\operatorname{discr} M^{\perp}\right|=$ $|\operatorname{discr} M|=\left|\operatorname{discr} L_{\circledast}\right|=4$ if $m=4$ and $=3$ if $m=6$, where discr denotes the discriminant. As is well-known, binary negative even lattice with discriminant -4 or -3 is unique (cf. [2]). Hence $M^{\perp} \cong L_{\circledast} ;$ so 2 ) is true in this case also. 
We refine the above theorem slightly in the case of singular abelian surfaces. Let $T$ be a singular abelian surface. Then $\operatorname{rank} B_{T}=2$; hence we have $E(T)=B_{T, R}$. In particular $B_{T}$ has the natural induced orientation. Let $(T, G)$ be a special pair with $-1 \in G$ and with $T$ a singular abelian surface. Take any isomorphism $u: H^{2}(T, \mathbb{Z})^{G} \rightarrow M_{\mathscr{B}}$, which in turn induces a primitive embedding $u_{0}: B_{T} \longrightarrow M_{\Theta}$ (cf. Theorem 6. 4 and Lemma 6.2). Let $B(T, G)$ be the image of $u_{0}$. We put on $B(T, G)$ the orientation induced from $B_{T}$. We say that the oriented sublattices $V_{1}$ and $V_{2}$ of $M_{\mathfrak{G}}$ are equivalent if there exists an element $\mu \in \mathrm{O}\left(M_{\circledast}\right)$ such that $\mu\left(V_{1}\right)=V_{2}$ with the induced isomorphism $V_{1} \rightarrow V_{2}$ orientation preserving. Then the equivalence class of the oriented sublattices $B(T, G)$ is independent of the choice of $u$ as above and depends only on the pair $(T, G)$.

Proposition 6.12. Let \&S be as in Theorem 6.9. The correspondence $(T, G) \rightarrow B(T, G)$ defined above sets up a natural bijective correspondence between a) the set $\hat{\mathscr{S}}$ of equivalence classes of special pairs $(T, G)$ with $G \cong \mathbb{S}$, with $T$ a singular abelian surface and with $(T, G)$ maximal if $G \cong \mathfrak{Q}$, and $b$ ) the set $\mathscr{V}$ of oriented primitive binary positive sublattices of $M_{\circledast}$ considered up to equivalence.

Proof. Injectivity. Let $i=1,2$. Let $\left(T_{i}, G_{i}\right)$ be pairs from $\hat{\mathscr{S}}$. Suppose that $B\left(T_{i}, G_{i}\right)$ are equivalent sublattices of $M_{\circledast}$ 。 Let $u$ be an element of $\mathrm{O}\left(M_{\circledast}\right)$ which gives an equivalence as above. Let $\left(L_{i}, H_{i}, E_{i}\right)$ be the triples corresponding to $\left(T_{i}, G_{i} ; \psi_{i}\right)$ for some admissible 2-markings $\phi_{i}$ for $\left(T_{i}, G_{i}\right)$ via Proposition 6.3. Then by that proposition it suffices to show that these triples are equivalent. Since $\& \cong \cong G_{1} \cong G_{2}$ and $\left(T_{i}, G_{i}\right)$ are maximal if $G_{i} \cong \mathfrak{Q}$, by Theorem 6.4 if we choose $\phi_{i}$ suitably we may assume that $L_{1}=L_{2}=L_{\circledast} \subseteq U^{3}$ and $H_{1}=H_{2}=S H\left(L_{\circledast}\right)$. Now using Lemma 6.1 we extend $u$ to an element $\tilde{u}$ of $\mathrm{SO}\left(U^{3}\right)$. Further, by taking $-u$ instead of $u$ if necessary, we may assume that $\tilde{u} \in S O_{0}\left(U^{3}\right)$. Then $\tilde{u}$ preserves $L_{G}$ and sends $E\left(T_{1}, \psi_{1}\right)=B\left(T_{1}, G_{1}\right)_{R}$ onto $E\left(T_{2}, \psi_{2}\right)=B\left(T_{2}, G_{2}\right)_{R}$ orientation preservingly. Hence $\left(L_{i}, H_{i}, E_{i}\right)$ are isomorphic as desired.

Surjectivity. Let $B$ be an oriented primitive binary positive subla-

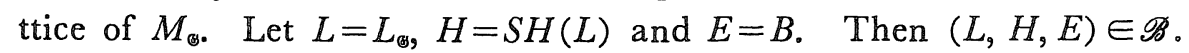
Let $(T, G ; \psi)$ be an admissible 2-marked special pair corresponding 
to the triple $(L, H, E)$ by Proposition 6.3. Then by construction $B=B(T, G)$ (modulo equivalence). By Theorem $6.4(T, G)$ is maximal if $G \cong \mathfrak{Q}$. It remains to show that $T$ is a singular abelian surface. Let $N=B^{\perp}$. Then $N$ is of rank 4 and is orthogonal to the period $E=E(T, \psi)$ of $(T, \psi)$. This implies that $\psi^{-1}(N) \subseteq N_{T}$. Hence $\rho(T)=$ rank $N_{T} \geqq 4$; thus $T$ is a singular abelian surface.

6. 6. Let $\&$ be $\mathfrak{Q}, \mathfrak{I}$, or $\mathfrak{D}$. Let $(T, G)$ be a maximal special pair

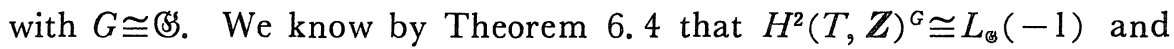
$H^{2}(T, Z)_{G}=L_{\oplus}$ as euclidian lattices. However, the proof there of this fact was rather conceptual but not explicit. So we shall exhibit a more explicit isomorphism here, by using the calculation given in the last part of Section 5. The method also gives us the proof of Proposition 3. 15.

Recall that by Lemma 5.8 we have the natural $G$-equivariant isometry

$$
f: H_{0} \perp H_{0}(-1) \rightarrow H^{2}(T, Q) \text {. }
$$

(See below for more explicit formula.) Here the notation $H_{0}$ is explained as follows. Let $H$ be the definite quaternion algebra given by

$$
H=\mathbb{Q}[1, i, j, k] \text { if } \mathscr{S}=\mathfrak{Q} \text { or } \mathfrak{T} \text { and }=\mathbb{Q}[1, i, \sqrt{3} j, \sqrt{3} k] \text { if } \mathbb{S}=\mathfrak{D}
$$

as a $\boldsymbol{Q}$-subalgebra of $\boldsymbol{H}$. Then $H_{0}$ is the $\boldsymbol{Q}$-subspace of pure quaternions of $H$ endowed with the inner product defined by $\langle x, y\rangle=\operatorname{tr}(x \bar{y})$ (cf. 5.4). We have also fixed an identification $T=\boldsymbol{H} / \Lambda$ as an oriented real torus, where $\Lambda=\Lambda_{\mathbb{B}} \quad$ (cf. (16)) and we consider elements of $H^{2}(T, Q)$ as $\boldsymbol{Q}$-valued alternating forms on $H \subseteq \boldsymbol{H}$. Now let

$$
\hat{L}=f^{-1}\left(H^{2}(T, Z)\right) \subseteq H_{0} \perp H_{0}(-1) .
$$

Let $L=\hat{L} \cap H_{0}$ and $L^{\prime}=\hat{L} \cap H_{0}(-1)$. Then

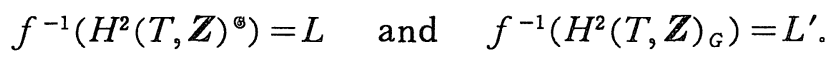

Therefore our task is to exhibit an explicit isomorphism $L \cong L_{\circledast}(-1)$ and $L^{\prime} \cong L_{\Theta}$ as euclidian lattices.

Recall first that for any $\xi \in H_{0}$

$$
f(\xi)=(1 / \sqrt{d}) \operatorname{tr}(x \xi \bar{y})
$$

for any $x, y \in H_{0}$, where $d=d_{\circledast}$ is given respectively by $d_{\Omega}=4, d_{\nwarrow}=2$ and $d_{\triangleright}=3$ (cf. (23)). Hence $\xi \in L$ if and only if $(1 / \sqrt{d}) \operatorname{tr}(x \xi \bar{y}) \in \mathbb{Z}$ 
for any $x, y \in \Lambda$. Hence if we set

$$
L_{1}=\{\zeta \in H ; \operatorname{tr}(x \zeta \bar{y}) \in \mathbb{Z} \text { for any } x, y \in \Lambda\},
$$

then $L=\sqrt{d} L_{1} \cap H_{0}$. On the other hand, for any $\zeta \in H, \zeta \in L_{1}$ if and only if $\zeta \bar{y} \in \Lambda^{*}$ for any $y \in \Lambda$, where $\Lambda^{*}:=\{z \in H ; \operatorname{tr}(z x) \in \mathbb{Z}$ for any $x \in \Lambda\}$ is the dual lattice of $\Lambda$ with respect to the nondegenerate bilinear form $\operatorname{tr}(x z)$.

Proposition 6.13. The notations being as above, $L$ is given by 1$) \Lambda \cap H_{0}$ if $\mathbb{S}=\mathfrak{Q}, 2)((1+i) / \sqrt{2}) \Lambda) \cap H_{0}$ if $\mathbb{S}=\mathfrak{T}$ and 3$)(((\sqrt{3}+k) / 2) \Lambda) \cap H_{0}$ if $\mathbb{S}=\mathfrak{D}$. In each case we can take a $\mathbb{Z}$-basis of $L$ as follows; 1) $i, j, k, \quad 2)(i+j) / \sqrt{2},(j+k) / \sqrt{2},(k+i) / \sqrt{2}$ and 3$)(\sqrt{3} i+j) / 2$, $(\sqrt{3} i-j) / 2, k$.

Proof. By straightforward computation we get that $\Lambda^{*}=z \Lambda=\Lambda z$, where $z=z_{\circledast}=1 / 2,(1+i) / 2,(3+\sqrt{3} k) / 6$ according as $\mathscr{G}=\mathfrak{O}, \mathfrak{I}$ and (I) respectively. Then, since $\Lambda=\bar{\Lambda}$, for any $\zeta \in H, \zeta \in L_{1}$ if and only if $\zeta y \in z \Lambda$ for any $y \in \Lambda$. On the other hand, since $\Lambda$ is an order, $\zeta y \in z \Lambda$ for any $y \in \Lambda$ if and only if $\zeta \in z \Lambda$. Therefore $L_{1}=z \Lambda$ and $L=(\sqrt{d} z \Lambda) \cap H_{0}$ 。 Substituting the values of $z=z_{\Theta}$ and $d=d_{\Theta}$ we get the description of $L$ in the proposition. It is immediate to check that the three elements of the proposition form a $\mathbb{Z}$-basis of $L$ in each case.

Corollary 6.14. Let $L_{(1)}=\{\xi \in L ; n(\xi)=1\}$, where $n(\xi)$ is the norm of $\xi$. Then $L_{(1)}$ is given by 1$)\{ \pm i, \pm j, \pm k\}$ if $\left.\mathbb{S}=\mathfrak{\Omega}, \quad 2\right)\{(1 / \sqrt{2})$ $( \pm i \pm j),(1 / \sqrt{2})( \pm i \pm k),(1 / \sqrt{2})( \pm j \pm k)\}$ if $\mathbb{B}=\mathfrak{I}$ and 3$)\{( \pm \sqrt{3} i \pm j) / 2$ $\pm j, \pm k\}$ if (s) $=\mathfrak{D}$.

Proof. By Proposition 6.13 any element $\xi$ of $L$ can be written as $\xi=w \zeta$ for some $\zeta \in \Lambda$, where $w=1,(1+i) / \sqrt{2}$ or $(\sqrt{3}+k) / 2$. Since $n(w)=1, n(\xi)=1$ if and only if $n(\zeta)=1$. Hence by Proposition 6.13 we get $L_{(1)}=w \Lambda^{\times} \cap H_{0}$, where $\Lambda^{\times}$is the unit group of the order $\Lambda$. From this the corollary follows immediately (cf. (16) and Lemma 2.6).

The "intersection matrix" of $L$ with respect to the $\mathbb{Z}$-basis of Proposition 6.13 are as follows; 

1) $\left(\begin{array}{lll}2 & 0 & 0 \\ 0 & 2 & 0 \\ 0 & 0 & 2\end{array}\right)$
2) $\left(\begin{array}{lll}2 & 1 & 1 \\ 1 & 2 & 1 \\ 1 & 1 & 2\end{array}\right)$
3) $\left(\begin{array}{rrr}2 & -1 & 0 \\ -1 & 2 & 0 \\ 0 & 0 & 2\end{array}\right)$.

It follows that $L(-1)$ is isomorphic to $A_{1}^{3}, A_{3}$ and $A_{2} \perp A_{1}$ (i. e., to $\left.L_{6}\right)$, in respective cases as desired. Indeed, in case 2), passing to another $\boldsymbol{Z}$-basis, say $(i+j) / \sqrt{2},-(j+k) / \sqrt{2},(j-i) / \sqrt{2}$, the corresponding matrix becomes the Cartan matrix of $A_{3}$. The isomorphism $L^{\prime} \cong L_{\text {ब }}$ can be shown in the same way. Finally using Corollary 6.14 we shall give the:

Proof of Proposition 3.15. Let $(T, G)$ be a maximal special pair. By Theorem 3.11 we may assume that $(T, G)=\left(T_{q}, G_{q}\right)$ for some $q \in X$. Now $T$ admits a $G$-invariant principal polarization if and only if the $G$-invariant Neron-Severi lattice $N_{T}^{G}$ contains an element $\xi$ of length 2. On the other hand, $N_{T}^{G}=L \cap \boldsymbol{R} q$ in $H_{0}$, where $L$ is as in Proposition 6.13 (cf. §5). Hence the condition is further reduced to: $L_{(1)} \cap \boldsymbol{R} q \neq \emptyset$, or, since the norm of $q$ equals 1 , to: $q \in L_{(1)}$. From the explicit description of $L_{(1)}$ in Corollary 6.14 we see that $L_{(1)}$ consists of a single $\Gamma_{\mathfrak{Q}}^{\times}$-orbit if $\mathfrak{S}=\mathfrak{Q}$ or $\mathfrak{I}$ and consists of two orbits $\{( \pm \sqrt{3} i+j) / 2, \pm j\}$, and $\{ \pm k\}$. From this the first assertion of the proposition follows. The uniqueness of the polarization then follows from Corollary 5. 11 .

Let $K$ be the normalizer of $G$ in Aut $T$. Then $K$ is finite (cf。 2. 5) so that the $K$-invariant Neron-Severi lattice $N_{T}^{K}$ of $T$ is non-zero. Then from the inclusion $N_{T}^{K} \subseteq N_{T}^{G} \cong \mathbb{Z}$ we have $N_{T}^{K}=N_{T}^{G}$. Hence $K$ also fixes the principal polarization under consideration.

\section{§ 7. Special Pairs for Singular Abelian Surfaces}

7. 1. Singular abelian surfaces are in one-to-one correspondence with binary positive even lattices via their transcendental lattices (Shioda-Mitani [23]). On the other hand, by Proposition 6.12 the existence of a special action of a finite group \&s as in 6.4 on a singular abelian surface is reduced to the existence of a primitive embedding of the corresponding binary positive even lattice into $L_{\circledast}$. By making the latter condition more precise we can obtain a more detailed description of the special pairs $(T, G)$ with $G \cong \mathbb{S}$ and with $T$ a singular 
abelian surface.

First, in order to fix notations and terminologies, and also for the convenience of the reader we shall summarize the main results of [23] mentioned above.

a) Let $(A, q)$ be a finite quadratic form group. For any prime number $p$ let $A_{p}$ be the $p$-torsion part of $A_{\text {. }}$ Let $q_{p}=q_{\mid A_{p}}$ be the restriction of $q$ to $A_{p}$. Then we have the direct sum decomposition of the quadratic form group $(A, q)=\oplus_{p}\left(A_{p}, q_{p}\right)$.

For any integers $n, \lambda$ with $n>1$ and with $n \lambda$ even we define the quadratic form group $K_{\lambda}(n)$ by

$$
K_{\lambda}(n)=(\mathbb{Z} / n \mathbb{Z},(\lambda / n)) 。
$$

Also we define

$$
\begin{aligned}
& U_{k}=\left(\left(\mathbb{Z} / 2^{k} \mathbb{Z}\right)^{2}, \quad\left(\begin{array}{ll}
0 & 2^{-k} \\
2^{-k} & 0
\end{array}\right)\right), k \geqq 1 \\
& V_{k}=\left(\left(\mathbb{Z} / 2^{k} \mathbb{Z}\right)^{2}, \quad\left(\begin{array}{ll}
2^{-k+1} & 2^{-k} \\
2^{-k} & 2^{-k+1}
\end{array}\right)\right), k \geqq 1 .
\end{aligned}
$$

For simplicity we write $K(n)$ for $K_{1}(n)$ and set $K_{\lambda}(1)=(\{0\}, 0)$ for any $\lambda$. Any indecomposable quadratic form group is isomorphic to one of $K_{\lambda}\left(p^{k}\right), U_{k}$ and $V_{k}$, where $p$ is any prime, $(\lambda, p)=1$ and $k \geqq 1$ (cf. Nikulin [15]).

Let $n$ be odd and $l=n$ or $2 n$. Then we have

$$
\mathrm{O}\left(K_{\lambda}(l)\right) \cong \mathfrak{E}_{2}^{s},
$$

where $s$ is the number of distinct prime factors of $n$.

b) Let $m$ be a square-free positive integer. Then we set $K_{m}=$ $Q(\sqrt{-m})$. Let $d=d_{m}$ be the negative of the discriminant of $K_{m}$. Then $d$ is given by

$$
d=4 m \text { if } m \equiv 1,2(4) \text {, and }=m \text { if } m \equiv 3 \text { (4). }
$$

Define $\omega=\omega_{m}$ by $\omega=\sqrt{-m}$ if $m \equiv 1,2$ (4) and $=(-1+\sqrt{-m}) / 2$ if $m \equiv 3$ (4). Then any order $D$ contained in $K_{m}$ is of the form $\mathrm{D}=\mathrm{o}_{f}:=\mathbb{Z}+\mathbb{Z} f \omega$ for a unique positive integer $f$, called the conductor of o. A proper $0_{f}$-ideal is a free $\mathbb{Z}$-submodule $M$ of $K_{m}$ of rank 2 such that $\mathrm{o}_{f}=\left\{\alpha \in K_{m} ; \alpha M \subseteq M\right\}$. Two proper $\mathrm{o}_{f}$-ideals $M$ and $M^{\prime}$ are said to be equivalent if there exists an element $\alpha \in K_{m}$ such that $\alpha M=M^{\prime}$. The set of equivalence classes, denoted by $\mathscr{I}_{m, f}$, has the natural structure 
of a finite abelian group.

c) Let $L$ be a binary positive even lattice. Let $D>0$ be the discriminant of $L$. Then $\boldsymbol{Q}(\sqrt{-D})$ is an imaginary quadratic field and hence is isomorphic to $K_{m}$ for a unique squarefree positive integer $m=m_{L}$. Then we may write

$$
D=d f^{2}
$$

for a unique positive integer $f$, called the conductor of $L$, where $d=d_{m}$. With respect to any basis we may represent $L$ by a $2 \times 2$ integral matrix

$$
\left(\begin{array}{rr}
2 a & b \\
b & 2 c
\end{array}\right)
$$

with $D=4 a c-b^{2}$. Let $s=$ G. C. D. $(a, b, c)$. Then $s$ is independent of the choice of the basis and called the degree of primitivity of $L$. We say that $L$ is primitive if $s=1$. For any $L$ there exists a unique primitive binary positive even lattice $L_{0}$ such that

$$
L \cong L_{0}(s) \text {. }
$$

In this case we call the conductor $f_{0}$ of $L_{0}$ the reduced conductor of $L$. Thus to any binary positive even lattice we have associated a triple of numerical invariants

$$
\left(m, f_{0}, s\right) \text {, }
$$

by which the conductor $f$ and the discriminant $D$ of $L$ are recovered respectively by:

$$
f=s f_{0} \quad \text { and } \quad D=d s^{2} f_{0}^{2},
$$

where $d$ is determined by $m$ via (30). For a primitive lattice $L_{0}$ this reduces to a pair of invariants $(m, f)$.

Let $\mathscr{L}$ (resp. $\mathscr{L}_{0}$ ) be the set of isomorphism classes of binary positive even lattices (resp. primitive such lattices). Let $N$ be the set of natural numbers. Then by the isomorphism (33) we have the natural bijection

$$
\mathscr{L} \cong \mathscr{L}_{0} \times N
$$

d) Let $L, L^{\prime} \in \mathscr{L}$. Then we say that $L$ and $L^{\prime}$ are in the same genus if for any prime $p$ the $p$-adic lattices $L \otimes_{Z} Z_{p}$ and $L^{\prime} \otimes_{Z} Z_{p}$ are isomorphic, where $\mathbb{Z}_{p}$ is the ring of $p$-adic integers. We call such an equivalence class a genus. Nikulin $[15,1.9 .4]$ proved the following: 
i) $L$ and $L^{\prime}$ are in the same genus if and only if the discriminant forms of $L$ and $L^{\prime}$ are isomorphic to each other.

In this connection we also note the following:

ii) The invariant $\left(m, f_{0}, s\right)$ of a binary positive even lattice $L$ depends only on the genus to which $L$ belongs, i. e., $\left(m, f_{0}, s\right)$ is an invariant of a genus.

Proof: For any prime $p$, the $p$-component $\left(A_{p}, q_{p}\right)$ of the discriminant form $(A, q)$ of $L$ is of one of the following forms: $K_{\lambda_{p}}\left(p^{k} p\right) \oplus$ $K_{\theta_{k}}\left(p^{l_{p}}\right), 0 \leqq k_{p} \leqq l_{p}, p \geqq 2$, or $V_{k_{2}-1}$ or $U_{k_{2}-1}, k_{2} \geqq 2$ when $\left.p=2\left(\mathrm{cf}_{\circ} a\right)\right)$. In this case $s$ is simply given by $s=2^{k_{2}-1} \prod_{p \text { odd }} p^{k_{p}}$. On the other hand, by (26) the discriminant $D$ is determined by $A$. Then from the relations (31) and (34) $f_{0}$ and $m$ can be recovered from $D / s^{2}$.

e) Let $m$ and $f$ be positive integers with $m$ squarefree. Then we set

$\mathscr{L}_{m, f}=$ \{oriented primitive binary positive even lattice with invariant $(m, f)\} / \sim$,

$\mathscr{E}_{m, f}=\left\{\right.$ elliptic curves $E$ with $\operatorname{End}_{0} E \cong K_{m}$ and End $\left.E \cong \mathfrak{o}_{f}\right\} / \sim$, where $\sim$ denotes "up to isomorphisms". Then the following is classical (cf. e.g. [2, II, §7] and [21, 4.8]).

Proposition 7.1. There exist natural bijective correspondences among the three sets $\mathscr{L}_{m, f}, \mathscr{I}_{m, f}$, and $\mathscr{E}_{m, f}$.

We shall briefly recall the correspondences $\mathscr{I}_{m, 5} \rightarrow \mathscr{L}_{m, f}$ and $\mathscr{I}_{m, f} \rightarrow$ $\mathscr{E}_{m, f}$. Fix an embedding $j: K_{m} \longrightarrow \mathbb{C}$. Let $C \in \mathscr{I}_{m, f \circ}$ Choose a representative $M$ of $C$ such that $M \cong \mathrm{o}_{f}$. Since $M \otimes \mathbb{R} \cong \mathbb{C}$ via $j, M$ is given a natural orientation. On the other hand, the restriction of the bilinear form $\operatorname{tr}_{K_{m} / \theta}(x \bar{y}), x, y \in K_{m}$, on $M$ makes $M$ into a binary positive even lattice. Then we associate to $C 1$ ) the primitive even lattice $M_{0} \in \mathscr{L}_{m, f}$ associated to $M$ via (33) and 2) the elliptic curve $E:=\mathbb{C} / M_{0} \in \mathscr{E}_{m, f}$, where we consider $M_{0} \subseteq \mathbb{C}$ via $j$.

f) (cf. [2, III, §8]) Via the above correspondences $\mathscr{L}_{m, f}$ is given the natural structure of a finite abelian group since $\mathscr{I}_{m, f}$ is one. It is then a classical fact that

i) a genus of binary positive primitive even lattices with invariant $(m, 1)$ corresponds to a coset of the quotient group $\mathscr{L}_{m, 1} / \mathscr{L}_{m, 1}^{2}$ bijecti- 
vely, where $\mathscr{L}_{m, 1}^{2}=\left\{M^{2} ; M \in \mathscr{L}_{m, f}\right\}$. (For any $L$ and $L^{\prime} \in \mathscr{L}_{m, f}$ it is known that $L$ and $L^{\prime}$ are in the same genus if and only if $L \otimes Q$ and $L^{\prime} \otimes \boldsymbol{Q}$ are isomorphic over $\boldsymbol{Q}$.) It is also known that

ii) if $t$ is the number of positive distinct prime factors $d$, then $\mathscr{L}_{m, 1} / \mathscr{L}_{m, 1}^{2}$ is an elementary abelian 2-group of rank $t-1$.

In particular,

iii) the following conditions are equivalent: 1) There exists a unique genus of primitive lattices with invariant $(m, 1), 2)$ the class number of $K_{m}$ is odd and 3) $m=1,2$, or $m$ is prime with $m \equiv 3$ (4).

g) For any square-free positive integer $m$ we define the sets $\mathscr{S}_{m}$, $\mathscr{E}_{m}$, and $\mathscr{L}_{m}$ as follows;

$\mathscr{S}_{m}=\left\{\right.$ singular abelian surfaces $T$ with $\left.m_{T}=m\right\} / \sim$,

$\mathscr{E}_{m}=$ \{elliptic curves $E$ with $\left.\operatorname{End}_{0} T \cong K_{m}\right\} / \sim$,

$\mathscr{L}_{m}=$ \{oriented binary positive even lattice $L$ with $\left.m_{L}=m\right\} / \sim$,

where $\sim$ means "up to isomorphisms" and see Sect. 6 for $m_{T}$. Then the following holds.

Theorem (Shioda-Mitani [23]). There exist natural bijective correspondences among the following three sets;1) $\left.\mathscr{S}_{m}, 2\right) \mathscr{E}_{m} \times N$ and 3$) \mathscr{L}_{m}$, where $N$ is the set of natural numbers.

We shall briefly recall the correspondences of the theorem.

1) $\rightarrow 2)$. Let $T \in \mathscr{S}_{m}$. Then $E:=H^{2}\left(T, \mathcal{O}_{T}\right) / \operatorname{Im} H^{2}(T, \mathbb{Z})$ is an elliptic curve in $\mathscr{E}_{m}$. Let $\mathfrak{o}_{f}=$ End $E \cong \operatorname{End}_{0} E \cong K_{m}$. Let $\mathfrak{o}_{f}$, be the center of End $T$. Then $\mathfrak{o}_{f} \cong \mathfrak{o}_{f}$ so that $f$ divides $f^{\prime}$. Then we associate to $T$ the pair $\left(E, f^{\prime} / f\right)$.

$2) \rightarrow 1)$. Let $(E, s) \in E_{m} \times N$. Let $\mathfrak{o}_{f}=$ End $E$. Then $E^{\prime}:=C / \mathfrak{o}_{f s}$ is an elliptic curve with End $E^{\prime} \cong \mathfrak{D}_{f s}$. Then we associate to $(E, s)$ the singular abelian surface $T=E \times E^{\prime}$.

$2) \rightarrow 3$ ). The correspondence is obtained via (35) and Proposition 7.1.

1) $\rightarrow 3$ ). To each $T \in \mathscr{S}_{m}$ we associate its transcendental lattice $B_{T}$ with its natural orientation.

3) $\rightarrow 1$ ). Let $L \in \mathscr{L}_{m}$. Represent $L$ by a $2 \times 2$ integral matrix as in (32) with respect to an oriented base. Then we associate to $L$ the singular abelian surface 


$$
T=E_{\tau} \times E_{\tau^{\prime}}
$$

where $\tau=(-b+\sqrt{-D}) / 2 a$ and $\tau^{\prime}=(b+\sqrt{-D}) / 2$.

We note that the second set $\mathscr{E}_{m} \times \mathbb{N}$ is not explicit in [23] but one can deduce the validity of the above correspondences readily from [23, Prop. 4.5]. In particular we see that the presentation (36) of $T$ as a product of elliptic curves is canonical in the sense that the elliptic curves are determined intrinsically by $T$.

7. 2. Let $T$ be a singular abelian surface with transcendental lattice $B_{T}$. Then we shall say that $T$ is with invariant $\left(m, f_{0}, s\right)$ (resp. primitive) if so is $B_{T}$ (cf. 7.1, c)). Similarly, if $\mathscr{G}$ is a genus of a binary positive even lattices, then we say that $T$ belongs to $\mathscr{G}$ if $B_{T}$ does.

Theorem 7.2. Let $\mathbb{B}=\mathfrak{Q}$ or $\mathfrak{I}$. Let $(T, G)$ be a maximal special pair with $G \cong \mathbb{S}$. Suppose that $T$ is a singular abelian surface with invariant $\left(m, f_{0}, s\right)$. Then $m, f_{0}, s$ satisfy the following conditions; (i) $m \not \equiv-1$ (8), $i_{\circ} e_{0}, m \equiv 1,2$ (4) or $m \equiv 3$ (8), (ii) $f_{0}$ is odd, and (iii) $s=2$ if $\mathbb{S}=\mathscr{Q}$ and if $m \equiv 3$ (8); otherwise $s=1$. Conversely, if positive integers $m, f_{0}, s$ with $m$ square-free satisfy these conditions, then among all the genera with invariant $\left(m, f_{0}, s\right) \quad\left(c f_{0} 7.1, \mathrm{~d}\right)$, ii)) there exists a unique genus $\mathscr{G}=\mathscr{G}_{m, f_{0}, s}$ such that a singular abelian surface $T$ with invariant $\left(m, f_{0}, s\right)$ admits a maximal special action of \&s if and only if $T$ belongs to the genus $\mathscr{G}$; moreover $\mathscr{G}$ is explicitly given via the corresponding discriminant form $(A, q)(c f .7 .1, \mathrm{~d}) \mathrm{i}))$ as in Table 16 below.

Corollary 7. 3. If either $m=1,2$ or $m$ is a prime with $m \equiv 3$ (8), then any primitive singular abelian surface $T$ with invariant $(m, 1)$ always admits a special action of $\mathfrak{I}$.

For instance $E_{\omega} \times E_{\omega}, \omega=\omega_{m}$, admits a special action of $\mathfrak{T}$ if $m$ satisfies the above condition. The corollary can also be deduced directly from a theorem of Steinitz on the structure of a finite torsionfree module over a Dedekind domain (cf. $[17 ;$ p. 48]).

Let $\left(m, f_{0}, s\right)$ satisfy the conditions (i)-(iii) of the above theorem. Let $T$ be a singular abelian surface belonging to the genus $\mathscr{G}=\mathscr{G}_{m, f_{0}, s}$ of the theorem so that $T$ admits a maximal special action of $\mathbb{S}$. Fix 
such an action and let $(T, G)$ be the resulting pair. Let $\delta$ be the degree of $(T, G)$ as was defined before Remark 5.12.

Theorem 7.4. 1) $\delta$ depends only on the genus $\mathscr{G}$, and is independent of $T$ and of the chosen action of $\mathbb{S} ;$; indeed $\delta$ is explicitly given for each (S) in Table 16 below. 2) Let $t$ be the number of distinct odd prime factors of $\delta$. Then the number of conjugacy classes of maximal special subgroups of Aut $T$ which is isomorphic to \&s equals $2^{t-1}$.

Table 16

\begin{tabular}{|c|c|c|c|c|}
\hline \&s & $m$ & $s$ & $(A, q)$ corresponding to $\mathscr{G}_{m, f_{0}, s}$ & $\delta$ \\
\hline \multirow{2}{*}{$\mathfrak{O}$} & $1(4)$ & 1 & $K(2)^{2} \oplus K_{-1}(2)$ & $n$ \\
& $2(4)$ & 1 & $K(2) \oplus K_{\lambda}(4) \oplus K_{-4}(n)$ & $2 n$ \\
& $3(8)$ & 2 & $V_{1} \oplus K_{-2}(n)$ & $n$ \\
\hline \multirow{2}{*}{} & $1(4)$ & 1 & $K_{\lambda}(2)^{2} \oplus K_{-4}(n), \lambda=(-1)^{\left(m^{2}-1\right) / 4}$ & $2 n$ \\
& $2(4)$ & 1 & $K_{3}(4) \oplus K_{\lambda}(2) \oplus K_{-2}(n)$ & $n$ \\
& $3(8)$ & 1 & $K_{-4}(n)$ & $2 n$ \\
\hline
\end{tabular}

Here in all cases $f_{0}$ is any positive odd integer and

$$
\begin{aligned}
n & =m f_{0}^{2} \text { if } m \text { is odd and } \\
& =m^{\prime} f_{0} \text { if } m=2 m^{\prime} \text { with } m^{\prime} \text { odd. }
\end{aligned}
$$

For the notations $K_{\lambda}(l)$ and $V_{1}$ see 7.1,a). 1 (4) above means that $m \equiv 1$ (4) etc.

In the case $\mathbb{B}=\mathfrak{D}$ we have analogously the following:

Theorem 7.5. Let $T$ be a singular abelian surface. Let $(A, q)$ be the discriminant form of the transcendental lattice of $T$. Then a necessary and sufficient condition for $T$ to admit a special action of $D$ is that $(A, q)$ is isomorphic to one of the quadratic form groups listed in Table 17 below; in particular in this case $T$ is necessarily primitive. Further, the degree $\delta$ of any special pair $(T, G)$ with $G \cong \mathfrak{D}$ is given also in Table 17. The number of conjugacy classes in Aut $T$ of such groups $G$ is finite. 
Table 17

\begin{tabular}{|ll|c|}
\hline$(A, q)$ & & $\delta$ \\
\hline$K_{2}(3) \oplus K_{-2}(n)$ & $n \equiv 1,5(12)$ & $n$ \\
\hline$K_{-5}(6) \oplus K_{-1}(n)$ & $n \equiv 2,4(6)$ & $n / 2$ \\
\hline$K_{-6}(n)$ & $n \equiv 7(12)$ & $3 n$ \\
\hline $\begin{array}{l}K(2) \oplus K_{\lambda}\left(2^{k}\right) \oplus K_{-6 u}(n) \\
k>0, \lambda=1,5 \text { if } k>2 \\
=1 \text { if } k=1,2\end{array}$ & $n \equiv(-1)^{k}(8-3 \lambda)$ & (24) if $k \geqq 2$ \\
$\begin{array}{r}u=\left(3+(-1)^{k}\right) / 2 \\
\hline\end{array}$ & (12) if $k=1$ & $2^{k-1} 3 n$ \\
\end{tabular}

Remark 7.7. 1) As in the cases of $\mathscr{Q}$ and $\mathfrak{I}$ the existence of a special action of $\mathfrak{D}$, as well as the degree $\delta$ of a special pair $(T, G)$ with $G \cong \mathfrak{D}$, depends only on the genus to which $T$ belongs. Indeed, from the above table one can deduce the statements in terms of the invariant $\left(m, f_{0}, s\right)$ as in the first part of Theorem 7.2; but it becomes more complicated in this case so we have omitted it here.

2) Let $m$ be a square-free positive integer. Assume that $m=1,2$ or is a prime with $m \equiv 3,7$ (12). Then, similarly to Corollary 7.3 we see that any primitive singular abelian surface with invariant $(m, 1)$ admits a special action of $\mathfrak{D}$.

Remark 7.8. Each quadratic form group in Tables 16,17 is a discriminant form of a transcendental lattice of some singular abelian surface. This follows from $[15,1.10 .1]$ and the theorem of ShiodaMitani.

Example 7.9. The following is the list of all the singular abelian surfaces $T$ whose transcendental lattice $B_{T}$ has discriminant $D \leqq 12$, in its canonical representation as a product of elliptic curves (cf. 7. 1, g)). We also give i) all the possible non-cyclic groups \&f acting maximally and specially on $T$, ii) the degree $\delta$ of $(T, G)$ and iii) the number $c$ of conjugacy classes of such groups. 
Table 18

\begin{tabular}{|c|c|c|c|c|c|c|c|}
\hline$D$ & $T$ & $B_{T}$ & $\left(m, f_{0}, s\right)$ & $(A, q)$ & \& & $\delta$ & $c$ \\
\hline 3 & $E_{\rho} \times E_{\rho}$ & $\left(\begin{array}{ll}2 & 1 \\
1 & 2\end{array}\right)$ & $(3,1,1)$ & $K_{2}(3)$ & $\begin{array}{l}\mathfrak{I} \\
\mathfrak{D}\end{array}$ & $\begin{array}{l}6 \\
1\end{array}$ & $\begin{array}{l}1 \\
1\end{array}$ \\
\hline 4 & $E_{i}$ & $(2)^{2}$ & $(1,1$, & $K(2)^{2}$ & $\begin{array}{l}\mathfrak{Q} \\
\mathfrak{I} \\
\mathfrak{D}\end{array}$ & $\begin{array}{l}1 \\
2 \\
3\end{array}$ & $\begin{array}{l}1 \\
1 \\
1\end{array}$ \\
\hline 7 & $E_{\xi} \times E_{\xi}$ & $\left(\begin{array}{ll}2 & 1 \\
1 & 4\end{array}\right)$ & $(7,1,1)$ & $K_{-6}(7)$ & $\mathfrak{D}$ & 21 & 2 \\
\hline 8 & $E_{\sqrt{2} i} \times E_{\sqrt{2} i}$ & $(4) \oplus(2)$ & $(2,1,1)$ & $K(4) \oplus K(2)$ & $\stackrel{\mathscr{Q}}{\mathfrak{T}}$ & $\begin{array}{l}2 \\
1\end{array}$ & $\begin{array}{l}1 \\
1\end{array}$ \\
\hline 11 & $E_{\varphi} \times E_{\varphi}$ & $\left(\begin{array}{ll}2 & 1 \\
1 & 6\end{array}\right)$ & $(11,1,1)$ & $K_{6}(11)$ & $\mathfrak{I}$ & 22 & 1 \\
\hline 12 & $E_{\sqrt{3 i}} \times E_{\sqrt{3} i}$ & $(6) \oplus(2)$ & $(3,2,1)$ & $K_{-5}(6) \oplus K_{-1}(2)$ & D & 1 & 1 \\
\hline 12 & $E_{\rho} \times E_{\sqrt{3} i}$ & $\left(\begin{array}{ll}4 & 2 \\
2 & 4\end{array}\right)$ & $(3,1,2)$ & $V_{1} \oplus K_{-2}(3)$ & $\mathfrak{Q}$ & 3 & 1 \\
\hline
\end{tabular}

Here $\left.\xi=\omega_{7}, \varphi=\omega_{11}(\mathrm{cf} .7 .1, \mathrm{~b})\right)$. The table should be compared with Table 5 .

The above table can be checked as follows. First of all, from the relations (30), (34) and $D \leqq 12$, we get that the possible values of $\left(m, f_{0}, s\right)$ are exactly those in the above table. Let $h\left(K_{m}\right)=\# \mathscr{I}_{m, 1}$ be the class number of $K_{m}$. Then $h\left(K_{m}\right)=1$ if $m$ takes one of the values of the table (cf. Table 4 of [2]) and moreover $\# \mathscr{I}_{3,2}=1$ as follows from [2, p. 152]. It follows that for each possible vaule of $\left(m, f_{0}, s\right)$ there exists up to isomorphisms a unique binary positive even lattice $B$, which is exhibited in the above table. Moreover, one sees readily that each such $B$ admits an orientation-reversing automorphism so that the singular abelian surface $T$ whose transcendental lattice is isomorphic to $B$ is up to isomorphisms unique. $T$ is then obtained explicitly by (36). Finally, the values of $\mathbb{S}, \delta, c$ are obtained immediately from Theorems 7.2, 7.3, and 7.4, except the values of $c$ when $\mathbb{B}=\mathfrak{D}$, (for which we omit the proof here). 
7.3. We shall give the proofs of the above theorems.

Proof of Theorem 7.2. From Table 16 we first observe the following two facts: 1) A quadratic form group is isomorphic to one of those listed in Table 16 if and only if its invariant satisfies the conditions (i)-(iii) of the theorem, and 2) the quadratic form groups in the table have different invariants to one another. From these two facts it follows easily (cf. 7. 1, d)) that the theorem is a consequence of the following assertion: (A) Let $T$ be a singular abelian surface with the transcendental lattice $B_{T}$. Let $(A, q)$ be the discriminant form of $B_{T}$. Then there exists a finite subgroup $G$ of Aut $T$ such that $(T, G)$ is a maximal special pair if and only if $(A, q)$ is isomorphic to one of the quadratic form groups listed in Table 16. On the other hand, by Proposition 6.12, for (A) it suffices to show the next proposition.

Proposition 7.10. Let $\mathbb{S}=\mathfrak{Q}$ or $\mathfrak{I}(r e s p . \mathfrak{D})$. Let $B$ be a binary positive even lattice. Let $\left(A_{B}, q_{B}\right)$ be the discriminant form of $B$. Then there exists a primitive embedding $j: B \longrightarrow L_{\mathbb{\Theta}}(-1)$ if and only if $q_{B}$ is isomorphic to one of the quadratic form groups listed in Table 16 (resp. 17).

For the proof of Proposition 7.10 we need some preliminaries. Let $B$ be a binary, and $L$ a ternary, positive even lattices, respectively. Let $\left(A_{B}, q_{B}\right)$ and $\left(A_{L}, q_{L}\right)$ be the discriminant forms of $B$ and $L$, respectively. Then we consider the quintuples $(H, K, k, \gamma, \mu)$ consisting of finite subgroups $H$ of $A_{B}$, and $K$ of $A_{L}$, a positive integer $k$, and isomorphisms of quadratic form groups $\gamma:\left(H, q_{B \mid H}\right) \rightarrow\left(K, q_{L \mid K}\right)$ and $\mu: M \rightarrow K(2 k)$, where the quadratic form group $M$ is defined as follows. Let $\Gamma \leqq H \oplus K$ be the graph of $\gamma$ and $\Gamma^{\perp}$ the orthogonal complement of $\Gamma$ in $A_{B} \oplus A_{L}$ with respect to the quadratic form $\left(-q_{B}\right) \oplus q_{L}$. We denote by the same letter $\left(-q_{B}\right) \oplus q_{L}$ the quadratic form induced by it on $\Gamma^{\perp} / \Gamma$. Then we set

$$
M=\left(\Gamma^{\perp} / \Gamma,\left(-q_{B}\right) \oplus q_{L}\right) \text {. }
$$

Let $\mathscr{D}$ be the set of such quintuples. Let $E_{i}=\left(H_{i}, K_{i}, m_{i}, \gamma_{i}, \mu_{i}\right)$, $i=1,2$, be two quintuples in $\mathscr{D}$. Then we say that $E_{1}$ and $E_{2}$ are equivalent to each other if $H_{1}=H_{2}, k_{1}=k_{2}$, and if there exists an element $\xi \in \mathrm{O}\left(q_{L}\right)$ such that $\xi\left(K_{1}\right)=K_{2}, \gamma_{2}=\xi \gamma_{1}$, and $\mu_{1} \bar{\xi}= \pm \mu_{2}$, where 
$\bar{\xi}$ is the element of $\mathrm{O}(M)$ induced by $\xi$. The following is then a special case of Nikulin $[15,1.15 .1]$.

Proposition 7.11. Suppose that $L$ is uniquely determined by its discriminant form $\left(A_{L}, q_{L}\right)$ in the sense that if $L^{\prime}$ is another ternary positive even lattice with $\left(A_{L^{\prime}}, q_{L^{\prime}}\right) \cong\left(A_{L}, q_{L}\right)$, then $L$ is isomorphic to $L^{\prime}$. Then the set of equivalence classes of primitive embeddings of $B$ into $L$ is in natural bijective correspondences with the set of equivalence classes of quintuples in $\mathscr{D}$ as above. Moreover, if a primitive embedding $j: B \longrightarrow L$ corresponds to a quintuple $(H, K, k, \gamma, \mu)$ in $\mathscr{D}$, then the orthogonal complement of $j(B)$ in $L$ is isomorphic to the unary lattice $(2 k)$.

Let $H_{0}$ be any subgroup of $A_{L}$. Then we denote by $\mathscr{D}_{H_{0}}$ the set of quintuples $(H, K, k, \gamma, \mu)$ in $\mathscr{D}$ such that $H=H_{0}$. On the other hand, we consider the following conditions on $L$ (or on $A_{L}$ ): 1) For any subgroups $K_{1}, K_{2}$ of $A_{L}$ such that the resulting quadratic form groups $\left(K_{1}, q_{L \mid K_{1}}\right),\left(K_{2}, q_{L \mid K_{2}}\right)$ are isomorphic, there exists an element $\xi \in \mathrm{O}(L)$ such that $\xi\left(K_{1}\right)=K_{2}$, and 2) for any subgroup $K$ of $A_{L}$ the natural homomorphism $r_{K}: \mathrm{O}\left(q_{L}, K\right) \rightarrow \mathrm{O}\left(q_{L \mid K}\right)$ is surjective, where $\mathrm{O}\left(q_{L}, K\right)=\left\{\xi \in \mathrm{O}\left(q_{L}\right) ; \xi(K)=K\right\}$. Then the following is obvious from the definitions.

Lemma 7.12. Suppose that $L$ satisfies the conditions 1) and 2) above. Let $H_{0}$ be any subgroup of $A_{L}$. Fix any element $\left(H_{0}, K, k, \gamma, \mu\right)$ of $\mathscr{D}_{H_{0}}$. Then any element $\left(H^{\prime}, K^{\prime}, k^{\prime}, \gamma^{\prime}, \mu^{\prime}\right)$ of $\mathscr{D}_{H_{0}}$ is equivalent to a quintuple of the form $\left(H_{0}, K, k, \gamma, \mu^{\prime \prime}\right)$. Moreover, two quintuples ( $H_{0}$, $\left.K, k, \gamma, \mu_{i}\right), i=1,2$, are equivalent to each other if and only if there exists an element $\xi$ in the kernel of $r_{K}$ such that $\mu_{1} \bar{\xi}= \pm \mu_{2}$.

We are interested in the case $L=L_{\mathbb{\Theta}}(-1)$, where we recall $L_{0}=A_{1}^{3}$, $L_{\Upsilon}=A_{3}$ and $L_{\triangleright}=A_{1} \perp A_{2}$. First of all, we get the following lemma by direct computations (cf. also $[15,13.1]$ ).

Lemma 7.13. In the notations of 7.1 , a) the discriminant forms of $L_{\oplus}(-1)$ are given respectively $K(2)^{3}, K_{3}(4)$, and $K_{-5}(6) \cong K(2) \oplus K_{2}(3)$ according as $\mathbb{S}=\mathfrak{\Omega}, \mathfrak{I}$ and $\mathfrak{D}$.

From this we get immediately the following: 
Corollary 7.14. $L_{\Theta}(-1)$ satisfies the conditions 1) and 2) of Lemma 7. 12 .

Lemma 7.15. $L_{\oplus}(-1)$ is uniquely determined by its discriminant form in the sense of Proposition 7.11.

Proof. By Corollary 6.5 we know that the primitive embedding $L_{\oplus} \longleftrightarrow U^{3}$ (exists and) is unique. Hence the lemma follows from [15, 1.14.1].

Remark 7.16. A direct proof of Corollary 6.5 and Lemma 7.15 without using complex tori is as follows (cf。 Remark 6.6): First we consider the case $\mathbb{B}=\mathfrak{Q}$ so that $L_{\mathbb{\Theta}}(-1)=A_{1}^{3}$ and $q_{L}=K(2)^{3}$. Then for any ternary positive even lattice $C$ with $q_{C} \cong q_{L}, C(1 / 2)$ is a ternaty positive unimodular lattice. Hence $G(1 / 2) \cong(1)^{3}$ (cf。 [16, 106:13]) so that $C \cong A_{1}^{3}$. Hence Lemma 7.15 is proved in this case. Since the natural homomorphism $\mathrm{O}\left(A_{1}^{3}\right) \rightarrow \mathrm{O}\left(K(2)^{3}\right)$ is surjective, Corollary 6.5 follows from $[15,1.14 .1]$. For $L_{\varkappa}$ and $L_{\diamond}$ Corollary 6.5 is an easy consequence of $[15,1.14 .6]$ and the fact that $q_{L_{x}} \cong q_{D_{5}}$ and $q_{L_{\triangleright}} \cong q_{A_{5}}$, where $D_{5}$ and $A_{5}$ are considered negative root lattices (cf. $[15,13.1]$ ). As above this also gives a proof of Lemma 7. 15.

Proof of Proposition 7.10. We treat only the case $\mathbb{G}=\mathfrak{Q}$, other cases being essentially the same. Let $L=L_{\mathbb{B}}(-1)$ in Proposition 7.11. By Lemma 7.15 $L$ certainly satisfies the condition of that proposition. By Lemma 7.13 we can identify the discriminant form $\left(A_{L}, q_{L}\right)$ with $K(2)^{3}$. Now let $E=(H, K, k, \gamma, \mu)$ be any quintuple in $\mathscr{D}$ considered up to equivalence. Since $K \cong \subseteq_{2}^{3}$ and $H$ is isomorphic to $K, H$ is contained in the 2-torsion part $A_{2}$ of $A_{B}$, and hence $\Gamma \leqq A_{2} \oplus A_{L}$; therefore if $\Gamma_{\frac{1}{2}}^{\perp}$ is the orthogonal complement of $\Gamma$ in $A_{2} \oplus A_{L}$ we have $\Gamma^{\perp}=\Gamma_{2}^{\perp} \oplus A_{2}^{\prime}$, where $A_{2}^{\prime}=\bigoplus_{p \neq 2} A_{p}$ with $A_{p}$ the $p$-torsion part of $A_{B}$. Hence

$$
\Gamma^{\perp} / \Gamma \cong \Gamma_{2}^{\perp} / \Gamma \oplus A_{2}^{\prime} .
$$

Then, since $\Gamma^{\perp} / \Gamma \cong \mathfrak{夭}_{2 k}, \Gamma_{2}^{\perp} / \Gamma$ (and $\left.A_{2}^{\prime}\right)$ must be cyclic. Let $K \cong \mathfrak{C}_{2}^{b}$, $0 \leqq b \leqq 3$. Then $b \neq 3$ as rank $B=2$. Moreover, if $K^{\perp}$ is the orthogonal complement of $K$ in $A_{L}$, we have the natural inclusion $K^{\perp} \oplus 0 \longrightarrow \Gamma \frac{\perp}{2} / \Gamma$. 
Since $K^{\perp} \cong \overleftarrow{F}_{2}^{3-b}$ and $\Gamma_{2}^{\perp} / \Gamma$ is cyclic, it follows that $b \geqq 2$; thus $b=2$. Then, after a transformation by a suitable element of $\mathrm{O}\left(q_{L}\right)$ if necessary we can assume that $K$ coincides with one of the following quadratic form subgroups a) $\left\langle e_{1}, e_{2}\right\rangle \cong K(2)^{2}$, b) $\left\langle e_{1}, e_{2}+e_{3}\right\rangle \cong K(2) \oplus$ $K_{2}(2)$, and c) $\left\langle e_{1}+e_{2}, e_{1}+e_{3}\right\rangle \cong V_{1}$, where $\left\{e_{1}, e_{2}, e_{3}\right\}$ is the standard basis of $A_{L}=K(2)^{3}$.

Correspondingly, $\left(A_{2}, q_{2}\right)$ must contain a quadratic form subgroup which is isomorphic to: a) $K(2)^{2}$, b) $K(2) \oplus K_{2}(2)$, c) $V_{1}$, respectively. Since $\left(A_{2}, q_{2}\right)$ is isomorphic to one of the quadratic form groups $K_{\lambda}\left(2^{k}\right) \oplus K_{\theta}\left(2^{l}\right), \quad 0 \leqq k \leqq l$, and $U_{k}, V_{k}, k \geqq 1$ (cf.7. 1, a)), we easily conclude that 1) $\left(A_{2}, q_{2}\right)$ must be isomorphic to: a) $K(2)^{2}$, b) $K(2)$ $\oplus K_{\lambda}(4), \lambda= \pm 1, \pm 5$, and c) $V_{1}$, respectively, where in b) the natural inclusion $K_{2}(2) \longrightarrow K_{\lambda}(4)$ induces the subgroup $K(2) \oplus K_{2}(2)$. It then follows that $M_{2}:=\left(\Gamma_{\frac{1}{2}} / \Gamma,\left(-q_{B}\right) \oplus q_{L \mid \Gamma_{2}} / \Gamma\right)$ is isomorphic to
a) $K(2)$,
b) $K_{2-\lambda}(4)$, and
c) $K_{-1}(2)$

in respective cases. (Use the fact that by Corollary 7.14 and Lemma 7.12 we may take $\gamma$ to be any isomorphism of $H$ and $K$ to caluculate $M_{2}$. ) Write these groups as $K_{\eta}\left(2^{l}\right)$ in general ; for instance $\eta=l=1$ in case a). Let $n$ be the order of $A_{2}^{\prime}$ so that we have

$$
k=2^{l-1} n \text {. }
$$

Then we have $M \cong K(2 k) \cong K\left(2^{l} n\right) \cong K_{n}\left(2^{l}\right) \oplus K_{2^{l}}(n)$; by comparing this with (37) we get that $M_{2} \cong K_{n}\left(2^{l}\right)$ and $\left(A_{2}^{\prime},-q_{2}^{\prime}\right) \cong K_{2^{l}}(n)$. Therefore $\left(A_{B}, q_{B}\right) \cong\left(A_{2}, q_{2}\right) \oplus K_{-2^{l}}(n)$ with $K_{n}\left(2^{l}\right) \cong K_{\eta}\left(2^{l}\right)$, while the last condition is equivalent to: $n \equiv \eta$ (8) (resp. (4)) if $l \geqq 2$ (resp. $=1$ ). From this we get that $\left(A_{B}, q_{B}\right)$ must be isomorphic to one of the following groups: a) $K(2)^{2} \oplus K_{-2}(n)$ with $n \equiv 1 \quad(4)$, b) $K(2) \oplus K_{2}(2) \oplus K_{-1}(n)$ with $n \equiv 2-\lambda$ (8) and c) $V_{1} \oplus K_{-2}(n)$ with $n \equiv 3$ (4).

Finally, the fact that $\left(A_{B}, q_{B}\right)$ is a discriminant form of the lattice $B$ imposes additional restrictions on the possible structures of $\left(A_{B}, q_{B}\right)$. In fact, by $[15,1.10 .1]$ (the condition 4) there in our case) we see that in case c) we must have $n \equiv 3$ (8). (Note that in the cases $\mathfrak{I}$ and $\mathscr{D}$ this restriction turns out to be much stricter.) Hence $\left(A_{B}, q_{B}\right)$ must be isomorphic to one of the quadratic form groups in Table 16 for $\mathbb{S}=\mathfrak{Q}$.

Conversely, if $\left(A_{B}, q_{B}\right)$ is isomorphic to one of the quadratic form groups in Table 16, then from the above arguments one sees readily 
that we can find at least one quintuple $(H, K, k, \gamma, \mu)$ in $\mathscr{D}$. Hence by Proposition 7.11 there exists a primitive embedding $j: B \longrightarrow L$ 。

Proof of Theorem 7.4. Again we consider only the case $\mathbb{G}=\mathfrak{Q}$, the case $\mathbb{S}=\mathfrak{I}$ being similar. Let $(T, G)$ be a maximal special pair with $G \cong \mathscr{Q}$ and with $T$ a singular abelian surface. Let $B_{T} \longrightarrow L_{\Theta}(-1)$ be the primitive embedding of the transcendental lattice $B_{T}$ of $T$ into $L_{\mathbb{\Theta}}(-1)$ determined by $(T, G)$ (cf. Proposition 6.12). Let ( $H, K, k$, $\gamma, \mu)$ be the quintuple in $\mathscr{D}$ corresponding to this embedding (cf. Proposition 7.11). Then, since the $G$-invariant Neron-Severi lattice $N_{T}^{G}$ is given by $N_{T}^{G} \cong B_{T}^{\perp} \cong L_{\Theta}$ by Proposition 5 . 9 , we have $N_{T}^{G} \cong(2 k)$ by Proposition 7. 11. Hence $\delta=k$. We then have $\delta=2^{l-1} n$ as in (39). It then follows readily that $\delta$ depends only on the structure of $\left(A_{B}, q_{B}\right)$ as in Table 16 (in fact on the order of $A_{B}$ ) and is as listed in Table 16. This shows 1 ).

Next we show 2). $T$ determines the binary positive even lattice with fixed orientation $B_{T}$, and hence, the finite group $A_{B}$. Moreover, as the proof of the previous proposition shows, even the subgroup $H$ of $A_{B}$ is uniquely determined once $A_{B}$ is fixed, hence depending only on $T$, but not on the choice of the maximal special subgroup $G \cong$ Aut $T$. Hence, by Proposition 7.11 the set of isomorphism classes of maximal special pairs $\left(T, G_{1}\right)$ with $G_{1} \cong G$ is in natural bijective correspondence with the set of equivalence classes of the quintuples in $\mathscr{D}_{H}$. Further, by Lemma 7.12 and Corollary 7.14 we see that the latter in turn is identified with the set $\mathscr{M}$ of isomorphisms $\mu: M \rightarrow K(2 \delta)$ with $\mu^{\prime} s$ considered modulo the following equivalence; $\mu \sim \mu^{\prime}$ if and only if $\mu \bar{\xi}= \pm \mu^{\prime}$ for some element $\xi \in \mathrm{O}\left(K(2)^{3}\right) \cong \widetilde{S}_{3}$ which induces the identity on $K \cong K(2)^{3}$ (cf。 Lemma 7.12)。

Let $M_{2}$ be the 2-torsion part of $M$ and $M_{2}^{\prime}$ be its orthogonal complement. Then clearly any $\bar{\xi}$ as above induces the identity on $M_{2}^{\prime}$, and if $M_{2} \cong K(2)$ or $K_{-1}$ (2) (cf. (38)), also on $M_{2}$. Hence, under the latter assumption $\mathscr{M}$ is in bijective correspondence with the set $\mathrm{O}(K(2 \delta)) /\langle-1\rangle$, which is isomorphic as a group to $\mathfrak{夭}_{2}^{t-1}, \delta$ being odd (cf. Table 16). In case $M_{2} \cong K_{2-\lambda}(4)$ simple computation shows that any element of $\mathrm{O}\left(M_{2}\right) \cong\langle-1\rangle$ is induced by some $\bar{\xi}$ as above. Thus $\mathscr{M}$ is in bijective correspondence with the set $O\left(M_{2}^{\prime}\right) /\langle-1\rangle$. Since $M_{2}^{\prime}$ is cyclic of (odd) order $\delta / 2$, the latter is isomorphic as a 
group again to $\mathfrak{\mho}_{2}^{t-1}$ (cf. 7.1, a)).

Proof of Theorem 7.5. The first assertion follows from Proposition 7. 10. The values of $\delta$ can be computed essentially the same way as in the case of $\mathfrak{Q}$; the detail will be omitted. The finiteness of $G$ follows from Proposition 7.11 and the finiteness of the set $\mathscr{D}$.

7.4. a) By using Theorems 7.2 and 7.4 we can give another proof for Proposition 3.15. Indeed, generalization to certain nonprincipal polarizations is also possible. For instance we have the following:

Proposition 7.17. Let $\mathbb{S}=\mathfrak{Q}$ (resp. $\mathfrak{I}$, resp. D). Let $(T, G)$ be a maximal special pair with $G \cong ® s$. Then $T$ admits a $G$-invariant polarization of degree $\delta \leqq 3$ if and only if $T$ is isomorphic to one of the singular abelian surfaces in the table below. Conversely, given a singular abelian surface $T$ in the table there exists up to isomorphisms a unique such pair $(T, G)$. Moreover, in this case the $G$-invariant polarization is unique and is invariant also by the normalizer of $G$ in Aut $T$.

\begin{tabular}{|c|c|c|c|c|c|c|}
\hline (S) & $\delta$ & $T$ & $\delta$ & $T$ & $\delta$ & $T$ \\
\hline $\mathfrak{O}$ & \multirow{4}{*}{1} & $E_{i} \times E_{i}$ & \multirow{4}{*}{2} & $E_{\sqrt{2} i} \times E_{\sqrt{2} i}$ & \multirow{4}{*}{3} & $E_{\rho} \times E_{\sqrt{3} i}$ \\
\hline $\mathfrak{T}$ & & $E_{\sqrt{2} i} \times E_{\sqrt{2} i}$ & & $E_{i} \times E_{i}$ & & $E_{\sqrt{6} i} \times E_{\sqrt{6} i}$ \\
\hline \multirow{2}{*}{ () } & & $E_{\rho} \times E_{\rho}$ & & \multirow{2}{*}{$E_{(\sqrt{6} \bar{i}) / 2} \times E_{\sqrt{6} i}$} & & \multirow{2}{*}{$E_{i} \times E_{i}$} \\
\hline & & $E_{\sqrt{3} i} \times E_{\sqrt{3} i}$ & & & & \\
\hline
\end{tabular}

Proof. We show the first and the second assertions. Since the case $\delta=1$ is just Proposition 3.15, we only consider the cases $\delta=2$ or 3. From Tables 16 and 17 we pick up the cases for which $\delta=2$ or 3. As a result the possible values of invariants $\left(m, f_{0}, s\right)$ turn out to be as follows: Case $\delta=2:(2,1,1)$ if $\mathbb{S}=\mathfrak{O},(1,1,1)$ if $\mathbb{S}=\mathfrak{I}$, and $(6,1,1)$ if $\mathbb{S}=\mathfrak{D}$. Case $\delta=3:(3,1,2)$ if $\mathbb{S}=\mathfrak{O},(6,1,1)$ if $\mathbb{S}=\mathfrak{T}$, and $(1,1,1)$ if $\mathbb{S}=\mathfrak{D}$. Then in each case the corresponding singular abelian surface is unique and is isomorphic to one of the singular 
abelian surfaces in Table 18, and hence the assertion is proved, except for the cases $\delta=2, \mathbb{S}=\mathfrak{D}$ and $\delta=3, \mathbb{S}=\mathfrak{T}$. In these cases the discriminant forms are isomorphic respectively to $K_{-5}(6) \oplus K_{-1}(4)$ and $K_{3}(4) \oplus K(2) \oplus K_{-2}(3)$, which are indeed isomorphic to the discriminant form of the lattices $(4) \oplus(6)$ and $(2) \oplus(12)$, respectively. Since $h\left(K_{6}\right)=2$, these exhaust the positive even lattices with discriminant 24 up to isomorphisms. Then by the same argument as in the proof of Example 7.9 we see that in each of these cases $T$ is unique and is isomorphic to the corresponding singular abelian surface in the table. The last assertion is proved in the same way as the corresponding one in Proposition 3.15.

b) The groups $\mathfrak{Q}, \mathfrak{I}$ and $\mathfrak{D}$ are characterized as those noncommutative finite groups which can be realized as a subgroup of $\mathrm{SL}\left(2, K_{m}\right)$ for some square-free positive integer $m$; the subgroup is then unique up to conjugacy. More precisely, by Proposition 5. 13, the following holds true:

(S) is realized as a subgroup of $\mathrm{SL}_{2}\left(K_{m}\right)$ if and only if $m \neq \equiv-1$ (resp. $m \not \equiv-1(3)$ ) provided that $\mathbb{S}=\mathfrak{Q}$ or $\mathfrak{I}$ (resp. $\mathfrak{D})$.

We consider then the problem as to when (S) can be realized as a subgroup of $\mathrm{SL}_{2}\left(\mathfrak{o}_{m}\right)$, where $\mathfrak{o}_{m}$ is the maximal order of $K_{m}$ and then would also like to calculate the number of conjugacy classes. Since $\mathrm{SL}_{2}\left(\mathfrak{O}_{m}\right)$ is identified with the subgroup of Aut $E_{\omega} \times E_{\omega}, \omega=\omega_{m}$, of all the special automorphisms, the answer is in a sense already contained in Theorems 7.2 and 7.4. We shall state this only in the case $\mathbb{S}=\mathfrak{O}$ or $\mathfrak{T}$. (In the case $\mathbb{S}=\mathfrak{D}$, the statement becomes less simple.)

Proposition 7.18. Write $m=2^{\alpha} p_{1} \ldots p_{s}$, where $\alpha=0$ or 1 and $p_{i}$ are distinct odd primes. Then the necessary and sufficient condition that $\mathfrak{D}$ (resp. $\mathfrak{I})$ is realized as a subgroup of $\mathrm{SL}_{2}\left(\mathfrak{o}_{m}\right)$ is that $p_{i} \equiv 1$ (4) (resp. $p_{i} \equiv 1$ or 3 (8)) for any i. Moreover, if $m$ satisfies the above condition, then the number of conjugacy classes under $\mathrm{GL}_{2}\left(\mathfrak{D}_{m}\right)$ of the maximal finite subgroups of $\mathrm{SL}_{2}\left(\mathfrak{D}_{m}\right)$ which are isomorphic to $\mathfrak{Q}$ (resp. I) equals $2^{s-1}$. (When $m=2^{\alpha}$ we set $s=1$.)

Proof. As before we consider only the case $\mathbb{S}=\mathfrak{O}$. Fixing an embedding $K_{m} \longrightarrow \mathbb{C}$ we obtain an elliptic curve $E_{m}:=\mathbb{C} / \mathfrak{o}_{m}$. Set $T_{m}=E_{m} \times E_{m}$. Then Aut $T_{m} \cong \mathrm{GL}_{2}\left(\mathfrak{D}_{m}\right)$. With respect to this isomorphism 
we can naturally identify the set of conjugacy classes under $\mathrm{GL}_{2}\left(\mathfrak{D}_{m}\right)$ of maximal finite subgroups of $\mathrm{SL}_{2}\left(\mathrm{D}_{m}\right)$ which is isomorphic to $G$ with the set of conjugacy classes of maximal special subgroups of Aut $T_{m}$. By (36) we see that the transcendental lattice $B_{m}$ of $T_{m}$ is isomorphic to $(2) \oplus(2 m)$ if $\mathrm{m} \equiv 1$ or $2(4)$, and to $\left(\begin{array}{ll}2 & 1 \\ 1 & 2 k\end{array}\right)$ if $m \equiv 3$ (4), where $k=(m+1) / 4 \geqq 1$. Then the corresponding discriminant forms are given respectively by $K(2)^{2} \oplus K_{2}(m)$ if $m \equiv 1$ (4), $K(2) \oplus K_{l}(4) \oplus$ $K_{4}(l), l=m / 2$, if $m \equiv 2(4)$, and $K_{2 k}(m)$ if $m \equiv 3$ (4). Comparing this with Table 16 we see that $T$ admits a maximal special action of $\mathfrak{Q}$ if and only if either of the following is true; a) $m \equiv 1$ (4) and $K_{-2}(m) \cong K_{2}(m)$, and b) $m \equiv 2$ (4) and $K_{-4}(l) \cong K_{4}(l)$. These conditions are reduced to the condition that -1 is a quadratic residue modulo $m$ and modulo $l$ in cases a) and b) respectively, which in turn is equivalent to $p_{i} \equiv 1$ (4) for any $i$. This proves the first part of the proposition. Since in this case $\delta=m$, the second assertion follows from Theorem 7.4.

Remark 7.19. Starting from Proposition 6.12 and proceeding in the same way as in 7.3 we can also obtain the condition for a singular abelian surface $T$ to admit a special $\mathfrak{c}_{k}$-action, $k=4$ or 6 , in terms of the discriminant form $\left(A_{T}, q_{T}\right)$ of $B_{T}$. For instance, in case of $\mathfrak{F}_{6}$, the condition is: a) for any prime $p$ with $p \equiv-1$ or 5 (12), the $p$-torsion part $A_{p}$ of $A_{T}$ is cyclic and b) either the 2-torsion part $A_{2}$ of $A_{T}$ is cyclic or $\left(A_{2}, q_{2}\right)$ admits $K_{ \pm 1}(2)$ as a direct summand. In particular, if $A_{T}$ is cyclic, then $T$ always admits a special $\mathfrak{F}_{6}$-action.

\section{References}

[1] Atiyah, M. F., Some examples of complex manifolds, Bonn. Math. Sch., 6 (1958).

[2] Borevič, Z. I., and Šafarevič, I. R., Number theory, Academic Press, 1966.

[ 3 ] Calabi, E., Isometric families of Kähler structures, In: The Chern Symposium 1979, Berlin-Heidelberg New York: Springer, 1980, 23-40.

[4] Dickson, L. E., Algebren und ihre Zahlentheorie, Zürich, 1927.

[5] Enriques, F., and Severi, F., Memoire sur les surfaces hyperelliptiques, Acta Math., 32 (1909), 283-392, 33 (1910), 321-403.

[6] Fujiki, A., On primitively symplectic compact Kähler V-manifolds of dimension four, In: Cassification theory of algebraic and analytic manifolds, Progress in Math., 39, Birkhäuser (1983), 71-250.

[7] On endomorphism rings of complex tori, in preparation.

[8] Gottschling, E., Über die Fixpunkte der Siegelschen Modulgruppe, Math. Ann., 143 
(1961), 111-149.

[ 9 ] Gottschling, E., Über die Fixpunktuntergruppen der Siegelschen Modulgruppe, Math. Ann., 143 (1961), 399-430.

[10] Hasse, H., Number theory, Grund. der Math. Wiss., 229, Springer, 1980.

[11] Humphreys, J. E., Introduction to Lie algebras and representation theory, GTM, BerlinHeidelberg-New York, Springer, 1972.

[12] Katsura, T., Generalized Kummer surfaces and their unirationality in characteristic p, preprint.

[13] Namikawa, Y., and Ueno, $K$., On fibers in families of curves of genus 2, I. Number theory, algebraic geometry, and commutative algebra, Kinokuniya, Tokyo, 1973, 297371.

[14] Nikulin, V. V., Finite automorphism groups of Kähler K3 surfaces, Trans. Moscow Math. Soc., 2 (1980), 71-135.

[15] - Integral symmetric bilinear forms and some of their applications, Math. USSR Izvestija, 14 (1980), 103-167.

[16] O’Mehara, O. T., Introduction to quadratic forms, Grund. der Math. Wiss., Springer, 1971.

[17] Reiner, I., Maximal orders, Academic Press, London, 1975.

[18] Serre, J.-P., A course in arithmetic, GTM, 7, Springer, 1973.

[19] Shapiro, R. A., Pseudo-hermitian symmetric spaces, Comment. Math. Helv., 46 (1971), 529-548.

[20] Shimura, G., On analytic families of polarized abelian varieties and automorphic functions, Ann. of Math., 78 (1963), 149-193.

[21] Introduction to the arithmetic theory of automorphic functions, Publ.of Math. Soc. Japan, Iwanami Publ. and Princeton Univ. Press. 1971.

[22] Shioda, T., The period map of abelian surfaces, J. Fac. Sci. Univ. Tokyo, Sec. IA, 25 (1978), 47-59.

[23] Shioda, T., and Mitani, N., Singular abelian surfaces and binary quadratic forms, Lecture Notes in Math., 412, Springer, 1974, 259-287.

[24] Tokunaga, S., and Yoshida, M., Complex crystallographic groups I, J. Math. Soc. Japan, 34 (1982), 581-605.

[25] Ueno, K., On fiber spaces of normally polarized abelian varieties of dimension 2, I-II., J. Fac. Sci. Univ. of Tokyo, 188 (1971), 37-95, 189 (1972), 163-199.

[26] Vignera, M.-F., Arithmétique des algèbres de quaternions, Lecture Notes in Math., 800, Springer, 1980.

[27] Yoshihara, H., Structure of complex tori with the automorphisms of maximal degree, Tsukuba J. Math., 4 (1980), 303-311.

[28] Zarhin, Y. G., Hodge groups of K3 surfaces, J. Reine u. Angew. Math. 391 (1983), $193-220$ 
